\title{
Probing effective field theory operators in the associated production of top quarks with a $Z$ boson in multilepton final states at $\sqrt{s}=13 \mathrm{TeV}$
}

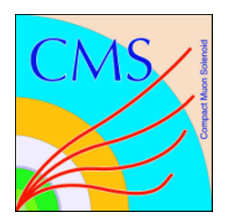

\section{The CMS collaboration}

E-mail: cms-publication-committee-chair@cern.ch

ABSTRACT: A search for new top quark interactions is performed within the framework of an effective field theory using the associated production of either one or two top quarks with a $\mathrm{Z}$ boson in multilepton final states. The data sample corresponds to an integrated luminosity of $138 \mathrm{fb}^{-1}$ of proton-proton collisions at $\sqrt{s}=13 \mathrm{TeV}$ collected by the CMS experiment at the LHC. Five dimension-six operators modifying the electroweak interactions of the top quark are considered. Novel machine-learning techniques are used to enhance the sensitivity to effects arising from these operators. Distributions used for the signal extraction are parameterized in terms of Wilson coefficients describing the interaction strengths of the operators. All five Wilson coefficients are simultaneously fit to data and $95 \%$ confidence level intervals are computed. All results are consistent with the SM expectations.

KEYwords: Hadron-Hadron scattering (experiments), Top physics

ArXiv EPRINT: 2107.13896

We dedicate this publication to our friend and colleague Nicolas Tonon who passed away unexpectedly while this paper was in print. This work would not have been possible without Nicolas' major contributions. 


\section{Contents}

1 Introduction 1

2 The CMS detector 4

3 Data sample and Monte Carlo simulations 5

3.1 Simulation of the signal samples 6

3.2 Parameterization of the event weights 6

4 Event reconstruction $\quad 7$

5 Event selection and categorization $\quad 9$

6 Background estimation 11

7 Multivariate analysis $\quad 12$

$\begin{array}{ll}\text { 7.1 Discrimination between SM processes } & 13\end{array}$

7.2 Discrimination between the SM and EFT scenarios 13

8 Systematic uncertainties $\quad 16$

8.1 Experimental uncertainties 16

$\begin{array}{lll}8.2 & \text { Theoretical uncertainties } & 17\end{array}$

$\begin{array}{lll}8.3 & \text { Background uncertainties } & 18\end{array}$

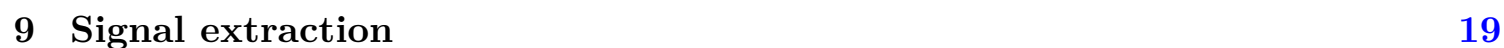

$\begin{array}{ll}10 \text { Results } & 20\end{array}$

$\begin{array}{ll}11 \text { Summary } & 25\end{array}$

$\begin{array}{ll}\text { The CMS collaboration } & 34\end{array}$

\section{Introduction}

The standard model (SM) of particle physics is supported by a vast number of measurements from experiments covering a broad energy range up to the $\mathrm{TeV}$ scale. However, the SM falls short of explaining several observed phenomena, such as neutrino oscillations [1] and the baryon asymmetry in our universe [2], and cannot accommodate the existence of dark matter and dark energy [3]. More generally, there are many indications that the SM corresponds to a low-energy approximation of a more fundamental theory beyond the standard model (BSM). Several BSM models explain these phenomena by postulating the 
existence of new particles or mechanisms. However, these hypothetical new particles may be too massive to be directly accessible at the CERN LHC.

Even in the absence of any direct observation of a new particle, new phenomena may manifest themselves indirectly through corrections at the quantum-loop level, thus leading to observable deviations in well-established processes. Such deviations can be interpreted in a coherent and model-independent manner using the approach of effective field theory (EFT) $[4,5]$. An EFT corresponds to an approximation at low energy of an underlying theory characterized by an energy scale $\Lambda$ that is well above the typical energies accessible at colliders. New, effective interactions between the SM fields are introduced by extending the SM Lagrangian with higher-order operators. The interaction strength of an operator of dimension $d$ is characterized by a dimensionless Wilson coefficient (WC) and is proportional to $1 / \Lambda^{d-4}$. This factor suppresses the contribution from higher-order operators, implying that effects of new interactions can be approximated with a finite set of WCs associated with lower-order operators. Since operators with $d=5$ or 7 violate lepton and/or baryon number conservation [6], we restrict our analysis to dimension-six operators. The effective Lagrangian can then be written as

$$
\mathcal{L}_{\text {eff }}=\mathcal{L}_{\mathrm{SM}}+\sum_{i} \frac{c_{i}}{\Lambda^{2}} \mathcal{O}_{i}
$$

where $\mathcal{L}_{\mathrm{SM}}$ is the SM Lagrangian, $\mathcal{O}_{i}$ are dimension-six operators, and $c_{i}$ are the corresponding WCs that can be constrained from experimental data.

The large top quark mass $m_{\mathrm{t}}=172.44 \pm 0.49 \mathrm{GeV}[7]$ corresponds to a Yukawa coupling to the Higgs boson close to unity. This suggests that the top quark may play a special role within the SM, and that its precise characterization may shed light on the electroweak symmetry breaking mechanism [8-10]. The high integrated luminosity and center-of-mass energy at the LHC make it possible to study rare top quark processes, such as the associated production of top quarks with a $\mathrm{Z}$ boson, where top quarks are either produced in pairs ( $\mathrm{t} \overline{\mathrm{Z}}$ ), or singly in the $\mathrm{tZq}$ and $\mathrm{tWZ}$ channels. Representative tree-level Feynman diagrams are shown for the three signal processes in figure 1 . Whereas the $t \bar{t} Z$ and $t Z q$ processes have comparable inclusive cross sections of about $800 \mathrm{fb}$ and were already observed by both the ATLAS and CMS Collaborations [11-14], the tWZ process has a much smaller cross section and has not been observed yet. These three processes are of major interest because they probe the coupling of the top quark to the $\mathrm{Z}$ boson at tree level. Numerous BSM extensions predict sizable modifications to this coupling [15-17], which is among the least constrained by the available data in the top quark sector.

The focus of the present analysis is the study of the electroweak interactions of the top quark, and in particular the $t \bar{t} Z$ interaction. Consequently, we only consider EFT operators involving third-generation quarks and gauge bosons, which interfere with the $\mathrm{SM}$ production of the $\mathrm{t} \overline{\mathrm{tZ}}$ or $\mathrm{tZq}$ signal. This means that these operators must affect either process - or both - at order $1 / \Lambda^{2}$. We restrict our study to CP-conserving effects, and thus we ignore the imaginary components of complex WCs. Finally, the $\mathcal{O}_{\mathrm{tG}}$ operator related to the chromomagnetic dipole moment of the top quark is ignored, since it can be probed with much better sensitivity in $t \bar{t}$ events [18]. As a result, we focus on a subset of five 


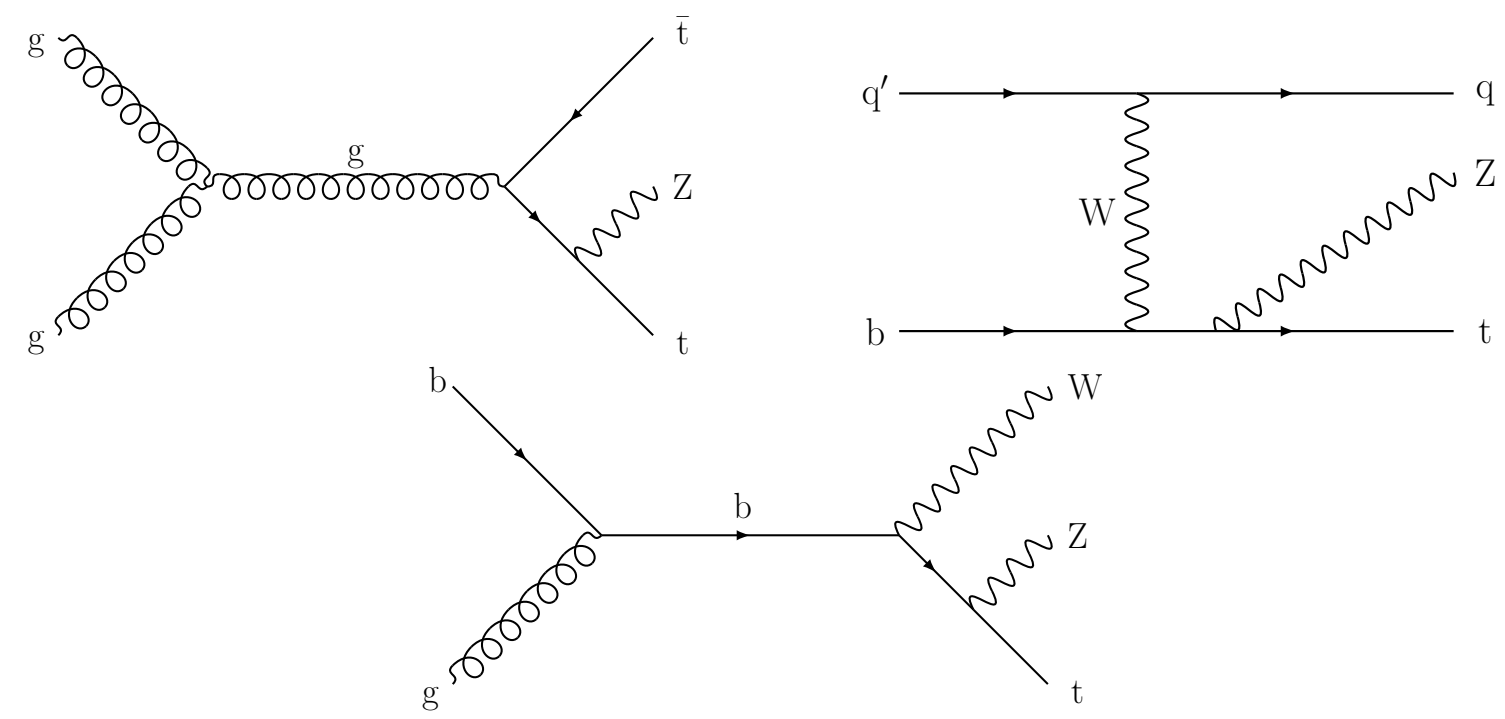

Figure 1. Representative Feynman diagrams at tree level for $t \bar{t} Z$ (upper left), $t Z q$ (upper right), and tWZ (lower) production.

operators, namely: $\mathcal{O}_{\mathrm{tZ}}, \mathcal{O}_{\mathrm{tW}}, \mathcal{O}_{\varphi \mathrm{Q}}^{3}, \mathcal{O}_{\varphi \mathrm{Q}}^{-}$, and $\mathcal{O}_{\varphi \mathrm{t}}[19]$. The $\mathcal{O}_{\mathrm{tZ}}$ and $\mathcal{O}_{\mathrm{tW}}$ operators induce electroweak dipole moments of the top quark, $\mathcal{O}_{\varphi \mathrm{Q}}^{3}$ is the left-handed $\mathrm{SU}(2)$ triplet current operator, and the $\mathcal{O}_{\varphi \mathrm{Q}}^{-}$and $\mathcal{O}_{\varphi \mathrm{t}}$ neutral-current operators modify the interactions of the $\mathrm{Z}$ boson with left- and right-handed top quarks, respectively. Comprehensive descriptions of their effects on top quark interactions are given in refs. [20,21].

A key aspect of this study is the use of novel multivariate analysis (MVA) techniques based on machine learning to enhance the sensitivity to new phenomena arising from the EFT operators. Since new operators usually affect the distributions of multiple observables, MVA techniques that exploit correlations in high-dimensional data are well suited for EFT measurements. We train machine-learning algorithms for two purposes. Firstly, a multiclass classifier is trained to distinguish between different SM processes, and is used to define subregions enriched either in signal or background events. Secondly, binary classifiers are trained to separate events generated according to the SM from events generated with nonzero WC values for one or more EFT operators. They are used to construct powerful discriminating observables that are ultimately fit to data to compute two confidence intervals for each WC, one keeping the other WCs fixed to zero and the other treating all five WCs as free parameters. The core ideas for these binary classifiers appeared recently in the literature and since then have garnered increased attention, notably because they were shown to outperform traditional approaches based on single observables in several case studies at the generator level [22-24]. This motivates the application of this technique for the first time in an LHC analysis involving the interference between EFT operators and the SM amplitude.

The binary classifiers are trained with simulated samples whose event weights are parameterized as functions of the five WCs of interest. These samples are passed through a full detector simulation, and are used to search for new interactions without making any simplifying assumption regarding the parton shower and detector response. A previous 
CMS analysis in the top quark sector employed this approach to parameterize new interactions directly at the detector level in the context of an EFT [25]. It used data collected in 2017 and targeted multilepton final states. That analysis set constraints on 16 WCs simultaneously by performing counting experiments in various event categories, which were defined based on the jet and lepton multiplicities, the presence of a $\mathrm{Z}$ boson candidate, and the sum of the lepton charges.

The data sample of proton-proton (pp) collisions at $\sqrt{s}=13 \mathrm{TeV}$ used in this paper was collected by the CMS experiment during Run 2 of the LHC (2016-2018) and corresponds to an integrated luminosity of $138 \mathrm{fb}^{-1}$, of which 36.3 [26], 41.5 [27], and 59.7 [28] $\mathrm{fb}^{-1}$ were recorded in 2016, 2017, and 2018, respectively. We target events in which the decays of the top quark and Z boson lead to final states with three or four leptons, either electrons or muons; this also includes a small contribution from leptonic tau lepton decays. Unless stated otherwise and throughout this paper, the term lepton refers exclusively to electrons and muons, generically denoted by $\ell$. These multilepton final states offer high trigger efficiencies, and a more favorable signal-to-background ratio compared with hadronic channels.

The paper is organized as follows. We provide a brief overview of the CMS detector in section 2. Section 3 describes the data and simulated event samples, as well as the parameterization of the weights for simulated events. Section 4 details the object and event reconstructions, while the event selection and categorization are presented in section 5 . The estimation of backgrounds is presented in section 6 . The MVA is described in section 7 , the systematic uncertainties affecting the measurements in section 8 , and the signal extraction procedure in section 9 . We discuss the results in section 10 , and conclude with a summary of the paper in section 11. Tabulated results are provided in HEPData [29].

\section{The CMS detector}

The central feature of the CMS apparatus is a superconducting solenoid of $6 \mathrm{~m}$ internal diameter, providing a magnetic field of $3.8 \mathrm{~T}$. Within the solenoid volume are a silicon pixel and strip tracker, a lead tungstate crystal electromagnetic calorimeter (ECAL), and a brass and scintillator hadron calorimeter, each composed of a barrel and two endcap sections. Forward calorimeters extend the pseudorapidity $(\eta)$ coverage provided by the barrel and endcap detectors. Muons are detected in gas-ionization chambers embedded in the steel flux-return yoke outside the solenoid.

Events of interest are selected using a two-tiered trigger system. The first level (L1), composed of custom hardware processors, uses information from the calorimeters and muon detectors to select events at a rate of around $100 \mathrm{kHz}$ within a fixed latency of about $4 \mu \mathrm{s}$ [30]. The second level, known as the high-level trigger, consists of a farm of processors running a version of the full event reconstruction software optimized for fast processing, and reduces the event rate to around $1 \mathrm{kHz}$ before data storage [31]. A more detailed description of the CMS detector, together with a definition of the coordinate system and of the relevant kinematic variables, can be found in ref. [32]. 


\section{Data sample and Monte Carlo simulations}

The data sample was recorded using a combination of single-, double-, and triple-lepton trigger algorithms, whose thresholds on the transverse momentum $p_{\mathrm{T}}$ with respect to the beam axis vary between data-taking periods depending on the instantaneous luminosity. For instance, the minimum $p_{\mathrm{T}}$ threshold of the single-electron (-muon) triggers ranges between $25-35(22-27) \mathrm{GeV}$. For events selected in this analysis, the combined trigger efficiency is nearly $100 \%$ both in data and simulation.

Event samples produced via Monte Carlo (MC) simulation are used to estimate the contributions of signal processes and most background processes, as well as to train machinelearning algorithms. The signal samples are generated at leading order (LO) in perturbative quantum chromodynamics (QCD) and incorporate EFT effects, whereas background samples do not include EFT effects and are generated at next-to-LO (NLO) whenever possible. Additional signal samples generated at NLO without EFT effects are used for validation purposes, and to train a classification algorithm aiming to separate different SM processes, as described in section 7 . The latter samples will be referred to explicitly as "SM signal samples". The mass of the top quark is set to $m_{\mathrm{t}}=172.5 \mathrm{GeV}$ in simulation.

The SM signal samples for the $\mathrm{tZq}$ and $\mathrm{t} \overline{\mathrm{t} Z}$ processes, as well as the samples for several background processes ( $\mathrm{WZ}, \mathrm{t} \overline{\mathrm{t}} \mathrm{W}, \mathrm{t} \overline{\mathrm{t}} \mathrm{t} \overline{\mathrm{t}}$, multiboson production, and $\mathrm{V} \gamma$, where $\mathrm{V}$ denotes either a $\mathrm{W}$ or $\mathrm{Z}$ boson), are generated at NLO using MADGRAPH5_aMC@NLO 2.4.2 [33]. The SM tWZ sample and other background samples are generated at LO

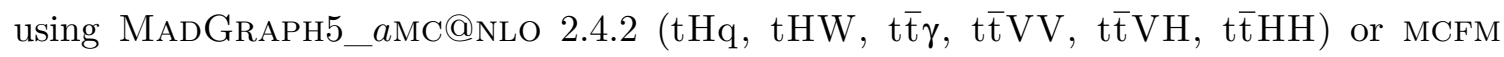

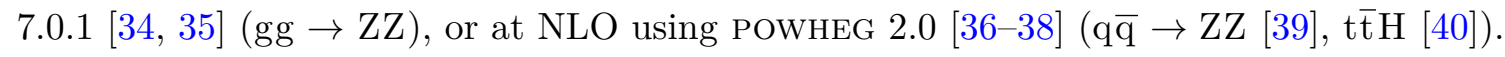
The SM tZq sample is generated in the four-flavor scheme, in which only up, down, strange, and charm quarks are considered as sea quarks of the proton, whereas the SM tWZ sample is generated in the five-flavor scheme, in which bottom quarks are considered as sea quarks of the proton as well and may appear in the initial state of pp scattering processes [41].

The NNPDF3.1 [42] set of parton distribution functions (PDFs) is used to simulate signal samples for all three years, and background samples for 2017-2018, and the NNPDF3.0 [43] set of PDFs is used to generate background samples for 2016. The parton showering, hadronization, and underlying event are modeled using PYTHIA 8.2 [44] with the tune CP5 [45] for the 2017-2018 samples, as well as for the 2016 samples for the signal, t $\bar{t} \mathrm{~W}$, $\mathrm{t} \overline{\mathrm{t}} \mathrm{H}, \mathrm{t} \overline{\mathrm{t}} \gamma, \mathrm{Z} \gamma$, and $\mathrm{t} \overline{\mathrm{t}} \overline{\mathrm{t}}$ processes, whereas the tunes CUETP8M1 or CUETP8M2T4 [46, 47] are used to simulate other background samples for 2016. The matching of matrix elements (MEs) to the parton shower (PS) is done using the FxFx [48] merging scheme for NLO samples, and the MLM scheme [49] for LO samples.

The presence of simultaneous pp collisions in the same or nearby bunch crossings, referred to as pileup, is modeled by superimposing inelastic pp interactions simulated using PYTHIA 8.2 on all generated events. Generated events are passed through a detailed simulation of the CMS detector based on GEANT4 [50], and are reconstructed with the same version of the CMS event reconstruction software used for data. 


\begin{tabular}{|lll|}
\hline Operator & WC & Mapping to Warsaw-basis coefficients \\
\hline $\mathcal{O}_{\mathrm{tZ}}$ & $c_{\mathrm{tZ}}$ & $\operatorname{Re}\left\{-s_{\mathrm{W}} c_{\mathrm{uB}}^{(33)}+c_{\mathrm{W}} c_{\mathrm{uW}}^{(33)}\right\}$ \\
$\mathcal{O}_{\mathrm{tW}}$ & $c_{\mathrm{tW}}$ & $\operatorname{Re}\left\{c_{\mathrm{uW}}^{(33)}\right\}$ \\
$\mathcal{O}_{\varphi \mathrm{Q}}^{3}$ & $c_{\varphi \mathrm{Q}}^{3}$ & $c_{\varphi \mathrm{q}}^{3(33)}$ \\
$\mathcal{O}_{\varphi \mathrm{Q}}^{-}$ & $c_{\varphi \mathrm{Q}}^{-}$ & $c_{\varphi \mathrm{q}}^{1(33)}-c_{\varphi \mathrm{q}}^{3(33)}$ \\
$\mathcal{O}_{\varphi \mathrm{t}}$ & $c_{\varphi \mathrm{t}}$ & $c_{\varphi \mathrm{u}}^{(33)}$ \\
\hline
\end{tabular}

Table 1. List of dimension-six EFT operators considered in this analysis and their corresponding WCs. The linear combinations of WCs to which they correspond in the Warsaw basis are indicated. The abbreviations $s_{\mathrm{W}}$ and $c_{\mathrm{W}}$ denote the sine and cosine of the weak mixing angle, respectively. The definitions of the relevant Warsaw-basis operators can be found in ref. [19].

Residual differences between data and simulation are corrected by modifying the weights of generated events, or by varying relevant simulated quantities. Such differences are observed in: the pileup distribution; the reconstruction and identification efficiencies for electrons and muons; the jet energy scale and resolution; the efficiency to identify jets originating from the hadronization of bottom quarks and the corresponding misidentification rates for light $(\mathrm{u}, \mathrm{d}, \mathrm{s}, \mathrm{c})$-quark and gluon jets; and the resolution in missing transverse momentum.

\subsection{Simulation of the signal samples}

The signal samples including EFT effects are generated at LO using MAdGraPh5_aMC@NLO 2.6.5 and the NNPDF3.1 PDF set. The decays of top quarks and $\mathrm{W}$ bosons are simulated with the MADSPIN program [51].

We generate the signal events following a similar approach to that outlined in ref. [19]. The EFT model used in the present analysis focuses on dimension-six operators that give rise to interactions involving at least one top quark. The degrees of freedom of this model are defined as linear combinations of Warsaw-basis operator coefficients [52], and the mapping between both bases is given in table 1. Since this model only allows for tree-level generation, the tEZ sample is generated with an extra parton in the final state to improve its accuracy. The MLM merging scheme is used to match the MEs to the PS. However, the tZq sample does not include an extra final-state parton because this matching procedure cannot be performed correctly for single top quark processes in the $t$ channel (due to the presence of a final-state light quark in the MEs), and neither does the tWZ sample to avoid

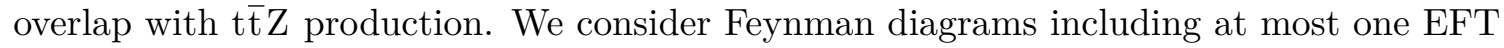
vertex, in which the top quark is produced.

\subsection{Parameterization of the event weights}

To interpret potential deviations in signal production in terms of new interactions, we parameterize the weights of the simulated signal events with the five WCs of interest. Within 
an EFT framework, a given ME may be decomposed into its SM and EFT components as

$$
\mathcal{M}=\mathcal{M}_{\mathrm{SM}}+\mathcal{M}_{\mathrm{EFT}}=\mathcal{M}_{\mathrm{SM}}+\sum_{i} \frac{c_{i}}{\Lambda^{2}} \mathcal{M}_{i}
$$

where $\mathcal{M}_{\mathrm{SM}}$ is the $\mathrm{SM} \mathrm{ME}, \mathcal{M}_{i}$ are the MEs associated with the new operators, and $c_{i}$ are WCs. A production cross section, either inclusive or differential, is proportional to the square of the total ME and can thus be written as a polynomial of second order in the WCs. The weight for a given event can then be parameterized with a five-dimensional (5D) quadratic function of the WCs as

$$
w\left(\frac{\vec{c}}{\Lambda^{2}}\right)=s_{0}+\sum_{j} s_{1, j} \frac{c_{j}}{\Lambda^{2}}+\sum_{j} s_{2, j}\left(\frac{c_{j}}{\Lambda^{2}}\right)^{2}+\sum_{j} \sum_{k} s_{3, j k} \frac{c_{j}}{\Lambda^{2}} \frac{c_{k}}{\Lambda^{2}},
$$

where the sums run over the five WCs, $\vec{c}$ is the set of WC values, and the $s_{0}, s_{1}, s_{2}, s_{3}$ coefficients are associated with: the SM amplitude; the interference between SM and EFT amplitudes; EFT amplitudes; and the interference among EFT amplitudes, respectively. Although individual coefficients may be negative or null, the sum of all components always yields a physical distribution.

Following the procedure adopted in ref. [25], this analysis makes extensive use of the possibility offered by the MADGRAPH5_aMC@NLO event generator to assign multiple weights to an event, which represent the infinitesimal contributions from this event to the total cross section at different points in the EFT phase space. Each simulated event is associated with weights corresponding to different WC values, which are used to build an overdetermined system of equations to solve for all the per-event coefficients -21 in total — of eq. (3.2). By summing the quadratic functions of all simulated events for a given signal process, one can then evaluate its differential cross section as a function of any quantity, at any point in the 5D EFT phase space. The parameterized event yields of the signal samples are normalized such that they equal their SM NLO theoretical predictions $[53,54]$ when all WCs are set to zero.

\section{Event reconstruction}

The particle-flow algorithm [55] combines information from all subdetectors to reconstruct individual particles in an event, and to identify them either as electrons, muons, photons, charged hadrons, or neutral hadrons.

The candidate vertex with the largest value of summed physics-object $p_{\mathrm{T}}^{2}$ is taken to be the primary pp interaction vertex. The physics objects in this context are the jets, clustered using the jet-finding algorithm $[56,57]$ with the tracks assigned to candidate vertices as inputs, and the negative vector $p_{\mathrm{T}}$ sum of those jets. Reconstructed lepton candidates are required to have track parameters compatible with the primary vertex as origin.

Electron candidates are reconstructed within the range $|\eta|<2.5$ by combining the energy measurement in the ECAL with the momentum measurement in the tracker [58]. They are required to satisfy $p_{\mathrm{T}}>7 \mathrm{GeV}$, and their identification relies upon an MVA algorithm trained with observables related to the ECAL and tracker measurements. Electrons 
originating from photon conversion are efficiently removed by requiring that candidate tracks have at most one missing hit in the innermost tracker layers.

Muon candidates are reconstructed within the range $|\eta|<2.4$ as tracks in the tracker consistent with either a track or several hits in the muon system, and associated with calorimeter deposits compatible with the muon hypothesis [59]. They are required to satisfy $p_{\mathrm{T}}>5 \mathrm{GeV}$, as well as a set of quality criteria designed to reject hadrons punching through the calorimeters and muons produced by in-flight decays of kaons and pions.

Electron and muon candidates satisfying the aforementioned selection criteria are referred to as "loose leptons". Additional selection criteria are applied to select genuine "prompt" leptons produced in decays of $\mathrm{W}$ and $\mathrm{Z}$ bosons and leptonic $\tau$ decays, while rejecting "nonprompt" leptons (NPLs) mainly originating from b hadron decays, hadron misidentification, and the conversion of photons not produced in the hard scattering interaction. Background events containing at least one NPL, arising mostly from $t \bar{t}+$ jets and $\mathrm{Z} / \gamma^{\star}$ production, will be referred to as "NPL background" throughout this paper. The rejection of NPLs is significantly improved by the use of MVA discriminants based on boosted decision trees [60]. They take as input several observables related to the lepton and to the jet activity in its vicinity. Electron and muon candidates satisfying a selection on the MVA discriminants are referred to as "tight leptons".

Jet candidates are reconstructed offline from energy deposits in the calorimeter towers, and clustered using the anti- $k_{\mathrm{T}}$ algorithm $[56,57]$ with a distance parameter of 0.4 . They are required to satisfy $p_{\mathrm{T}}>25 \mathrm{GeV}$ and $|\eta|<5$, and must not overlap with any loose lepton within $\Delta R=\sqrt{(\Delta \eta)^{2}+(\Delta \phi)^{2}}<0.4$, where $\phi$ is the azimuthal angle. The contribution from pileup to jet momentum is mitigated by excluding charged hadrons associated with pileup vertices from the clustering. The energy of reconstructed jets is corrected for residual pileup effects, and calibrated as a function of jet $p_{\mathrm{T}}$ and $\eta[61,62]$. We apply the more stringent requirement $p_{\mathrm{T}}>60 \mathrm{GeV}$ to jets reconstructed within the range $2.7<|\eta|<3$ to suppress calorimeter noise. Jets passing these selection criteria are categorized into central and forward jets, the former satisfying the condition $|\eta|<2.4$, and the latter $2.4<|\eta|<5$. The presence of a high- $p_{\mathrm{T}}$ forward jet in the event, referred to as a recoiling jet, is a characteristic feature of $\mathrm{tZq}$ production that is used in the MVA to isolate the contribution from this process. The phase space extension due to the inclusion of forward jets increases the acceptance of the $\mathrm{tZq}$ signal by about $25 \%$ in the trileptonic signal region defined in section 5 .

Jets arising from bottom quarks are identified (b tagged) with the DEEPJET deep neural network algorithm [63, 64], within the ranges $|\eta|<2.4$ in 2016 and $|\eta|<2.5$ in 2017-2018 because of the Phase-1 upgrade of the CMS pixel detector [65]. Jets passing the medium working point of the algorithm are referred to as "b jets". For central jets with $p_{\mathrm{T}}>$ $30 \mathrm{GeV}$, this corresponds to a selection efficiency of about $75 \%$ for jets arising from bottom quarks, and to a misidentification rate for light-quark and gluon jets of about $1 \%$ [66].

The missing transverse momentum vector $\vec{p}_{\mathrm{T}}^{\text {miss }}$ is computed as the negative vector $p_{\mathrm{T}}$ sum of all the particle-flow candidates in an event, and its magnitude is denoted by $p_{\mathrm{T}}^{\text {miss }}[67]$. The $\vec{p}_{\mathrm{T}}^{\text {miss }}$ is modified to account for corrections to the energy scale of the reconstructed jets in the event. 
After the final-state particles have been identified and selected, we combine their information to reconstruct unstable particles that are expected in the topologies of the signal processes (see figure 1). These higher-level objects are used for event categorization and to construct powerful observables provided as input to the MVA algorithms presented in section 7 .

The $\mathrm{Z}$ boson candidates are reconstructed from pairs of opposite-sign same-flavor leptons having invariant masses within $15 \mathrm{GeV}$ of the true mass $m_{\mathrm{Z}}$ of the $\mathrm{Z}$ boson. While this analysis considers events with either three or four leptons, we do not reconstruct tetraleptonic events further since their kinematic information is not exploited in the signal extraction procedure described in section 9. In trileptonic events with multiple $\mathrm{Z}$ boson candidates, only the candidate whose mass is closest to $m_{\mathrm{Z}}$ is considered. The vector $\vec{p}_{\mathrm{T}}^{\text {miss }}$ is associated with the undetected neutrino arising from the leptonic top quark decay. The longitudinal neutrino momentum is obtained by applying the $\mathrm{W}$ boson mass constraint and solving an analytical equation. The leptonically decaying top quark is then reconstructed using the four-momentum of the neutrino, the remaining selected lepton, and one $\mathrm{b}$ jet. In case two neutrino solutions or multiple $\mathrm{b}$ jets are reconstructed, the combination resulting in a top quark mass closest to $m_{\mathrm{t}}$ is chosen. The $\mathrm{W}$ boson transverse mass $m_{\mathrm{T}}^{\mathrm{W}}$ is reconstructed from the $\mathrm{W} \rightarrow \ell \nu$ decay products and constitutes a powerful observable to discriminate background processes such as $\mathrm{ZZ}, \mathrm{Z} / \gamma^{\star}$, and $\mathrm{Z} \gamma$. To improve the selection of the $\mathrm{tZq}$ signal, we identify the remaining jet with the largest $p_{\mathrm{T}}$ in the event as the recoiling jet, with a veto on $b$ jets. In the rare case that an event contains only $b$ jets, this veto is removed. The lepton asymmetry $\mathrm{q}_{\ell}|\eta(\ell)|$ is defined as the product of the charge and absolute pseudorapidity of the lepton originating from the top quark decay, and provides additional discrimination power for the $\mathrm{tZq}$ signal.

\section{Event selection and categorization}

This analysis targets the associated production of top quarks with a $\mathrm{Z}$ boson, in events where the $\mathrm{Z}$ boson and at least one top quark decay leptonically. The corresponding experimental signatures are characterized by the presence of: three or four prompt leptons; high $p_{\mathrm{T}}^{\text {miss }}$ due to the neutrino(s) from $\mathrm{W}$ boson decay(s); at least one $\mathrm{b}$ jet from top quark decay; and possibly additional light-quark jets, either produced in the decays of top quarks or $\mathrm{W}$ bosons, or recoiling against the single top quark.

The signal region (SR) targeting trileptonic signal events drives the sensitivity of the

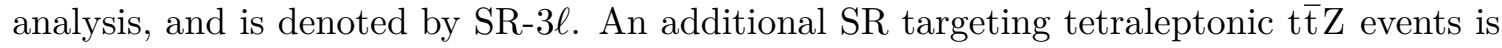

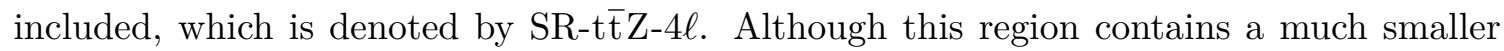
amount of data compared with the SR-3 $\ell$, it is pure in $\mathrm{t} \overline{\mathrm{t} Z}$ events and thus provides additional sensitivity to operators impacting the cross section of this signal.

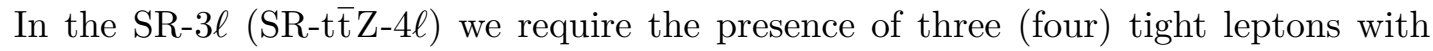
$p_{\mathrm{T}}>25,15,10$ (and 10) GeV. To reject lepton pairs originating from low-mass hadron decays, which are not well modeled by the MC simulation, events containing a pair of loose leptons having an invariant mass below $12 \mathrm{GeV}$ are discarded. Since this analysis focuses on the $t \bar{t} \mathrm{Z}$ interaction, we require the presence of exactly one $\mathrm{Z}$ boson candidate in events 

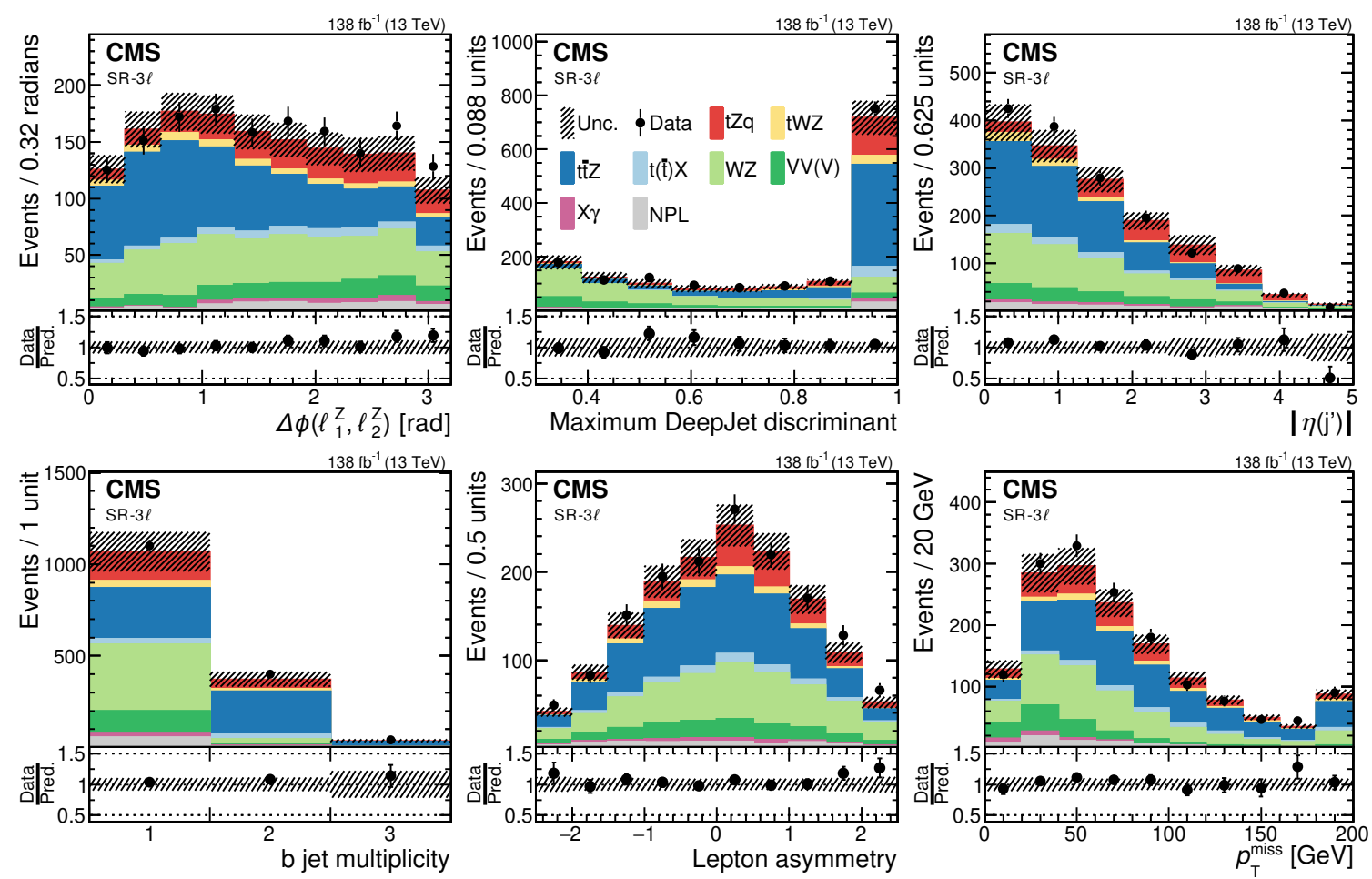

Figure 2. Pre-fit data-to-simulation comparisons for several observables in the SR-3 $\ell$. From left to right and upper to lower, the distributions correspond to: the relative azimuthal angle $\Delta \phi$ between the two leptons from the $\mathrm{Z}$ boson decay; the maximum DEEPJET discriminant among all selected jets; the absolute pseudorapidity of the recoiling jet; the $\mathrm{b}$ jet multiplicity; the lepton asymmetry; and $p_{\mathrm{T}}^{\text {miss }}$. The lower panels display the ratios of the observed event yields to their predicted values. The NPL background is modeled with the procedure based on control samples in data described in section 6 . The hatched band represents the total uncertainty in the prediction. Underflows and overflows are included in the first and last bins, respectively.

entering the SRs. They are also required to contain at least two jets, and at least one b jet. Additionally, we require the sum of the lepton charges to be zero in events entering the

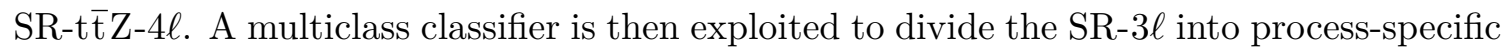
subregions, as explained in section 7. Figure 2 shows data-to-simulation comparisons in the SR-3 $\ell$ for several observables that are most relevant for this classification, before performing a fit to data as described in section 9 (pre-fit).

The main irreducible background contribution in the SR- $3 \ell$ arises from the production of two vector bosons, predominantly via the $\mathrm{WZ} \rightarrow 3 \ell$ process. The $\mathrm{ZZ} \rightarrow 4 \ell$ process also contributes, in cases where one lepton fails the lepton identification or is out of detector acceptance. These events contain on average fewer jets (b tagged or not) compared with signal processes, and are efficiently suppressed by the requirements on the jet and $\mathrm{b}$ jet multiplicities. Background processes with three vector bosons (WWW, WWZ, WZZ, and ZZZ) are considered as well and represent a minor contribution; together with the $\mathrm{ZZ}$ process, they are denoted by $\mathrm{VV}(\mathrm{V})$. Other processes with top quarks contributing as irreducible backgrounds are $t \bar{t} \mathrm{H}, \mathrm{t} \overline{\mathrm{t}} \mathrm{W}, \mathrm{tHW}, \mathrm{tHq}, \mathrm{t} \overline{\mathrm{t}} \mathrm{VV}, \mathrm{t} \overline{\mathrm{t}} \mathrm{VH}, \mathrm{t} \overline{\mathrm{t}} \mathrm{HH}$, and $\mathrm{t} \overline{\mathrm{t}} \mathrm{t} \overline{\mathrm{t}}$; they are denoted by 


\begin{tabular}{|c|c|c|c|c|}
\hline Selection requirement & 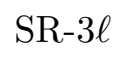 & 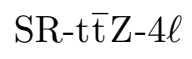 & WZ CR & ZZ CR \\
\hline Lepton multiplicity & $=3$ & $=4$ & $=3$ & $=4$ \\
\hline$m_{3 \ell}-m_{\mathrm{Z}}$ & - & - & $>15 \mathrm{GeV}$ & - \\
\hline $\mathrm{Z}$ boson candidates multiplicity & $=1$ & $=1$ & $=1$ & $=2$ \\
\hline Jet multiplicity & $\geq 2$ & $\geq 2$ & - & - \\
\hline b jet multiplicity & $\geq 1$ & $\geq 1$ & $=0$ & - \\
\hline$p_{\mathrm{T}}^{\mathrm{miss}}$ & - & - & $>50 \mathrm{GeV}$ & - \\
\hline
\end{tabular}

Table 2. Summary of the main selection requirements applied in each signal or control region. A dash indicates that the selection requirement is not applied.

$\mathrm{t}(\overline{\mathrm{t}}) \mathrm{X}$. The contribution from processes including a photon produced in the hard scattering interaction is denoted by $\mathrm{X} \gamma$, and is dominated by $\mathrm{Z} \gamma$ and $t \overline{\mathrm{t}} \gamma$ events yielding two prompt leptons plus a photon that undergoes an asymmetric conversion. The NPL background is modeled with the procedure based on control samples in data described in section 6 .

Two control regions (CRs) are enriched in the main background processes. They are included in the signal extraction procedure to constrain the uncertainties in the cross sections of these backgrounds from the data and are defined as follows:

- A CR enriched in WZ events ("WZ CR") is defined similarly to the SR-3l, except that events containing one or more $\mathrm{b}$ jet(s) are rejected and no requirement on the minimal jet multiplicity is applied. The purity in WZ events is increased by requiring $p_{\mathrm{T}}^{\text {miss }}>50 \mathrm{GeV}$, and the invariant mass of the trileptonic system $m_{3 \ell}$ to be larger than $m_{\mathrm{Z}}+15 \mathrm{GeV}$.

- A CR enriched in ZZ events ("ZZ CR") is defined by requiring the presence of exactly four tight leptons that are compatible with two $\mathrm{Z}$ boson candidates. No requirements on the jet and b jet multiplicities are applied.

All regions are orthogonal by construction, and the leptons selected in the CRs must satisfy the same $p_{\mathrm{T}}$ thresholds as used in the SRs. A summary of the main selection requirements applied in each region is provided in table 2 .

\section{Background estimation}

Irreducible backgrounds containing genuine prompt leptons are reliably estimated from MC simulations and are constrained in dedicated CRs. The X $\gamma$ background is strongly suppressed by the lepton identification criteria and its remaining contribution is estimated from MC simulations. However, the NPL background is known to be much more challenging to model. Thus, its contribution is estimated using the data-based misidentification probability method [60]. This method relies on the selection of samples of events satisfying the same selection criteria as defined in section 5 for the different regions, except that the lepton identification requirements are relaxed. We refer to these samples as the application regions (ARs) of the misidentification probability method. 
Estimates of the NPL background contribution to the different regions are obtained by applying suitably-chosen weights to the corresponding AR events. These weights quantify the probability for NPLs passing the relaxed identification requirements to be misidentified as tight leptons. They are measured in a data sample enriched in events composed uniquely of jets produced via the strong interaction, referred to as multijet events, in which an NPL originating from one of the jets is reconstructed. These data are collected with single muon (electron) triggers, some of which require the presence of an additional jet with $p_{\mathrm{T}}>40$ (30) GeV. Events are selected if they contain exactly one lepton passing the relaxed identification criteria, plus at least one jet separated by $\Delta R>0.7$ from the lepton. The weights are measured separately for electrons and muons, and parameterized with the $p_{\mathrm{T}}$ and $\eta$ of the lepton candidate. The selected events are divided into "pass" and "fail" samples, depending on whether the lepton passes or fails the tight identification criteria, respectively.

The contribution from multijet events dominates the fail sample and is estimated directly from the data, after subtracting the small contamination from prompt lepton events (mostly from $\mathrm{W}+$ jets, $\mathrm{Z} / \gamma^{\star}$, diboson, and $\mathrm{t} \overline{\mathrm{t}}+$ jets production) based on MC simulations. It is then fit to the data in the pass sample, along with the contribution from prompt lepton events as estimated from the simulations, to determine the number of multijet events in the pass sample. The misidentification probability in a given category is computed as $w=N_{\text {pass }} /\left(N_{\text {pass }}+N_{\text {fail }}\right)$, where $N_{\text {pass }}$ and $N_{\text {fail }}$ are the number of multijet events in the pass and fail samples, respectively. To prevent potential double counting in the NPL background estimation, all tight leptons selected in signal and control regions are matched to their generator-level equivalents in the simulation, and simulated events containing at least one lepton qualifying as nonprompt are discarded. Further details on the procedure for the estimation of the NPL background can be found in ref. [60].

\section{Multivariate analysis}

This analysis makes extensive use of MVA techniques based on neural networks (NNs) to enhance the sensitivity to new phenomena arising from the EFT operators of interest. Firstly, a multiclass classifier is trained to separate the contributions from the main SM processes in the SR-3l. It is used to define three subregions enriched in the $t Z q, t \overline{t Z}$, and background processes. Secondly, binary classifiers are trained to separate events generated according to the SM from events generated with nonzero WC values. The responses of these NNs represent optimal observables that are used for the signal extraction in the SR-3 3 subregions.

All trainings are performed with MC simulations in the SR-3l, using the TensoRFLOw [68] package with the KeRAS interface [69] and ADAM optimizer [70]. The training phase aims to reduce the cross-entropy loss function [71], and the NN weights are updated using batch gradient descent. Potential overtraining is mitigated using dropout [72], L2 regularization [73], and early stopping in case the minimized function has reached a stable minimum. The number of equidistant bins $N_{\text {bins }}$ and the range of each NN output distribution are adjusted such that the total expected event yield is above one in all bins. The bins are numbered from 1 to $N_{\text {bins. }}$. 


\subsection{Discrimination between SM processes}

The SR-3l selection criteria are designed to retain a large proportion of signal events while rejecting most background events. However, basic selection criteria cannot isolate efficiently the rare signals from the overwhelming background processes that yield similar final states. Moreover, each signal process probes the WCs in unique ways, and the shapes of their kinematic distributions may be impacted differently in the presence of new interactions. To take advantage of this complementarity, it is thus desirable to separate the signal processes from one another.

To this end, we train a multiclass NN classifier, denoted hereafter by "NN-SM". It is tasked with isolating the $\mathrm{tZq}$ and $\mathrm{t} \overline{\mathrm{t}} \mathrm{Z}$ signals from the major $\mathrm{WZ}, \mathrm{t}(\overline{\mathrm{t}}) \mathrm{X}$, and $\mathrm{VV}(\mathrm{V})$ backgrounds. The tWZ signal is not targeted explicitly because it has a comparatively small event yield and is kinematically close to the tẼ $\mathrm{Z}$ process. This classifier is trained using the SM signal samples, which represent our best available SM predictions at NLO accuracy for these processes.

The NN-SM includes three hidden layers, each with 100 rectified linear units [74], and has three output nodes labeled "tZq", "t€ZZ", and "Others". The response of the NN-SM is used to divide the SR- $3 \ell$ into three subregions, each enriched in a particular class of events, which we label accordingly "SR-tZq", "SR-t€Z", and "SR-Others". The softmax activation function is used for all output nodes. Their activation values may be interpreted as the probability for a given event to be either $\mathrm{tZq}$ signal, $\mathrm{t} \overline{\mathrm{tZ}}$ signal, or background. Since the softmax operation normalizes the output vector to unit magnitude by construction, we assign a given event entering the SR- $3 \ell$ to either of the orthogonal subregions based on the output node that is most activated by this event. The set of 33 variables used as input to the NN-SM comprises 12 "high-level" variables that are designed to improve the separation between the different process classes. The three-momenta $\left(p_{\mathrm{T}}, \eta, \phi\right)$ of the three leading leptons and up to three leading jets in the events, as well as the b tagging discriminants for these jets, are also included and improve the classification performance. All the input variables are listed in table 3 . Their distributions and correlations with other variables are verified to be properly modeled by the simulations.

The pre-fit data-to-simulation comparisons for the distributions of the three output nodes of the NN-SM are shown in figure 3. For each distribution, only the events that have their maximum value in the corresponding output node are included.

\subsection{Discrimination between the SM and EFT scenarios}

In a second step, we leverage the EFT parameterization of the signal event weights to train NNs tasked with separating events generated either according to the SM, or according to EFT scenarios in which at least one WC is nonzero. These classifiers are denoted by "NNEFTs" hereafter. They are used to design observables with optimal sensitivity to new effects arising from the targeted operators. These techniques are based on ideas developed in the context of likelihood-free inference techniques, which are described extensively in ref. [22].

Classification algorithms were already used in previous analyses adopting an EFT framework. For instance, ref. [75] takes advantage of a multiclass classifier to separate 


\begin{tabular}{|c|c|c|c|c|c|c|c|c|c|}
\hline Variable & 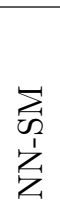 & 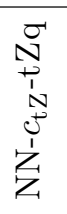 & 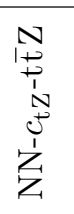 & 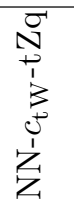 & 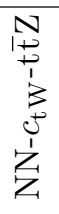 & 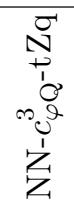 & 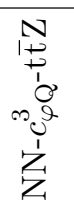 & 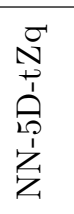 & 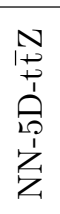 \\
\hline$p_{\mathrm{T}}^{\mathrm{Z}}$ & - & $\checkmark$ & $\checkmark$ & $\checkmark$ & $\checkmark$ & $\checkmark$ & $\checkmark$ & $\checkmark$ & $\checkmark$ \\
\hline$\eta(Z)$ & $\checkmark$ & $\checkmark$ & $\checkmark$ & - & - & $\checkmark$ & - & - & $\checkmark$ \\
\hline$\Delta \phi\left(\ell_{1}^{Z} \ell_{2}^{Z}\right)$ & $\checkmark$ & $\checkmark$ & $\checkmark$ & $\checkmark$ & $\checkmark$ & $\checkmark$ & $\checkmark$ & $\checkmark$ & $\checkmark$ \\
\hline$p_{\mathrm{T}}(\mathrm{t})$ & $\checkmark$ & $\checkmark$ & $\checkmark$ & - & $\checkmark$ & $\checkmark$ & - & $\checkmark$ & $\checkmark$ \\
\hline$\eta(\mathrm{t})$ & - & $\checkmark$ & $\checkmark$ & $\checkmark$ & $\checkmark$ & $\checkmark$ & - & - & $\checkmark$ \\
\hline$m(\mathrm{t}, \mathrm{Z})$ & - & - & - & - & - & - & - & - & - \\
\hline$\left|\eta\left(j^{\prime}\right)\right|$ & $\checkmark$ & - & - & - & - & - & - & $\checkmark$ & - \\
\hline$p_{\mathrm{T}}\left(j^{\prime}\right)$ & $\checkmark$ & $\checkmark$ & - & $\checkmark$ & - & - & - & - & - \\
\hline$\Delta R\left(b, \ell_{\mathrm{t}}\right)$ & - & $\checkmark$ & - & $\checkmark$ & - & - & - & - & - \\
\hline$\Delta R\left(j^{\prime}, \ell_{\mathrm{t}}\right)$ & $\checkmark$ & - & - & - & - & - & - & - & - \\
\hline$\Delta R(\mathrm{t}, \mathrm{Z})$ & - & $\checkmark$ & $\checkmark$ & $\checkmark$ & - & $\checkmark$ & - & - & $\checkmark$ \\
\hline$\Delta \eta\left(\mathrm{Z}, j^{\prime}\right)$ & - & $\checkmark$ & - & - & - & - & - & $\checkmark$ & - \\
\hline$\Delta R$ between $\mathrm{t}$ and the closest lepton & - & $\checkmark$ & - & $\checkmark$ & - & - & - & - & - \\
\hline$\Delta R$ between $j^{\prime}$ and the closest lepton & - & - & - & - & - & - & - & $\checkmark$ & - \\
\hline$m_{3 \ell}$ & $\checkmark$ & - & - & - & $\checkmark$ & - & $\checkmark$ & - & $\checkmark$ \\
\hline$m_{\mathrm{T}}^{\mathrm{W}}$ & $\checkmark$ & $\checkmark$ & $\checkmark$ & - & - & - & - & - & $\checkmark$ \\
\hline$p_{\mathrm{T}}^{\mathrm{miss}}$ & $\checkmark$ & - & - & - & - & - & - & - & - \\
\hline Lepton asymmetry & $\checkmark$ & - & - & $\checkmark$ & $\checkmark$ & - & - & $\checkmark$ & - \\
\hline $\cos \theta_{Z}^{\star}$ & - & - & $\checkmark$ & - & - & $\checkmark$ & - & - & $\checkmark$ \\
\hline Max. $p_{\mathrm{T}}$ among jet pairs & - & - & - & - & - & - & $\checkmark$ & - & $\checkmark$ \\
\hline Max. DeepJet discriminant & $\checkmark$ & - & - & - & - & - & - & - & - \\
\hline b jet multiplicity & $\checkmark$ & - & - & - & - & - & - & - & - \\
\hline Three-momenta of the three leading leptons & $\checkmark$ & - & - & - & - & - & - & - & - \\
\hline Three-momenta of the three leading jets & $\checkmark$ & - & - & - & - & - & - & - & - \\
\hline DEEPJET discriminants of the three leading jets & $\checkmark$ & - & - & - & - & - & - & - & - \\
\hline Number of variables & 33 & 11 & 8 & 8 & 6 & 7 & 4 & 7 & 10 \\
\hline
\end{tabular}

Table 3. Input variables to the NN-SM and to the eight NN-EFTs. A dash indicates that the variable is not used. The three-momentum of an object includes the $p_{\mathrm{T}}, \eta$, and $\phi$ components of its momentum. The symbol $\ell_{\mathrm{t}}$ denotes the lepton produced in the decay of the top quark; $j^{\prime}$ denotes the recoiling jet; $b$ denotes the $\mathrm{b}$ jet associated with the leptonic top quark decay; $\left(\ell_{1}^{Z}, \ell_{2}^{Z}\right)$ denote the leptons produced in the $\mathrm{Z}$ boson decay; $\cos \theta_{\mathrm{Z}}^{\star}$ is the cosine of the angle between the direction of the $\mathrm{Z}$ boson in the detector reference frame, and the direction of the negatively-charged lepton from the $\mathbf{Z}$ boson decay in the rest frame of the $\mathbf{Z}$ boson. Other observables are defined in section 4 . 

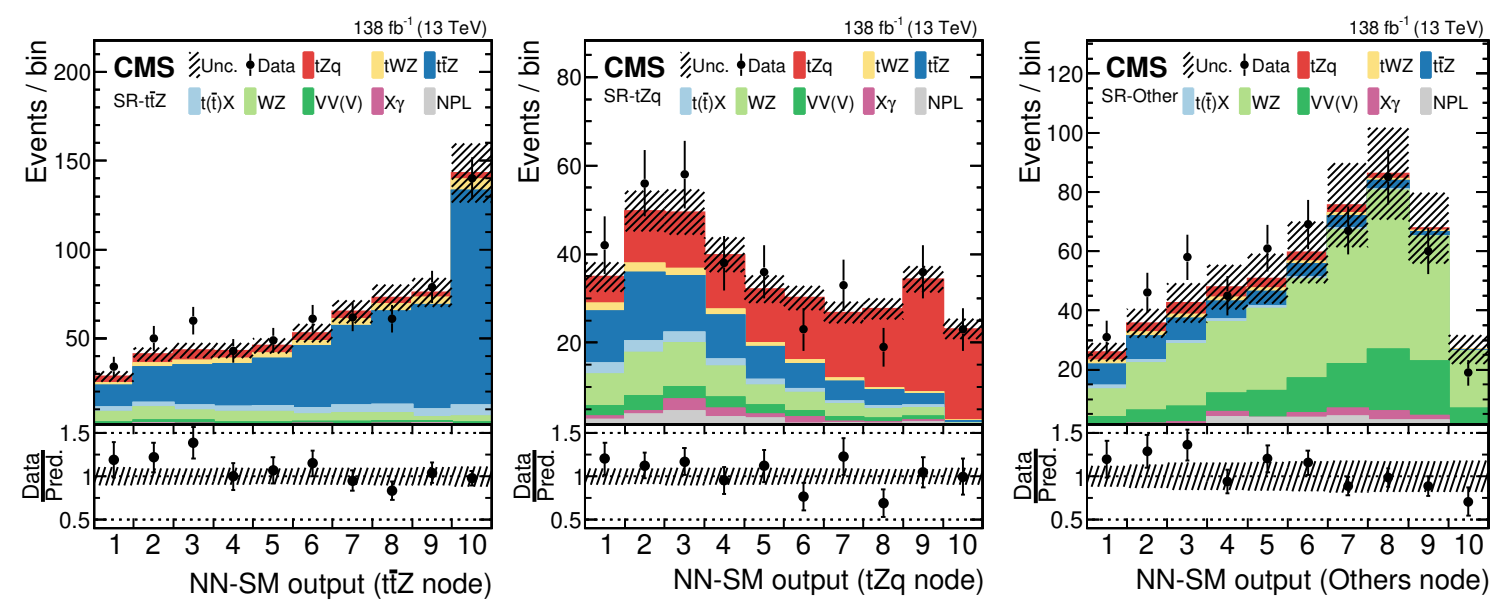

Figure 3. Pre-fit data-to-simulation comparisons for the distributions of the $t \bar{t} Z$ (left), tZq (middle), and Others (right) output nodes. For each distribution, only the events that have their maximum value in the corresponding output node are included. The lower panels display the ratios of the observed event yields to their predicted values. The hatched band represents the total uncertainty in the prediction.

SM events from events corresponding to the pure EFT contribution to the targeted signal process, as well as to distinguish among different classes of operators. However, the interference between EFT operators and the SM amplitude - and among EFT amplitudes were always either absent, or were voluntarily neglected in the design of these algorithms. This is because the shapes of the kinematic distributions due to pure EFT contributions are independent of the WCs, which makes it possible to train an algorithm on simulated samples whose kinematic properties are unambiguously defined. On the contrary, interference terms introduce a dependence of the shapes of the kinematic distributions on the WCs, which cannot be dealt with efficiently using the most commonly used, problem-specific algorithms. This is the first time that an LHC analysis uses machine-learning techniques that account for interference in the training phase to target EFT effects.

The NN-EFTs are binary NN classifiers trained to discriminate between the SM and EFT scenarios. We segment this challenging task by targeting individual WCs with separate trainings, and by targeting the $\mathrm{tZq}$ and $\mathrm{t} \overline{\mathrm{t} Z}$ signals separately, since both processes lead to significantly different event topologies. As anticipated, we find that for WC values close to the current exclusion limits, the $\mathcal{O}_{\varphi \mathrm{Q}}^{-}$and $\mathcal{O}_{\varphi \mathrm{t}}$ operators have negligible impacts on the shapes of the kinematic distributions of the tZq and t€Z processes [20]. We therefore do not train dedicated classifiers for these cases, but we rely on the sensitivity of this analysis to the signal cross sections to constrain these WCs. Additional classifiers, referred to explicitly as "NN-5D", are trained with events sampled over the phase space spanned by $c_{\mathrm{tZ}}, c_{\mathrm{tW}}$, and $c_{\varphi \mathrm{Q}}^{3}$, and will be used to constrain multiple WCs simultaneously. Consequently, eight NN-EFTs are trained and labeled according to the $\mathrm{WC}(\mathrm{s})$ and signal process

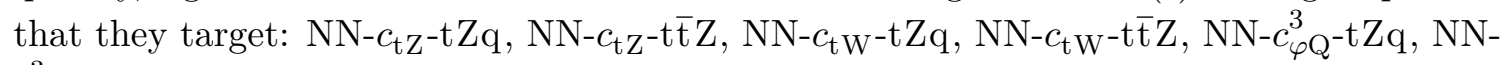

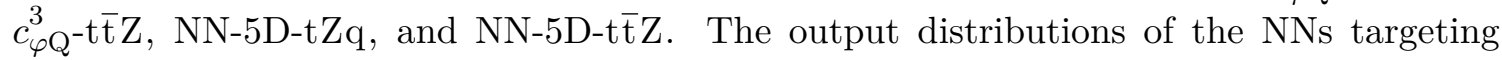


either the $\mathrm{tZq}$ or $\mathrm{t} \overline{\mathrm{ZZ}}$ process are ultimately used to extract the signal in the SR-tZq or

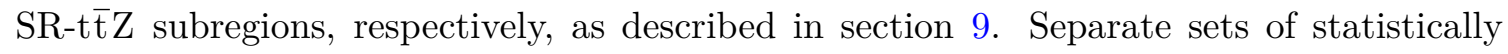
independent samples are used to perform the trainings and the signal extraction.

Each NN-EFT classifier is trained on a range of distinct EFT scenarios. The corresponding subsets of training events are sampled uniformly over the range $[-5,5]$ for $c_{\mathrm{tZ}}$ and $c_{\mathrm{tW}}$, and $[-10,10]$ for $c_{\varphi \mathrm{Q}}^{3}$. These ranges for the training hypotheses are chosen to be broader than the existing direct constraints on the corresponding WCs. This avoids biasing the trainings on preexisting results and may help the classifiers learn the characteristic features associated with new interactions, since these features are more prominent at larger WC values. The exact definition for these ranges has only a minor impact on the classification performance. We rely upon the capability of these NNs to learn from training events corresponding to various hypotheses, and to interpolate between these events to construct an abstract model optimized to separate reconstructed events that are either SM- or EFT-like.

The NN-EFTs include three hidden layers, each with 50 rectified linear units. The sigmoid activation function is used for the single output node. The sets of input variables are defined independently for each classifier and are listed in table 3. The choice of input variables is informed by generator-level studies of their expected sensitivities, as well as by their correlations, importance rankings, and modeling. Multiple tests were performed using different architectures, activation functions, optimizers, and learning and dropout rates, and the configuration performing best on a set of simulated events not used during the training phase was retained.

\section{Systematic uncertainties}

Many different sources of systematic uncertainty may alter the event yields, the shapes of the observables used in the final fits to data, or both. They are treated as correlated between the different regions and data-taking periods, unless stated otherwise. A summary of the sources of systematic uncertainty included in the measurements is provided in table 4 .

\subsection{Experimental uncertainties}

- Integrated luminosity. The integrated luminosities of the 2016, 2017, and 2018 datataking periods are individually known with uncertainties in the $1.2-2.5 \%$ range [2628]. The total Run 2 integrated luminosity has an uncertainty of $1.6 \%$.

- Trigger efficiency. The combination of single-, double-, and triple-lepton trigger algorithms used in this analysis achieves a trigger efficiency of nearly $100 \%$ for events passing the SR or CR selection criteria, both in data and simulation, and therefore no correction is applied. However, a $2 \%$ uncertainty is assigned to the event yields of the MC samples independently for each year to account for the statistical uncertainty in the efficiency measurement in data.

- Pileup. Event weights are corrected to account for differences in the pileup distributions between data and MC samples, before any event selection is applied. To 
estimate the corresponding uncertainty, the pp inelastic cross section is varied by $\pm 4.6 \%[76]$ and the variations are propagated to the event weights.

- Lepton identification and isolation efficiencies. Event weights are corrected to account for differences in the lepton identification and isolation efficiencies between data and simulation, as a function of the lepton $p_{\mathrm{T}}$ and $\eta$. These corrections are varied independently for electrons and muons, within the uncertainties in the corresponding measurements.

- $b$ jet identification efficiency. Event weights are corrected to make the simulated distribution of the DEEPJET discriminant match that measured in the data. These corrections are parameterized with the jet $p_{\mathrm{T}}$ and $\eta$, and the corresponding uncertainties are measured in $t \bar{t}+$ jets and multijet events [63]. Several sources of uncertainty are considered, which are related to the contaminations and statistical fluctuations in the measurement regions of the corrections. Uncertainties that are statistical in nature are uncorrelated between the years.

- Jet energy and missing transverse momentum. The jet energy scale is measured with an uncertainty of a few percent, depending on the jet $p_{\mathrm{T}}$ and $\eta$, in $\mathrm{Z} / \gamma^{\star} \rightarrow$ ee, $\mathrm{Z} / \gamma^{\star} \rightarrow \mu \mu, \gamma+$ jets, dijet, and multijet events [62]. The resulting effect on signal and background distributions is evaluated by varying the energies of jets in simulated events within their uncertainties, recalculating all kinematic observables, and reapplying the event selection criteria. This effect is split between several subsources of uncertainty that are either correlated or uncorrelated between the years. The uncertainties in the jet energy resolution and in the vector $\vec{p}_{\mathrm{T}}^{\text {miss }}$ are evaluated in a similar way, and have smaller effects than the uncertainty in the jet energy scale. These uncertainties are treated as uncorrelated between the years.

- L1 ECAL trigger inefficiency. A gradual shift in the timing of the inputs of the ECAL L1 trigger in the forward region $(|\eta|>2.4)$ constitutes an additional source of inefficiency in the 2016 and 2017 data-taking periods [30]. Dedicated event weights are applied to simulated events to account for this effect and are varied within their uncertainties.

\subsection{Theoretical uncertainties}

Several sources of theoretical uncertainty related to the modeling of the signal samples are included. Except for the uncertainties in the SM cross sections, these uncertainties impact both the event yields through changes in signal acceptance, and the shapes of the observables used for the signal extraction.

- $P D F$ and $Q C D$ coupling. The uncertainty in the PDF prediction is estimated by reweighting signal events according to 100 eigenvectors of the NNPDF3.1 PDF set. The total uncertainty is measured following the PDF4LHC recommendations [77]. The value of the $\mathrm{QCD}$ coupling $\alpha_{\mathrm{S}}$ is also varied independently within its uncertainty. 
- Renormalization and factorization scales. The effect of missing higher-order corrections on the distributions of the discriminating observables is estimated by varying the renormalization and factorization scales $\left(\mu_{\mathrm{R}}\right.$ and $\left.\mu_{\mathrm{F}}\right)$ up and down by a factor of two with respect to their nominal values, avoiding cases in which the two variations are done in opposite directions.

- SM cross sections. We assign uncertainties to the SM cross sections of the signal processes based on theoretical predictions computed at NLO accuracy. We sum in quadrature the uncertainties in the PDF, the renormalization scale, and the factorization scale. This results in a normalization-only uncertainty of ${ }_{-11.6}^{+10.0} \%$ for the $t \bar{t} Z$ process [53], and of $\pm 3.3 \%$ for the $\mathrm{tZq}$ process [54]. As there is yet no experimental evidence for the existence of the tWZ process, we assign a more conservative uncertainty of $20 \%$ to its cross section.

- Parton shower. The uncertainty in the PS simulation is estimated by varying the renormalization scale up and down by a factor of two with respect to its nominal value, independently for initial- and final-state radiation (ISR and FSR). The effect of the ISR uncertainty is uncorrelated between the signal processes.

- Additional radiation. Comparisons between the SM tZq sample (generated at NLO accuracy) and the tZq sample used for the signal extraction (generated at LO without an extra final-state parton) show good agreement, except for a discrepancy in the jet multiplicity distribution that is not covered by other uncertainties. We include this effect by adding a systematic uncertainty based on these differences in bins of jet multiplicity, which only affects the shapes of observables for the $\mathrm{tZq}$ signal.

\subsection{Background uncertainties}

- Irreducible backgrounds. Uncertainties are assigned to the cross section predictions of all irreducible background processes. We assign uncertainties of $10 \%$ to the cross sections of the WZ [78], $\mathrm{VV}(\mathrm{V})[79,80]$, and $\mathrm{X} \gamma$ [81, 82] processes, and of $20 \%$ to those of $\mathrm{t}(\overline{\mathrm{t}}) \mathrm{X}$ processes $[60,83]$. An additional extrapolation uncertainty of $6 \%$ is applied to WZ events that enter any of the signal regions, to account for differences in the multiplicity of heavy-flavor jets between the WZ CR and SRs, as estimated from simulations.

- Misidentified-lepton probability estimation. The misidentification probability measurements described in section 6 are affected by several sources of uncertainty related to the limited amount of data in the measurement region, the subtraction of the prompt lepton contamination, and potential differences in kinematic properties of NPL candidates between the measurement and application regions. The weights obtained with this procedure are varied independently for electrons and muons within each uncertainty. Since these uncertainties were estimated in the context of ref. [60], we apply an additional 30\% uncertainty to the event yield of the NPL background to account for differences in the definitions of the application regions of this analysis. 


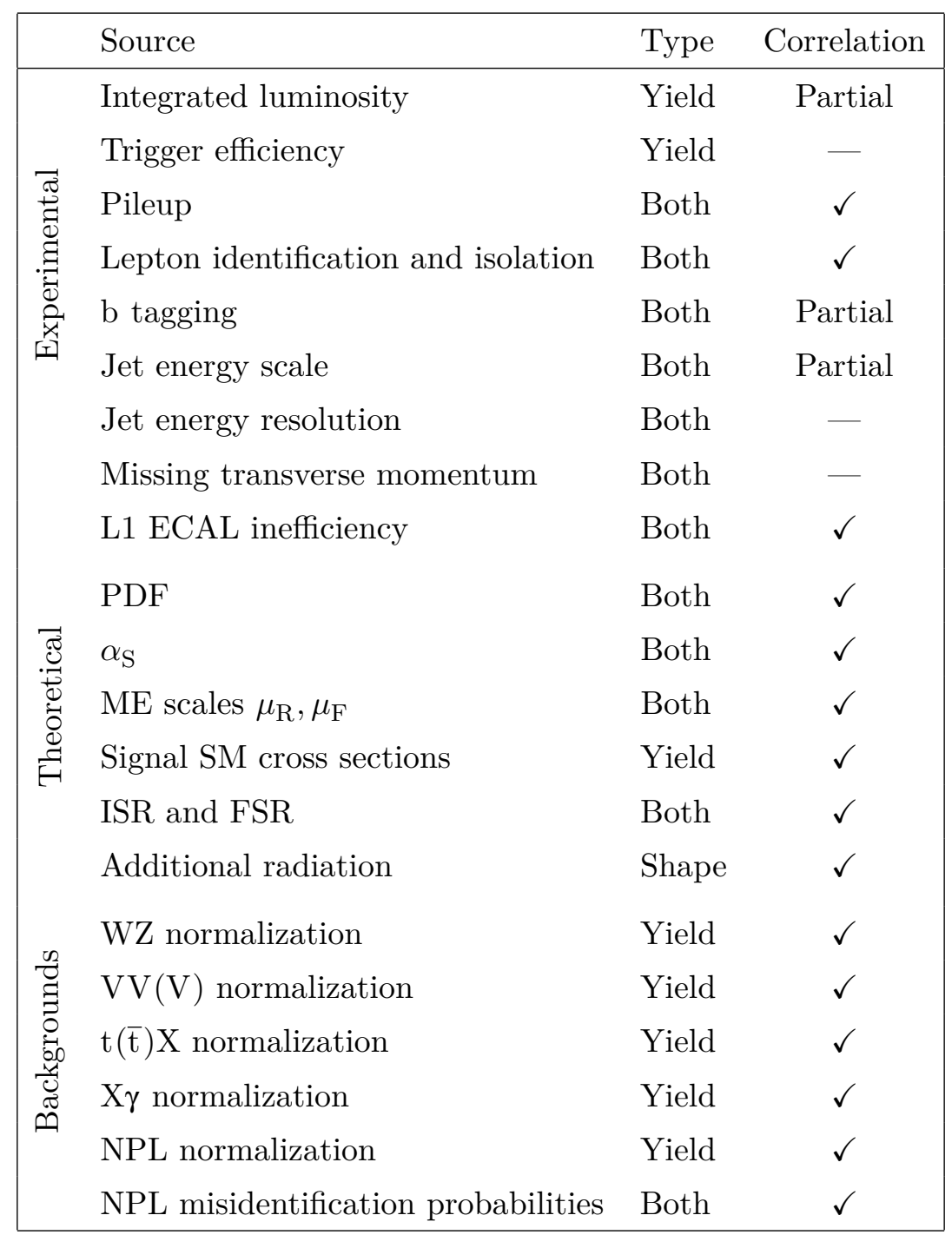

Table 4. Summary of the different sources of systematic uncertainty included in the measurements. The first column indicates the source of the uncertainty. The second column indicates whether the source affects the event yields, the shapes of the observables, or both. In the third column, the symbols " $\checkmark$ " and "_-" indicate $100 \%$ and $0 \%$ correlations between the data-taking periods, respectively.

\section{Signal extraction}

The statistical analysis of the data relies upon the construction of a binned likelihood function $\mathcal{L}($ data $\mid \theta, \nu)$ that incorporates all the necessary information regarding the different model parameters. The WCs constitute the parameters of interest, denoted by $\theta$, and all the systematic uncertainties defined in section 8 are treated as nuisance parameters, denoted by $\nu$. The parameterization of the signal event weights with the WCs makes it possible to vary the event yields in all bins, to describe any point in the parameter space spanned by the WCs. Statistical fluctuations due to the finite number of simulated events are incorporated into the likelihood function via the approach described in refs. [84, 85]. 
The compatibility of given values of the model parameters with the data is quantified by performing a maximum likelihood fit in which the negative log-likelihood (NLL) function $-2 \ln (\mathcal{L})$ is minimized and the nuisance parameters are profiled, simultaneously in the six regions (four SRs and two CRs) for the three years.

We perform a one-dimensional (1D) likelihood scan for each WC by repeating the maximum likelihood fit in steps of that WC, while the four other WCs are fixed to their SM values of zero. Any point in the scan is described by the difference $-2 \Delta \ln (\mathcal{L})$ between its NLL value and that of the global minimum. The boundaries of the 68 and $95 \%$ confidence level (CL) confidence intervals are defined as the intersections of the NLL function with values of 1 and 3.84, respectively. Five individual 1D likelihood scans are thus required to construct confidence intervals for all five WCs. In addition, we perform two-dimensional (2D) likelihood scans in which two WCs are scanned simultaneously, while the three remaining WCs are fixed to zero; the boundaries of the 68 and 95\% CL confidence intervals are defined as the intersections of the NLL function with values of 2.30 and 5.99, respectively. The corresponding plots shown in section 10 illustrate the correlations between pairs of WCs. Finally, we also perform a single 5D maximum likelihood fit in which all five WCs are treated as parameters of interest, and we compute the corresponding 95\% CL confidence intervals.

Different observables are used to extract the signal in the SR-tZq and SR-t $\bar{Z} Z$, depending on the number of parameters of interest (one, two, or five) and on the WCs that are probed, while the observables used in all other regions are the same for all fits. In the SR-Others we use the $m_{\mathrm{T}}^{\mathrm{W}}$ distribution since it provides good separation between the main background processes in this region. We perform simple counting experiments in the SR-t $\bar{t} Z-4 \ell$, WZ CR, and ZZ CR regions, as they are already very pure in their target processes and do not require any further discrimination. The distributions of the different NN classifiers are used to extract the signal in the SR-tZq and SR-tẼ $Z$ subregions, which drive almost entirely the sensitivity of this analysis. For the $1 \mathrm{D}$ fits to the $c_{\mathrm{tZ}}, c_{\mathrm{tW}}$, and $c_{\varphi \mathrm{Q}}^{3}$ coefficients, we use the distributions of the dedicated NN-EFT classifiers. For the 1D fits to the $c_{\varphi \mathrm{Q}}^{-}$and $c_{\varphi \mathrm{t}}$ coefficients, we use the distributions of the $\mathrm{tZq}$ and $\mathrm{t} \overline{\mathrm{t}} \mathrm{Z}$ output nodes of the NN-SM classifier. The good discrimination power of the latter observables between the relevant SM processes improves the sensitivity to the signal event yields. The $2 \mathrm{D}$ and $5 \mathrm{D}$ fits are performed to the distributions of the NN-5D classifiers, which are trained to learn the features associated with several EFT operators simultaneously. The observables used in the different fits are listed for each region in table 5 .

\section{Results}

The distributions that are common to all fits are shown in figure 4, after performing the fit (post-fit) in which all five WCs are treated as free parameters. The post-fit distributions of the observables used in the SR-tZq and SR-t产Z for the 5D fit and for the 1D fits to the

$c_{\mathrm{tZ}}, c_{\mathrm{tW}}$, and $c_{\varphi \mathrm{Q}}^{3}$ coefficients, are shown in figures 5-6. The post-fit distributions of the

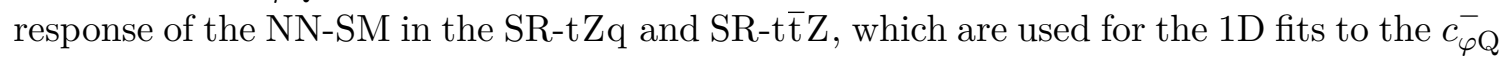
and $c_{\varphi \mathrm{t}}$ coefficients, are not shown because they do not exhibit any shape discrimination for the considered EFT scenarios (as explained in section 7.2), and because they are very 


\begin{tabular}{|c|c|c|c|c|}
\hline \multirow[t]{2}{*}{ Fit configuration } & \multicolumn{4}{|c|}{ Region } \\
\hline & SR-tZq & SR-t̄̃Z & SR-Others & 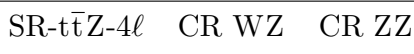 \\
\hline $1 \mathrm{D} c_{\mathrm{tZ}}$ & $\mathrm{NN}-c_{\mathrm{tZ}}-\mathrm{tZq}$ & $\mathrm{NN}-c_{\mathrm{tZ}}-\mathrm{t} \overline{\mathrm{t}} \mathrm{Z}$ & \multirow{6}{*}{$m_{\mathrm{T}}^{\mathrm{W}}$} & \multirow{6}{*}{ Counting experiments } \\
\hline $1 \mathrm{D} c_{\mathrm{tW}}$ & $\mathrm{NN}-c_{\mathrm{tW}}{ }^{-\mathrm{tZ}} \mathrm{q}$ & $\mathrm{NN}-c_{\mathrm{tW}}-\mathrm{t} \overline{\mathrm{t} Z}$ & & \\
\hline $1 \mathrm{D} c_{\varphi \mathrm{Q}}^{3}$ & $\mathrm{NN}-c_{\varphi \mathrm{Q}^{-\mathrm{tZ}}}^{3}$ & $\mathrm{NN}-c_{\varphi \mathrm{Q}^{3}}^{3} \mathrm{t \overline { \textrm {t } } \mathrm { Z }}$ & & \\
\hline $1 \mathrm{D} c_{\varphi \mathrm{Q}}^{-}$ & NN-SM (tZq node) & NN-SM (tēZ node) & & \\
\hline $1 \mathrm{D} c_{\varphi \mathrm{t}}$ & NN-SM (tZq node) & NN-SM (t̄̄Z node) & & \\
\hline $2 \mathrm{D}$ and $5 \mathrm{D}$ & NN-5D-tZq & $\mathrm{NN}-5 \mathrm{D}-\mathrm{t} \overline{\mathrm{t} Z}$ & & \\
\hline
\end{tabular}

Table 5. Observables used in each region for the different fits. The NN-SM is trained to separate different SM processes, while the other NNs are trained to identify new effects arising from one or more EFT operators, as described in section 7.
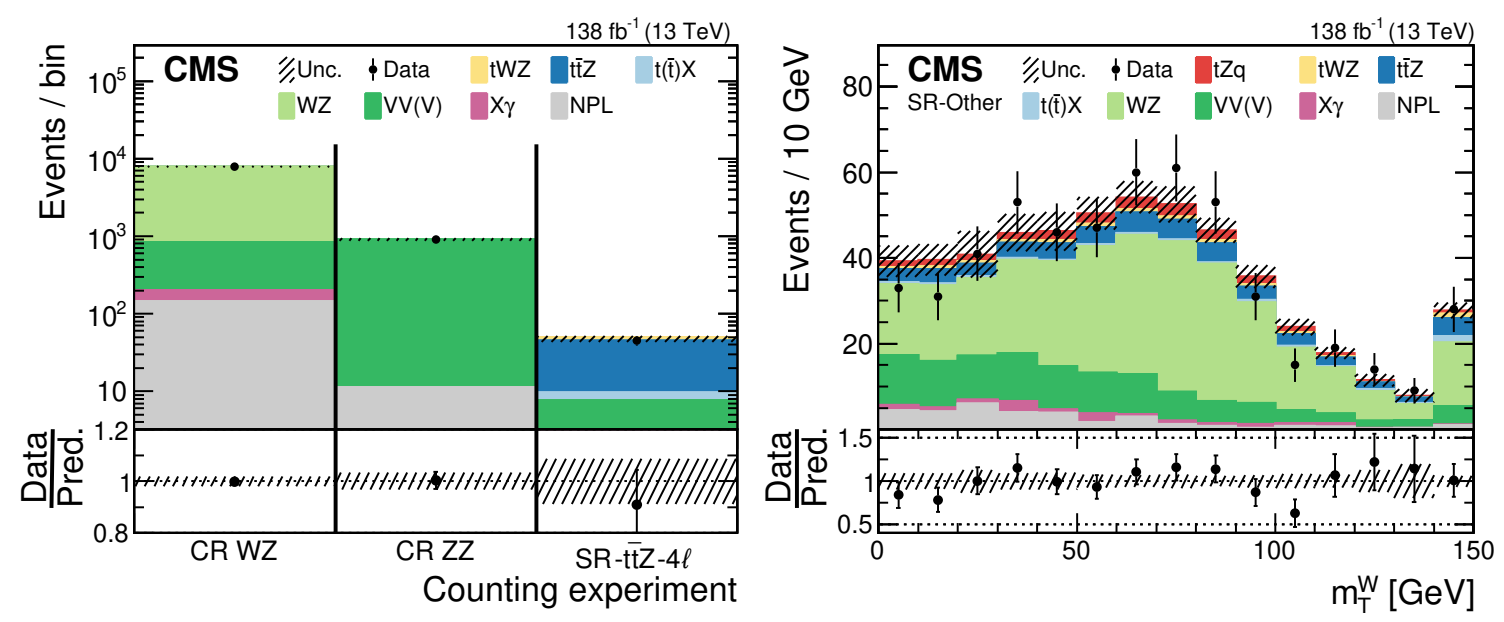

Figure 4. Post-fit data-to-simulation comparisons for the distributions that are common to all fits,

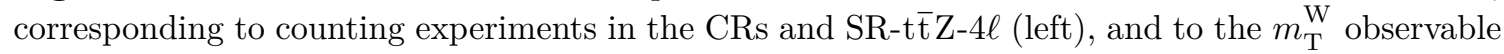
in the SR-Others (right), after the 5D fit. The lower panels display the ratios of the observed event yields to their post-fit expected values. Overflows are included in the last bin of the right figure.

similar to the corresponding pre-fit distributions shown in figure 3. We observe good agreement between data and simulation in all distributions, and illustrate the separation powers of the different NN-EFT classifiers for benchmark EFT scenarios.

The expected and observed 95\% CL confidence intervals obtained from the 1D and 5D fits are provided in table 6. All intervals include the SM expectations of zero for the WC values. The optimal combination of the available kinematic information by the NNEFT classifiers significantly reduces the widths of the confidence intervals, as estimated by repeating the fits when performing simple counting experiments in all regions. The widths of the expected $1 \mathrm{D}$ confidence intervals for $c_{\mathrm{tW}}, c_{\mathrm{tZ}}$, and $c_{\varphi \mathrm{Q}}^{3}$ are reduced by about 55 , 40 , and $20 \%$, respectively. Those for $c_{\varphi \mathrm{Q}}^{-}$and $c_{\varphi \mathrm{t}}$ change only marginally, as anticipated since these WCs mainly affect the signal cross sections. The widths of the expected 5D confidence intervals for $c_{\mathrm{tW}}, c_{\varphi \mathrm{Q}}^{3}, c_{\mathrm{tZ}}, c_{\varphi \mathrm{Q}}^{-}$, and $c_{\varphi \mathrm{t}}$ are reduced by about $70,70,60,35$, and $20 \%$, respectively. The corresponding observed intervals exhibit similar or slightly 

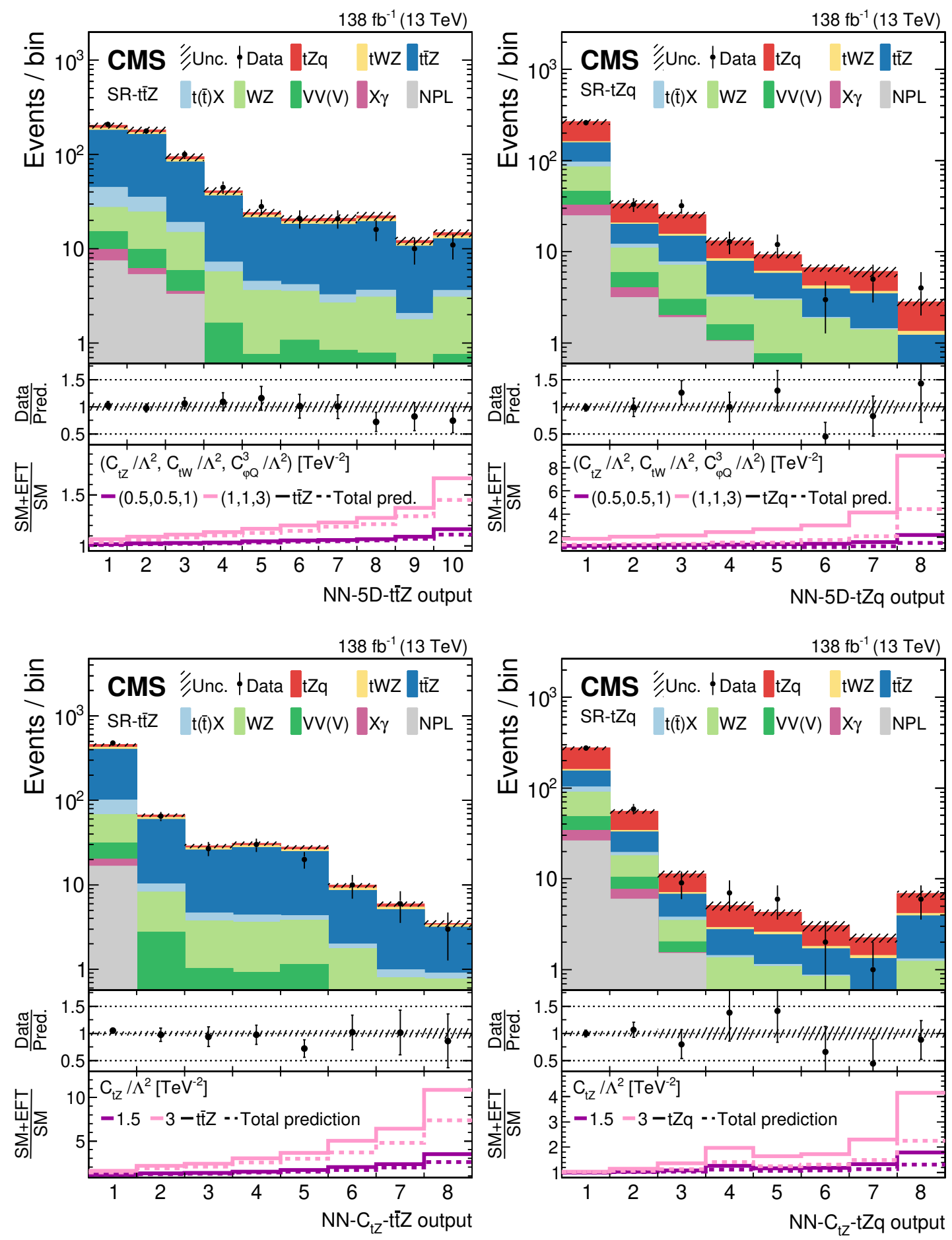

Figure 5. Post-fit data-to-simulation comparisons for the distributions used in the SR-t言 (left) and SR-tZq (right), for the $5 \mathrm{D}$ fit (upper) and for the $1 \mathrm{D}$ fit to $c_{\mathrm{tZ}}$ (lower). The middle panels display the ratios of the observed event yields to their post-fit expected values. For each region, the lower panel shows the change of the event yield in each bin with respect to the SM post-fit expectation for two benchmark EFT scenarios, both for the main signal process in the region (thick lines) and for the total prediction (dashed lines). 

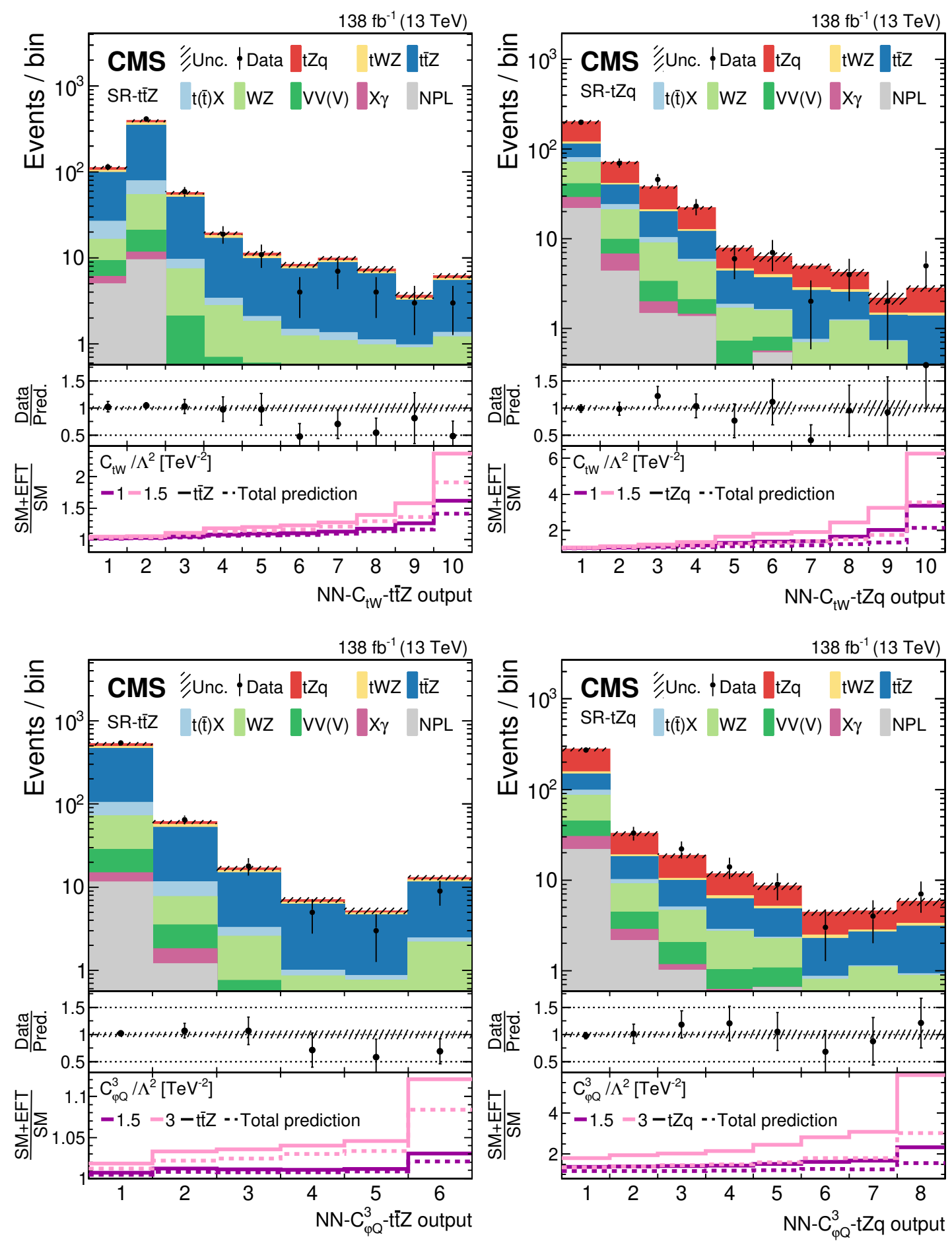

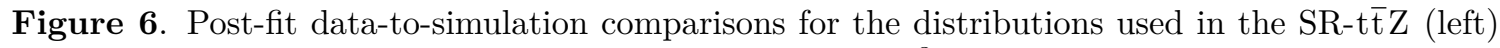
and SR-tZq (right), for the $1 \mathrm{D}$ fits to $c_{\mathrm{tW}}$ (upper) and to $c_{\varphi \mathrm{Q}}^{3}$ (lower). The middle panels display the ratios of the observed event yields to their post-fit expected values. For each region, the lower panel shows the change of the event yield in each bin with respect to the SM prediction for two benchmark EFT scenarios, both for the main signal process in the region (thick lines) and for the total prediction (dashed lines). 


\begin{tabular}{|c|c|c|c|c|}
\hline \multirow{3}{*}{$\begin{array}{l}\mathrm{WC} / \Lambda^{2} \\
{\left[\mathrm{TeV}^{-2}\right]}\end{array}$} & \multicolumn{4}{|c|}{ 95\% CL confidence intervals } \\
\hline & \multicolumn{2}{|c|}{ Other WCs fixed to SM } & \multicolumn{2}{|l|}{$5 \mathrm{D}$ fit } \\
\hline & Expected & Observed & Expected & Observed \\
\hline$c_{\mathrm{tZ}}$ & {$[-0.97,0.96]$} & {$[-0.76,0.71]$} & {$[-1.24,1.17]$} & {$[-0.85,0.76]$} \\
\hline$c_{\mathrm{tW}}$ & {$[-0.76,0.74]$} & {$[-0.52,0.52]$} & {$[-0.96,0.93]$} & {$[-0.69,0.70]$} \\
\hline$c_{\varphi \mathrm{Q}}^{3}$ & {$[-1.39,1.25]$} & {$[-1.10,1.41]$} & {$[-1.91,1.36]$} & {$[-1.26,1.43]$} \\
\hline$c_{\varphi \mathrm{Q}}^{-}$ & {$[-2.86,2.33]$} & {$[-3.00,2.29]$} & {$[-6.06,14.09]$} & {$[-7.09,14.76]$} \\
\hline$c_{\varphi \mathrm{t}}$ & {$[-3.70,3.71]$} & {$[-21.65,-14.61] \bigcup[-2.06,2.69]$} & {$[-16.18,10.46]$} & {$[-19.15,10.34]$} \\
\hline
\end{tabular}

Table 6. Expected and observed 95\% CL confidence intervals for all WCs. The intervals in the first and second columns are obtained by scanning over a single WC, while fixing the other WCs to their SM values of zero. The intervals in the third and fourth columns are obtained by performing a 5D fit in which all five WCs are treated as free parameters. As explained in section 9, the 1D intervals are obtained from separate fits to different observables in the SR-tZq and SR-tt Z, while the $5 \mathrm{D}$ intervals are obtained from a single fit.

larger improvements. This indicates that leveraging kinematic information becomes all the more important when aiming to constrain multiple WCs simultaneously, as it helps with disentangling effects from different EFT operators that have an interplay.

The impacts from different groups of sources of systematic uncertainty on each individual $\mathrm{WC}$ are listed in table 7 . We find that the uncertainties in the $c_{\mathrm{tZ}}$ and $c_{\mathrm{tW}}$ coefficients are primarily due to the limited number of data events in the most sensitive bins of the SR-3 $\ell$ distributions. Conversely, the measurements of the $c_{\varphi \mathrm{Q}}^{3}, c_{\varphi \mathrm{Q}}^{-}$, and $c_{\varphi \mathrm{t}}$ coefficients are limited by systematic uncertainties, with those in the signal and background cross sec-

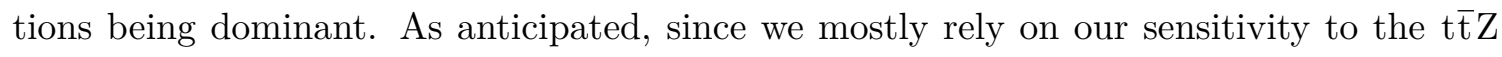
event yield to constrain the $c_{\varphi \mathrm{Q}}^{-}$and $c_{\varphi \mathrm{t}}$ coefficients, the corresponding confidence intervals

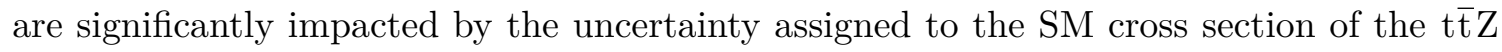
process.

One-dimensional likelihood scans are shown for all WCs in figure 7. The 1D likelihood scan of $c_{\varphi \mathrm{t}}$ exhibits a double-minima structure; while the data favor the negative minimum, they are compatible with the SM at 95\% CL. It was verified with MC pseudo-experiments generated under the SM hypothesis that the negative minimum is favored about $20 \%$ of the time due to statistical fluctuations. Two-dimensional likelihood scans are shown in figure 8 for the pairs of WCs that are most correlated. 


\begin{tabular}{|lrrrrr|}
\hline Source & $c_{\mathrm{tZ}}$ & $c_{\mathrm{tW}}$ & $c_{\varphi \mathrm{Q}}^{3}$ & $c_{\varphi \mathrm{Q}}^{-}$ & $c_{\varphi \mathrm{t}}$ \\
\hline tZq normalization & $<0.1$ & $<0.1$ & 1.2 & 0.1 & 0.8 \\
tẼZ normalization & 0.6 & $<0.1$ & 0.4 & 37 & 38 \\
tWZ normalization & 0.1 & 0.1 & $<0.1$ & 0.7 & 2.1 \\
Background normalizations & $<0.1$ & $<0.1$ & 6.9 & 3.6 & 6.8 \\
NPL background estimation & 1.4 & 0.2 & 5.6 & 0.3 & 3.8 \\
Jet energy scale & $<0.1$ & $<0.1$ & 0.8 & 0.7 & 2.3 \\
Jet energy resolution & $<0.1$ & $<0.1$ & $<0.1$ & $<0.1$ & 1.4 \\
$p_{\mathrm{T}}^{\text {miss }}$ & $<0.1$ & $<0.1$ & $<0.1$ & $<0.1$ & 0.2 \\
b tagging & $<0.1$ & $<0.1$ & 0.9 & 2.0 & 0.3 \\
Other (experimental) & $<0.1$ & $<0.1$ & 1.6 & 0.8 & 0.6 \\
Lepton identification and isolation & 0.4 & 0.4 & 1.2 & 2.2 & 0.8 \\
Theory & 2.1 & 1.1 & 0.4 & 0.9 & 0.9 \\
\hline
\end{tabular}

Table 7. Impacts from different groups of sources of systematic uncertainty on each individual WC. To estimate the impact of a given group, the corresponding sources of systematic uncertainty are excluded, the 1D fits to the data are repeated, and the reduction in the width of the confidence interval is quoted for each WC. The values are given in percent.

\section{Summary}

A search for new top quark interactions has been performed within the framework of an effective field theory (EFT) using the associated production of either one or two top quarks with a $\mathrm{Z}$ boson in multilepton final states. The data sample corresponds to an integrated luminosity of $138 \mathrm{fb}^{-1}$ of proton-proton collisions at $\sqrt{s}=13 \mathrm{TeV}$ collected by the CMS experiment. Five dimension-six operators modifying the electroweak interactions of the top quark were considered. The event yields and kinematic properties of the signal processes were parameterized with Wilson coefficients (WCs) describing the interaction strengths of these operators.

A multivariate analysis relying upon machine-learning techniques was designed to enhance the sensitivity to effects arising from the EFT operators. A multiclass neural network was trained to distinguish between standard model (SM) processes and was used to define three subregions enriched in $\mathrm{tZq}, \mathrm{t} \overline{\mathrm{ZZ}}$, and background events. Additional neural networks were trained to separate events generated according to the SM from events generated with nonzero WC values, and were used to construct optimal observables. This is the first time that machine-learning techniques accounting for the interference between EFT operators and the SM amplitude have been used in an LHC analysis.

Results were extracted from a simultaneous fit to data in six event categories. Two confidence intervals were determined for each WC, one keeping the other WCs fixed to zero and the other treating all five WCs as free parameters. Two-dimensional contours were produced for pairs of WCs to illustrate their correlations. All results are consistent with the SM at $95 \%$ confidence level. 

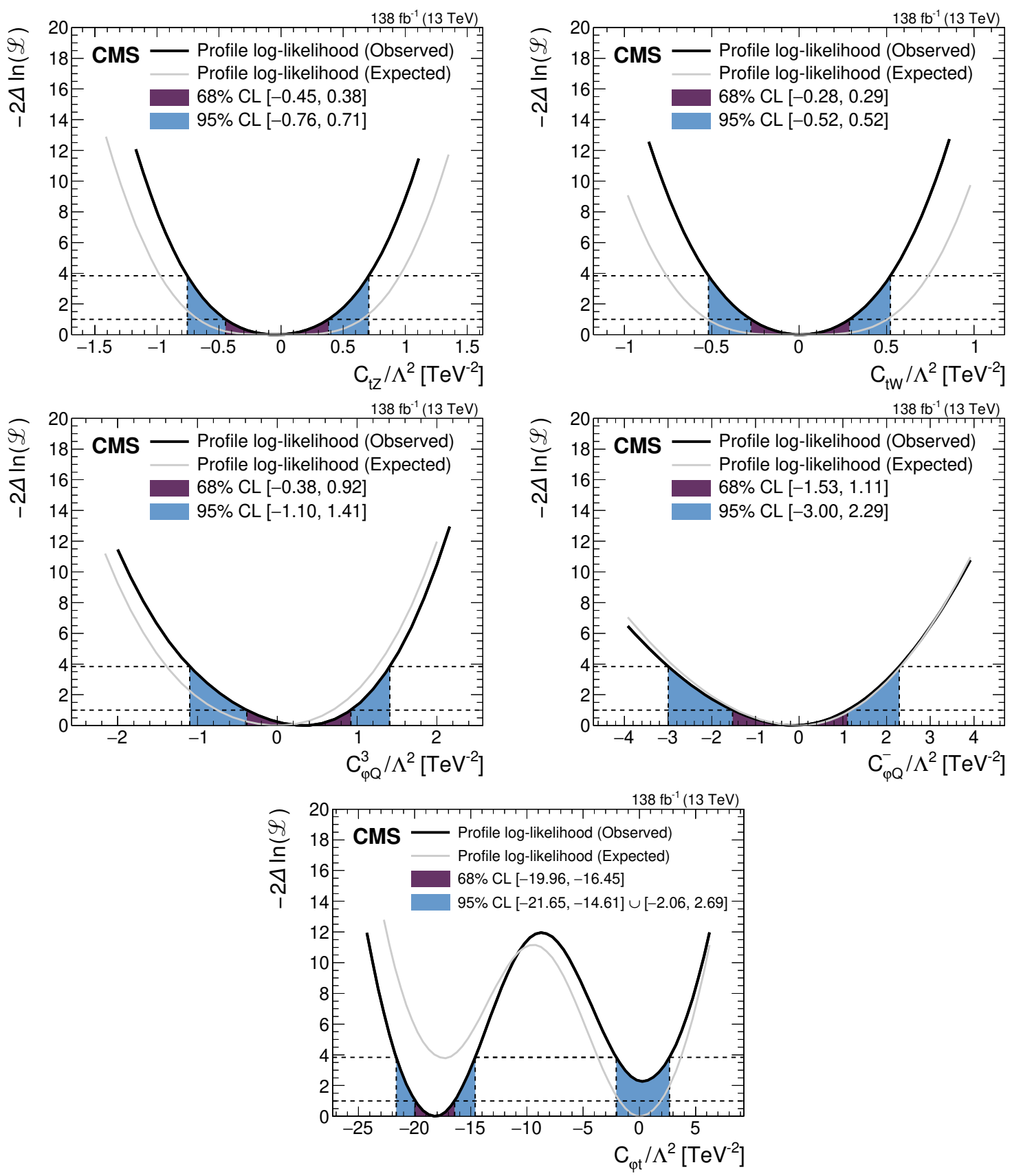

Figure 7. Observed (thick black lines) and expected (thin gray lines) one-dimensional scans of the negative log-likelihood as a function of each of the five WCs, while fixing the other WCs to their SM values of zero. The 68 and $95 \%$ CL confidence intervals are indicated by the colored areas. 

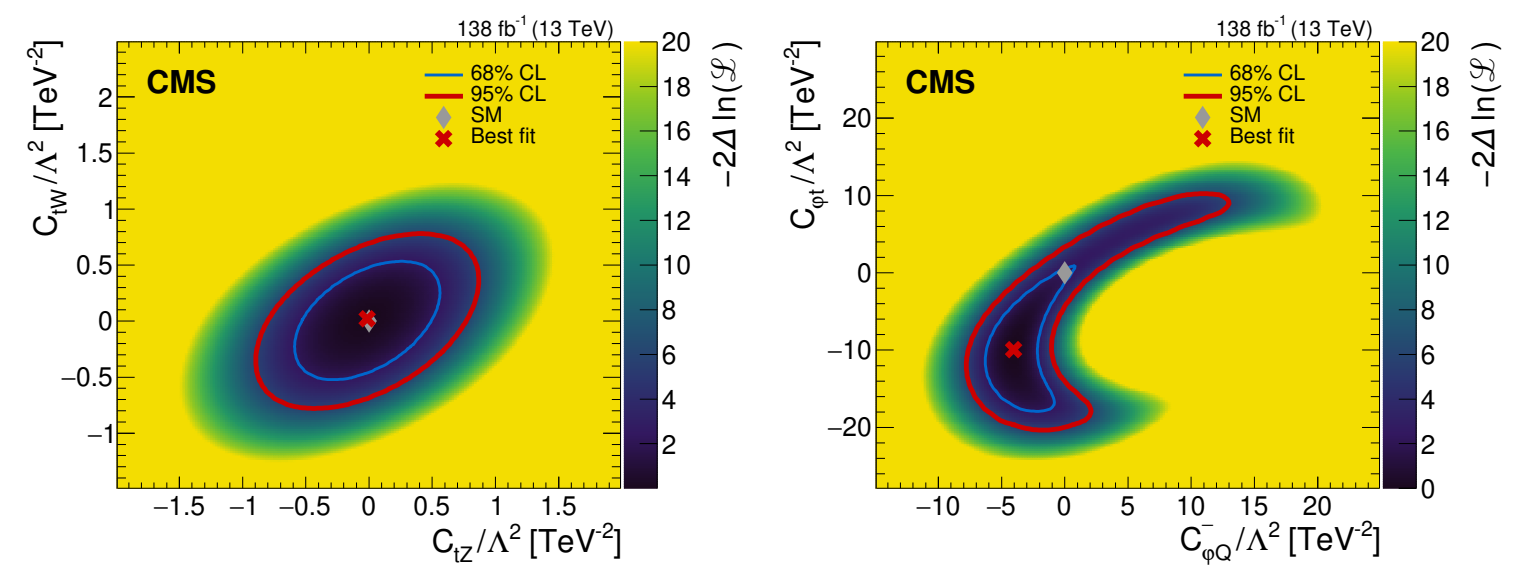

Figure 8. Two-dimensional scans of the negative log-likelihood as a function of $c_{\mathrm{tZ}}$ and $c_{\mathrm{tW}}$ (left), or as a function of $c_{\varphi \mathrm{Q}}^{-}$and $c_{\varphi \mathrm{t}}$ (right), while fixing the other WCs to their SM values of zero. The SM and best fit points are indicated by diamond- and cross-shaped markers, respectively. The thin blue line and thick red line represent the 68 and 95\% CL contours, respectively.

\section{Acknowledgments}

We congratulate our colleagues in the CERN accelerator departments for the excellent performance of the LHC and thank the technical and administrative staffs at CERN and at other CMS institutes for their contributions to the success of the CMS effort. In addition, we gratefully acknowledge the computing centers and personnel of the Worldwide LHC Computing Grid and other centers for delivering so effectively the computing infrastructure essential to our analyses. Finally, we acknowledge the enduring support for the construction and operation of the LHC, the CMS detector, and the supporting computing infrastructure provided by the following funding agencies: BMBWF and FWF (Austria); FNRS and FWO (Belgium); CNPq, CAPES, FAPERJ, FAPERGS, and FAPESP (Brazil); MES (Bulgaria); CERN; CAS, MoST, and NSFC (China); MINCIENCIAS (Colombia); MSES and CSF (Croatia); RIF (Cyprus); SENESCYT (Ecuador); MoER, ERC PUT and ERDF (Estonia); Academy of Finland, MEC, and HIP (Finland); CEA and CNRS/IN2P3 (France); BMBF, DFG, and HGF (Germany); GSRT (Greece); NKFIA (Hungary); DAE and DST (India); IPM (Iran); SFI (Ireland); INFN (Italy); MSIP and NRF (Republic of Korea); MES (Latvia); LAS (Lithuania); MOE and UM (Malaysia); BUAP, CINVESTAV, CONACYT, LNS, SEP, and UASLP-FAI (Mexico); MOS (Montenegro); MBIE (New Zealand); PAEC (Pakistan); MSHE and NSC (Poland); FCT (Portugal); JINR (Dubna); MON, RosAtom, RAS, RFBR, and NRC KI (Russia); MESTD (Serbia); SEIDI, CPAN, PCTI, and FEDER (Spain); MOSTR (Sri Lanka); Swiss Funding Agencies (Switzerland); MST (Taipei); ThEPCenter, IPST, STAR, and NSTDA (Thailand); TUBITAK and TAEK (Turkey); NASU (Ukraine); STFC (United Kingdom); DOE and NSF (U.S.A.).

Individuals have received support from the Marie-Curie program and the European Research Council and Horizon 2020 Grant, contract Nos. 675440, 724704, 752730, 758316, 765710, 824093, and COST Action CA16108 (European Union); the Leventis Foundation; 
the Alfred P. Sloan Foundation; the Alexander von Humboldt Foundation; the Belgian Federal Science Policy Office; the Fonds pour la Formation à la Recherche dans l'Industrie et dans l'Agriculture (FRIA-Belgium); the Agentschap voor Innovatie door Wetenschap en Technologie (IWT-Belgium); the F.R.S.-FNRS and FWO (Belgium) under the "Excellence of Science - EOS" - be.h project n. 30820817; the Beijing Municipal Science \& Technology Commission, No. Z191100007219010; the Ministry of Education, Youth and Sports (MEYS) of the Czech Republic; the Deutsche Forschungsgemeinschaft (DFG), under Germany's Excellence Strategy - EXC 2121 "Quantum Universe" - 390833306, and under project number 400140256 - GRK2497; the Lendület ("Momentum") Program and the János Bolyai Research Scholarship of the Hungarian Academy of Sciences, the New National Excellence Program ÚNKP, the NKFIA research grants 123842, 123959, 124845, 124850, 125105, 128713, 128786, and 129058 (Hungary); the Council of Science and Industrial Research, India; the Latvian Council of Science; the Ministry of Science and Higher Education and the National Science Center, contracts Opus 2014/15/B/ST2/03998 and 2015/19/B/ST2/02861 (Poland); the National Priorities Research Program by Qatar National Research Fund; the Ministry of Science and Higher Education, project no. 07232020-0041 (Russia); the Programa Estatal de Fomento de la Investigación Científica y Técnica de Excelencia María de Maeztu, grant MDM-2015-0509 and the Programa Severo Ochoa del Principado de Asturias; the Stavros Niarchos Foundation (Greece); the Rachadapisek Sompot Fund for Postdoctoral Fellowship, Chulalongkorn University and the Chulalongkorn Academic into Its 2nd Century Project Advancement Project (Thailand); the Kavli Foundation; the Nvidia Corporation; the SuperMicro Corporation; the Welch Foundation, contract C-1845; and the Weston Havens Foundation (U.S.A.).

Open Access. This article is distributed under the terms of the Creative Commons Attribution License (CC-BY 4.0), which permits any use, distribution and reproduction in any medium, provided the original author(s) and source are credited.

\section{References}

[1] Particle Data Group collaboration, Review of particle physics, PTEP 2020 (2020) 083C01 [INSPIRE].

[2] L. Canetti, M. Drewes and M. Shaposhnikov, Matter and antimatter in the universe, New J. Phys. 14 (2012) 095012 [arXiv: 1204.4186] [INSPIRE].

[3] K. Arun, S.B. Gudennavar and C. Sivaram, Dark matter, dark energy, and alternate models: a review, Adv. Space Res. 60 (2017) 166 [arXiv:1704.06155] [INSPIRE].

[4] W. Buchmüller and D. Wyler, Effective Lagrangian analysis of new interactions and flavor conservation, Nucl. Phys. B 268 (1986) 621 [INSPIRE].

[5] C.P. Burgess, Introduction to effective field theory, Ann. Rev. Nucl. Part. Sci. 57 (2007) 329 [hep-th/0701053] [INSPIRE].

[6] A. Helset and A. Kobach, Baryon number, lepton number, and operator dimension in the SMEFT with flavor symmetries, Phys. Lett. B 800 (2020) 135132 [arXiv:1909.05853] [INSPIRE]. 
[7] CMS collaboration, Measurement of the top quark mass using proton-proton data at $\sqrt{s}=7$ and 8 TeV, Phys. Rev. D 93 (2016) 072004 [arXiv: 1509.04044] [InSPIRE].

[8] B.A. Dobrescu and C.T. Hill, Electroweak symmetry breaking via top condensation seesaw, Phys. Rev. Lett. 81 (1998) 2634 [hep-ph/9712319] [INSPIRE].

[9] R.S. Chivukula, B.A. Dobrescu, H. Georgi and C.T. Hill, Top quark seesaw theory of electroweak symmetry breaking, Phys. Rev. D 59 (1999) 075003 [hep-ph/9809470] [INSPIRE].

[10] D. Delepine, J.M. Gerard and R. Gonzalez Felipe, Is the standard Higgs scalar elementary?, Phys. Lett. B 372 (1996) 271 [hep-ph/9512339] [INSPIRE].

[11] CMS collaboration, Observation of single top quark production in association with a $Z$ boson in proton-proton collisions at $\sqrt{s}=13 \mathrm{TeV}$, Phys. Rev. Lett. 122 (2019) 132003 [arXiv: 1812.05900] [INSPIRE].

[12] ATLAS collaboration, Observation of the associated production of a top quark and a $Z$ boson in pp collisions at $\sqrt{s}=13 \mathrm{TeV}$ with the ATLAS detector, JHEP 07 (2020) 124 [arXiv: 2002.07546] [INSPIRE].

[13] CMS collaboration, Observation of top quark pairs produced in association with a vector boson in pp collisions at $\sqrt{s}=8 \mathrm{TeV}$, JHEP 01 (2016) 096 [arXiv:1510.01131] [INSPIRE].

[14] ATLAS collaboration, Measurements of the inclusive and differential production cross sections of a top-quark-antiquark pair in association with a $Z$ boson at $\sqrt{s}=13 \mathrm{TeV}$ with the ATLAS detector, Eur. Phys. J. C 81 (2021) 737 [arXiv:2103.12603] [INSPIRE].

[15] C. Grojean, O. Matsedonskyi and G. Panico, Light top partners and precision physics, JHEP 10 (2013) 160 [arXiv:1306.4655] [INSPIRE].

[16] T. Ibrahim and P. Nath, The chromoelectric dipole moment of the top quark in models with vector like multiplets, Phys. Rev. D 84 (2011) 015003 [arXiv:1104.3851] [INSPIRE].

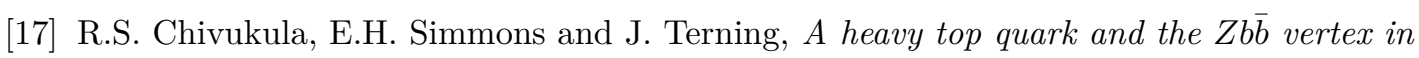
noncommuting extended technicolor, Phys. Lett. B 331 (1994) 383 [hep-ph/9404209] [INSPIRE].

[18] CMS collaboration, Measurement of the top quark polarization and $t \bar{t}$ spin correlations using dilepton final states in proton-proton collisions at $\sqrt{s}=13 \mathrm{TeV}$, Phys. Rev. D 100 (2019) 072002 [arXiv: 1907.03729] [INSPIRE].

[19] D. Barducci et al., Interpreting top-quark LHC measurements in the standard-model effective field theory, arXiv: 1802.07237 [INSPIRE].

[20] I. Brivio et al., O new physics, where art thou? A global search in the top sector, JHEP 02 (2020) 131 [arXiv:1910.03606] [INSPIRE].

[21] F. Maltoni, L. Mantani and K. Mimasu, Top-quark electroweak interactions at high energy, JHEP 10 (2019) 004 [arXiv:1904.05637] [INSPIRE].

[22] J. Brehmer, K. Cranmer, G. Louppe and J. Pavez, A guide to constraining effective field theories with machine learning, Phys. Rev. D 98 (2018) 052004 [arXiv:1805.00020] [INSPIRE].

[23] J. Hollingsworth and D. Whiteson, Resonance searches with machine learned likelihood ratios, arXiv:2002.04699 [INSPIRE].

[24] F.F. Freitas, C.K. Khosa and V. Sanz, Exploring the standard model EFT in VH production with machine learning, Phys. Rev. D 100 (2019) 035040 [arXiv:1902.05803] [INSPIRE]. 
[25] CMS collaboration, Search for new physics in top quark production with additional leptons in proton-proton collisions at $\sqrt{s}=13 \mathrm{TeV}$ using effective field theory, JHEP 03 (2021) 095 [arXiv: 2012.04120] [INSPIRE].

[26] CMS collaboration, Precision luminosity measurement in proton-proton collisions at $\sqrt{s}=13 \mathrm{TeV}$ in 2015 and 2016 at CMS, Eur. Phys. J. C 81 (2021) 800 [arXiv:2104.01927] [INSPIRE].

[27] CMS collaboration, CMS luminosity measurement for the 2017 data-taking period at $\sqrt{s}=13 \mathrm{TeV}$, Tech. Rep. CMS-PAS-LUM-17-004, CERN, Geneva, Switzerland (2018).

[28] CMS collaboration, CMS luminosity measurement for the 2018 data-taking period at $\sqrt{s}=13 \mathrm{TeV}$, Tech. Rep. CMS-PAS-LUM-18-002, CERN, Geneva, Switzerland (2019).

[29] HEPData record for this analysis, CMS-TOP-21-001, (2021).

[30] CMS collaboration, Performance of the CMS level-1 trigger in proton-proton collisions at $\sqrt{s}=13 \mathrm{TeV}, 2020$ JINST 15 P10017 [arXiv:2006.10165] [INSPIRE].

[31] CMS collaboration, The CMS trigger system, 2017 JINST 12 P01020 [arXiv:1609. 02366] [INSPIRE].

[32] CMS collaboration, The CMS experiment at the CERN LHC, 2008 JINST 3 S08004 [INSPIRE].

[33] J. Alwall et al., The automated computation of tree-level and next-to-leading order differential cross sections, and their matching to parton shower simulations, JHEP 07 (2014) 079 [arXiv: 1405.0301] [INSPIRE].

[34] J.M. Campbell, R.K. Ellis and C. Williams, Vector boson pair production at the LHC, JHEP 07 (2011) 018 [arXiv: 1105.0020] [inSPIRE].

[35] J.M. Campbell and R.K. Ellis, An update on vector boson pair production at hadron colliders, Phys. Rev. D 60 (1999) 113006 [hep-ph/9905386] [INSPIRE].

[36] P. Nason, A new method for combining NLO QCD with shower Monte Carlo algorithms, JHEP 11 (2004) 040 [hep-ph/0409146] [INSPIRE].

[37] S. Frixione, P. Nason and C. Oleari, Matching NLO QCD computations with parton shower simulations: the POWHEG method, JHEP 11 (2007) 070 [arXiv: 0709.2092] [INSPIRE].

[38] S. Alioli, P. Nason, C. Oleari and E. Re, A general framework for implementing NLO calculations in shower Monte Carlo programs: the POWHEG BOX, JHEP 06 (2010) 043 [arXiv: 1002.2581] [INSPIRE].

[39] P. Nason and G. Zanderighi, $W^{+} W^{-}, W Z$ and $Z Z$ production in the POWHEG-BOX-V2, Eur. Phys. J. C $\mathbf{7 4}$ (2014) 2702 [arXiv:1311.1365] [inSPIRE].

[40] H.B. Hartanto, B. Jäger, L. Reina and D. Wackeroth, Higgs boson production in association with top quarks in the POWHEG BOX, Phys. Rev. D 91 (2015) 094003 [arXiv:1501.04498] [INSPIRE].

[41] F. Maltoni, G. Ridolfi and M. Ubiali, b-initiated processes at the LHC: a reappraisal, JHEP 07 (2012) 022 [Erratum ibid. 04 (2013) 095] [arXiv: 1203.6393] [INSPIRE].

[42] NNPDF collaboration, Parton distributions from high-precision collider data, Eur. Phys. J. C 77 (2017) 663 [arXiv: 1706.00428] [INSPIRE].

[43] NNPDF collaboration, Parton distributions for the LHC run II, JHEP 04 (2015) 040 [arXiv: 1410.8849$]$ [INSPIRE]. 
[44] T. Sjöstrand et al., An introduction to PYTHIA 8.2, Comput. Phys. Commun. 191 (2015) 159 [arXiv: 1410.3012] [INSPIRE].

[45] CMS collaboration, Extraction and validation of a new set of CMS PYTHIA8 tunes from underlying-event measurements, Eur. Phys. J. C 80 (2020) 4 [arXiv:1903.12179] [InSPIRE].

[46] CMS collaboration, Investigations of the impact of the parton shower tuning in PYTHIA 8 in the modelling of $t \bar{t}$ at $\sqrt{s}=8$ and $13 \mathrm{TeV}$, Tech. Rep. CMS-PAS-TOP-16-021, CERN, Geneva, Switzerland (2016).

[47] CMS collaboration, Event generator tunes obtained from underlying event and multiparton scattering measurements, Eur. Phys. J. C 76 (2016) 155 [arXiv:1512.00815] [INSPIRE].

[48] R. Frederix and S. Frixione, Merging meets matching in MC@NLO, JHEP 12 (2012) 061 [arXiv: 1209.6215] [INSPIRE].

[49] J. Alwall et al., Comparative study of various algorithms for the merging of parton showers and matrix elements in hadronic collisions, Eur. Phys. J. C 53 (2008) 473 [arXiv:0706.2569] [INSPIRE].

[50] GEANT4 collaboration, GEANT4 - a simulation toolkit, Nucl. Instrum. Meth. A 506 (2003) 250 [INSPIRE].

[51] P. Artoisenet, R. Frederix, O. Mattelaer and R. Rietkerk, Automatic spin-entangled decays of heavy resonances in Monte Carlo simulations, JHEP 03 (2013) 015 [arXiv:1212.3460] [INSPIRE].

[52] B. Grzadkowski, M. Iskrzynski, M. Misiak and J. Rosiek, Dimension-six terms in the standard model Lagrangian, JHEP 10 (2010) 085 [arXiv: 1008.4884] [INSPIRE].

[53] LHC Higgs Cross Section Working Group collaboration, Handbook of LHC Higgs cross sections: 4. Deciphering the nature of the Higgs sector, arXiv:1610.07922 [INSPIRE].

[54] CMS collaboration, Measurement of the associated production of a single top quark and a $Z$ boson in pp collisions at $\sqrt{s}=13 \mathrm{TeV}$, Phys. Lett. B 779 (2018) 358 [arXiv:1712.02825] [INSPIRE].

[55] CMS collaboration, Particle-flow reconstruction and global event description with the CMS detector, 2017 JINST 12 P10003 [arXiv:1706.04965] [INSPIRE].

[56] M. Cacciari, G.P. Salam and G. Soyez, The anti- $k_{t}$ jet clustering algorithm, JHEP 04 (2008) 063 [arXiv:0802.1189] [INSPIRE].

[57] M. Cacciari, G.P. Salam and G. Soyez, FastJet user manual, Eur. Phys. J. C 72 (2012) 1896 [arXiv: 1111.6097] [INSPIRE].

[58] CMS collaboration, Performance of electron reconstruction and selection with the CMS detector in proton-proton collisions at $\sqrt{s}=8 \mathrm{TeV}, 2015$ JINST $10 \mathrm{P} 06005$ [arXiv: 1502 . 02701] [INSPIRE].

[59] CMS collaboration, Performance of the CMS muon detector and muon reconstruction with proton-proton collisions at $\sqrt{s}=13 \mathrm{TeV}, 2018$ JINST $13 \mathrm{P} 06015$ [arXiv: 1804.04528] [INSPIRE].

[60] CMS collaboration, Measurement of the Higgs boson production rate in association with top quarks in final states with electrons, muons, and hadronically decaying tau leptons at $\sqrt{s}=13 \mathrm{TeV}$, Eur. Phys. J. C 81 (2021) 378 [arXiv: 2011. 03652] [INSPIRE]. 
[61] M. Cacciari and G.P. Salam, Pileup subtraction using jet areas, Phys. Lett. B 659 (2008) 119 [arXiv: 0707.1378] [INSPIRE].

[62] CMS collaboration, Jet energy scale and resolution in the CMS experiment in pp collisions at $8 \mathrm{TeV}, 2017$ JINST 12 P02014 [arXiv:1607.03663] [INSPIRE].

[63] CMS collaboration, Identification of heavy-flavour jets with the CMS detector in pp collisions at $13 \mathrm{TeV}, 2018$ JINST 13 P05011 [arXiv: 1712.07158] [INSPIRE].

[64] E. Bols, J. Kieseler, M. Verzetti, M. Stoye and A. Stakia, Jet flavour classification using DeepJet, 2020 JINST 15 P12012 [arXiv:2008.10519] [INSPIRE].

[65] CMS collaboration, The CMS phase-1 pixel detector - experience and lessons learned from two years of operation, 2019 JINST 14 C07008 [INSPIRE].

[66] CMS collaboration, Performance of the DeepJet $b$ tagging algorithm using $41.9 \mathrm{fb}^{-1}$ of data from proton-proton collisions at $13 \mathrm{TeV}$ with phase $1 \mathrm{CMS}$ detector, Tech. Rep. CMS-DP-2018-058, CERN, Geneva, Switzerland (2018).

[67] CMS collaboration, Performance of missing transverse momentum reconstruction in proton-proton collisions at $\sqrt{s}=13 \mathrm{TeV}$ using the CMS detector, 2019 JINST $14 \mathrm{P} 07004$ [arXiv: 1903.06078] [INSPIRE].

[68] M. Abadi et al., TensorFlow: large-scale machine learning on heterogeneous distributed systems, http://tensorflow.org/, (2016).

[69] F. Chollet et al., Keras, https://github.com/fchollet/keras, (2015).

[70] D.P. Kingma and J. Ba, Adam: a method for stochastic optimization, arXiv:1412.6980 [INSPIRE].

[71] I. Goodfellow, Y. Bengio and A. Courville, Deep learning, http://www.deeplearningbook.org, MIT Press, Cambridge, MA, U.S.A. (2016).

[72] N. Srivastava et al., Dropout: a simple way to prevent neural networks from overfitting, J. Mach. Learn. Res. 15 (2014) 1929.

[73] A.N. Tikhonov, Solution of incorrectly formulated problems and the regularization method, Sov. Math. Dokl. 4 (1963) 1035.

[74] X. Glorot, A. Bordes and Y. Bengio, Deep sparse rectifier neural networks, in Proceedings, $14^{\text {th }}$ international conference on artificial intelligence and statistics (AISTATS 2011), volume 15, (2011), pg. 315.

[75] J. D'Hondt, A. Mariotti, K. Mimasu, S. Moortgat and C. Zhang, Learning to pinpoint

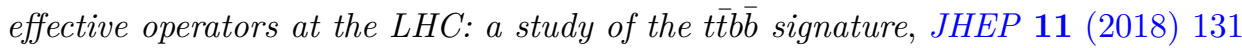
[arXiv: 1807.02130] [INSPIRE].

[76] CMS collaboration, Measurement of the inelastic proton-proton cross section at $\sqrt{s}=13 \mathrm{TeV}, \mathrm{JHEP} 07$ (2018) 161 [arXiv:1802.02613] [INSPIRE].

[77] J. Butterworth et al., PDF4LHC recommendations for LHC run II, J. Phys. G 43 (2016) 023001 [arXiv: 1510.03865] [INSPIRE].

[78] CMS collaboration, Measurements of the $p p \rightarrow W Z$ inclusive and differential production cross section and constraints on charged anomalous triple gauge couplings at $\sqrt{s}=13 \mathrm{TeV}$, JHEP 04 (2019) 122 [arXiv:1901.03428] [INSPIRE]. 
[79] CMS collaboration, Measurements of the $p p \rightarrow Z Z$ production cross section and the $Z \rightarrow 4 \ell$ branching fraction, and constraints on anomalous triple gauge couplings at $\sqrt{s}=13 \mathrm{TeV}$, Eur. Phys. J. C 78 (2018) 165 [Erratum ibid. 78 (2018) 515] [arXiv:1709.08601] [InSPIRE].

[80] CMS collaboration, Observation of the production of three massive gauge bosons at $\sqrt{s}=13 \mathrm{TeV}$, Phys. Rev. Lett. 125 (2020) 151802 [arXiv:2006.11191] [InSPIRE].

[81] CMS collaboration, Measurement of the $W \gamma$ and $Z \gamma$ inclusive cross sections in pp collisions at $\sqrt{s}=7 \mathrm{TeV}$ and limits on anomalous triple gauge boson couplings, Phys. Rev. D 89 (2014) 092005 [arXiv: 1308.6832] [INSPIRE].

[82] ATLAS collaboration, Measurements of inclusive and differential cross-sections of combined $t \bar{t} \gamma$ and $t W \gamma$ production in the e $\mu$ channel at $13 \mathrm{TeV}$ with the ATLAS detector, JHEP 09 (2020) 049 [arXiv : 2007.06946] [INSPIRE].

[83] CMS collaboration, Measurement of top quark pair production in association with a $Z$ boson in proton-proton collisions at $\sqrt{s}=13 \mathrm{TeV}$, JHEP 03 (2020) 056 [arXiv:1907.11270] [INSPIRE].

[84] R.J. Barlow and C. Beeston, Fitting using finite Monte Carlo samples, Comput. Phys. Commun. 77 (1993) 219 [INSPIRE].

[85] J.S. Conway, Incorporating nuisance parameters in likelihoods for multisource spectra, in PHYSTAT 2011, CERN-2011-006, (2011), pg. 115 [arXiv:1103.0354] [INSPIRE]. 


\section{The CMS collaboration}

\section{Deutsches Elektronen-Synchrotron, Hamburg, Germany}

N. Tonon ${ }^{\dagger}$, H. Aarup Petersen, M. Aldaya Martin, P. Asmuss, S. Baxter, M. Bayatmakou, O. Behnke, A. Bermúdez Martínez, S. Bhattacharya, A.A. Bin Anuar, K. Borras ${ }^{20}$, D. Brunner, A. Campbell, A. Cardini, C. Cheng, F. Colombina, S. Consuegra Rodríguez, G. Correia Silva, V. Danilov, M. De Silva, L. Didukh, G. Eckerlin, D. Eckstein, L.I. Estevez Banos, O. Filatov, E. Gallo ${ }^{21}$, A. Geiser, A. Giraldi, A. Grohsjean, M. Guthoff, A. Jafari ${ }^{22}$, N.Z. Jomhari, H. Jung, A. Kasem ${ }^{20}$, M. Kasemann, H. Kaveh, C. Kleinwort, D. Krücker, W. Lange, J. Lidrych, K. Lipka, W. Lohmann ${ }^{23}$, R. Mankel, I.-A. MelzerPellmann, M. Mendizabal Morentin, J. Metwally, A.B. Meyer, M. Meyer, J. Mnich, A. Mussgiller, Y. Otarid, D. Pérez Adán, D. Pitzl, A. Raspereza, B. Ribeiro Lopes, J. Rübenach, A. Saggio, A. Saibel, M. Savitskyi, M. Scham ${ }^{24}$, V. Scheurer, P. Schütze, C. Schwanenberger ${ }^{21}$, A. Singh, R.E. Sosa Ricardo, D. Stafford, M. Van De Klundert, R. Walsh, D. Walter, Y. Wen, K. Wichmann, L. Wiens, C. Wissing, S. Wuchterl

\section{Yerevan Physics Institute, Yerevan, Armenia}

A. Tumasyan

\section{Institut für Hochenergiephysik, Wien, Austria}

W. Adam, J.W. Andrejkovic, T. Bergauer, S. Chatterjee, M. Dragicevic, A. Escalante Del Valle, R. Frühwirth $^{1}$, M. Jeitler ${ }^{1}$, N. Krammer, L. Lechner, D. Liko, I. Mikulec, P. Paulitsch, F.M. Pitters, J. Schieck ${ }^{1}$, R. Schöfbeck, D. Schwarz, S. Templ, W. Waltenberger, C.-E. Wulz ${ }^{1}$

Institute for Nuclear Problems, Minsk, Belarus

V. Chekhovsky, A. Litomin, V. Makarenko

\section{Universiteit Antwerpen, Antwerpen, Belgium}

M.R. Darwish ${ }^{2}$, E.A. De Wolf, T. Janssen, T. Kello ${ }^{3}$, A. Lelek, H. Rejeb Sfar, P. Van Mechelen, S. Van Putte, N. Van Remortel

\section{Vrije Universiteit Brussel, Brussel, Belgium}

F. Blekman, E.S. Bols, J. D'Hondt, M. Delcourt, H. El Faham, S. Lowette, S. Moortgat, A. Morton, D. Müller, A.R. Sahasransu, S. Tavernier, W. Van Doninck, P. Van Mulders

\section{Université Libre de Bruxelles, Bruxelles, Belgium}

D. Beghin, B. Bilin, B. Clerbaux, G. De Lentdecker, L. Favart, A. Grebenyuk, A.K. Kalsi, K. Lee, M. Mahdavikhorrami, I. Makarenko, L. Moureaux, L. Pétré, A. Popov, N. Postiau, E. Starling, L. Thomas, M. Vanden Bemden, C. Vander Velde, P. Vanlaer, L. Wezenbeek

\section{Ghent University, Ghent, Belgium}

T. Cornelis, D. Dobur, J. Knolle, L. Lambrecht, G. Mestdach, M. Niedziela, C. Roskas, A. Samalan, K. Skovpen, M. Tytgat, B. Vermassen, M. Vit 
Université Catholique de Louvain, Louvain-la-Neuve, Belgium

A. Benecke, A. Bethani, G. Bruno, F. Bury, C. Caputo, P. David, C. Delaere, I.S. Donertas,

A. Giammanco, K. Jaffel, Sa. Jain, V. Lemaitre, K. Mondal, J. Prisciandaro, A. Taliercio, M. Teklishyn, T.T. Tran, P. Vischia, S. Wertz

Centro Brasileiro de Pesquisas Fisicas, Rio de Janeiro, Brazil

G.A. Alves, C. Hensel, A. Moraes

Universidade do Estado do Rio de Janeiro, Rio de Janeiro, Brazil

W.L. Aldá Júnior, M. Alves Gallo Pereira, M. Barroso Ferreira Filho, H. Brandao Malbouisson, W. Carvalho, J. Chinellato ${ }^{4}$, E.M. Da Costa, G.G. Da Silveira ${ }^{5}$, D. De Jesus Damiao, S. Fonseca De Souza, D. Matos Figueiredo, C. Mora Herrera, K. Mota Amarilo, L. Mundim, H. Nogima, P. Rebello Teles, A. Santoro, S.M. Silva Do Amaral, A. Sznajder, M. Thiel, F. Torres Da Silva De Araujo, A. Vilela Pereira

Universidade Estadual Paulista ${ }^{a}$, Universidade Federal do $\mathrm{ABC}^{b}$, São Paulo, Brazil

C.A. Bernardes ${ }^{a, a, 5}$, L. Calligaris ${ }^{a}$, T.R. Fernandez Perez Tomei ${ }^{a}$, E.M. Gregores ${ }^{a, b}$, D.S. Lemos ${ }^{a}$, P.G. Mercadante ${ }^{a, b}$, S.F. Novaes ${ }^{a}$, Sandra S. Padula ${ }^{a}$

Institute for Nuclear Research and Nuclear Energy, Bulgarian Academy of Sciences, Sofia, Bulgaria

A. Aleksandrov, G. Antchev, R. Hadjiiska, P. Iaydjiev, M. Misheva, M. Rodozov, M. Shopova, G. Sultanov

University of Sofia, Sofia, Bulgaria

A. Dimitrov, T. Ivanov, L. Litov, B. Pavlov, P. Petkov, A. Petrov

Beihang University, Beijing, China

T. Cheng, T. Javaid ${ }^{6}$, M. Mittal, L. Yuan

Department of Physics, Tsinghua University, Beijing, China

M. Ahmad, G. Bauer, C. Dozen ${ }^{7}$, Z. Hu, J. Martins ${ }^{8}$, Y. Wang, K. Yi ${ }^{9,10}$

Institute of High Energy Physics, Beijing, China

E. Chapon, G.M. Chen ${ }^{6}$, H.S. Chen ${ }^{6}$, M. Chen, F. Iemmi, A. Kapoor, D. Leggat, H. Liao, Z.-A. Liu ${ }^{6}$, V. Milosevic, F. Monti, R. Sharma, J. Tao, J. Thomas-Wilsker, J. Wang, H. Zhang, J. Zhao

State Key Laboratory of Nuclear Physics and Technology, Peking University, Beijing, China

A. Agapitos, Y. An, Y. Ban, C. Chen, A. Levin, Q. Li, X. Lyu, Y. Mao, S.J. Qian, D. Wang, Q. Wang, J. Xiao

Sun Yat-Sen University, Guangzhou, China

M. Lu, Z. You 
Institute of Modern Physics and Key Laboratory of Nuclear Physics and Ionbeam Application (MOE) — Fudan University, Shanghai, China

X. $\mathrm{Gao}^{3}$, H. Okawa

Zhejiang University, Hangzhou, China

Z. Lin, M. Xiao

Universidad de Los Andes, Bogota, Colombia

C. Avila, A. Cabrera, C. Florez, J. Fraga

Universidad de Antioquia, Medellin, Colombia

J. Mejia Guisao, F. Ramirez, J.D. Ruiz Alvarez, C.A. Salazar González

University of Split, Faculty of Electrical Engineering, Mechanical Engineering and Naval Architecture, Split, Croatia

D. Giljanovic, N. Godinovic, D. Lelas, I. Puljak

University of Split, Faculty of Science, Split, Croatia

Z. Antunovic, M. Kovac, T. Sculac

Institute Rudjer Boskovic, Zagreb, Croatia

V. Brigljevic, D. Ferencek, D. Majumder, M. Roguljic, A. Starodumov ${ }^{11}$, T. Susa

University of Cyprus, Nicosia, Cyprus

A. Attikis, K. Christoforou, E. Erodotou, A. Ioannou, G. Kole, M. Kolosova, S. Konstantinou, J. Mousa, C. Nicolaou, F. Ptochos, P.A. Razis, H. Rykaczewski, H. Saka

Charles University, Prague, Czech Republic

M. Finger ${ }^{12}$, M. Finger Jr. ${ }^{12}$, A. Kveton

Escuela Politecnica Nacional, Quito, Ecuador

E. Ayala

Universidad San Francisco de Quito, Quito, Ecuador

E. Carrera Jarrin

Academy of Scientific Research and Technology of the Arab Republic of Egypt, Egyptian Network of High Energy Physics, Cairo, Egypt

H. Abdalla ${ }^{13}$, Y. Assran ${ }^{14,15}$

Center for High Energy Physics (CHEP-FU), Fayoum University, El-Fayoum, Egypt

A. Lotfy, M.A. Mahmoud

National Institute of Chemical Physics and Biophysics, Tallinn, Estonia

S. Bhowmik, R.K. Dewanjee, K. Ehataht, M. Kadastik, S. Nandan, C. Nielsen, J. Pata,

M. Raidal, L. Tani, C. Veelken

Department of Physics, University of Helsinki, Helsinki, Finland

P. Eerola, L. Forthomme, H. Kirschenmann, K. Osterberg, M. Voutilainen 


\section{Helsinki Institute of Physics, Helsinki, Finland}

S. Bharthuar, E. Brücken, F. Garcia, J. Havukainen, M.S. Kim, R. Kinnunen, T. Lampén, K. Lassila-Perini, S. Lehti, T. Lindén, M. Lotti, L. Martikainen, M. Myllymäki, J. Ott, H. Siikonen, E. Tuominen, J. Tuominiemi

Lappeenranta University of Technology, Lappeenranta, Finland P. Luukka, H. Petrow, T. Tuuva

IRFU, CEA, Université Paris-Saclay, Gif-sur-Yvette, France

C. Amendola, M. Besancon, F. Couderc, M. Dejardin, D. Denegri, J.L. Faure, F. Ferri, S. Ganjour, A. Givernaud, P. Gras, G. Hamel de Monchenault, P. Jarry, B. Lenzi, E. Locci, J. Malcles, J. Rander, A. Rosowsky, M.Ö. Sahin, A. Savoy-Navarro ${ }^{16}$, M. Titov, G.B. Yu

Laboratoire Leprince-Ringuet, CNRS/IN2P3, Ecole Polytechnique, Institut Polytechnique de Paris, Palaiseau, France

S. Ahuja, F. Beaudette, M. Bonanomi, A. Buchot Perraguin, P. Busson, A. Cappati, C. Charlot, O. Davignon, B. Diab, G. Falmagne, S. Ghosh, R. Granier de Cassagnac,

A. Hakimi, I. Kucher, J. Motta, M. Nguyen, C. Ochando, P. Paganini, J. Rembser, R. Salerno, U. Sarkar, J.B. Sauvan, Y. Sirois, A. Tarabini, A. Zabi, A. Zghiche

Université de Strasbourg, CNRS, IPHC UMR 7178, Strasbourg, France J.-L. Agram ${ }^{17}$, J. Andrea, D. Apparu, D. Bloch, G. Bourgatte, J.-M. Brom, E.C. Chabert, C. Collard, D. Darej, J.-C. Fontaine ${ }^{17}$, U. Goerlach, C. Grimault, A.-C. Le Bihan, E. Nibigira, P. Van Hove

Institut de Physique des 2 Infinis de Lyon (IP2I ), Villeurbanne, France E. Asilar, S. Beauceron, C. Bernet, G. Boudoul, C. Camen, A. Carle, N. Chanon, D. Contardo, P. Depasse, H. El Mamouni, J. Fay, S. Gascon, M. Gouzevitch, B. Ille, I.B. Laktineh, H. Lattaud, A. Lesauvage, M. Lethuillier, L. Mirabito, S. Perries, K. Shchablo, V. Sordini, L. Torterotot, G. Touquet, M. Vander Donckt, S. Viret

Georgian Technical University, Tbilisi, Georgia

G. Adamov, I. Lomidze, Z. Tsamalaidze ${ }^{12}$

RWTH Aachen University, I. Physikalisches Institut, Aachen, Germany

V. Botta, L. Feld, K. Klein, M. Lipinski, D. Meuser, A. Pauls, N. Röwert, J. Schulz, M. Teroerde

RWTH Aachen University, III. Physikalisches Institut A, Aachen, Germany A. Dodonova, D. Eliseev, M. Erdmann, P. Fackeldey, B. Fischer, S. Ghosh, T. Hebbeker, K. Hoepfner, F. Ivone, L. Mastrolorenzo, M. Merschmeyer, A. Meyer, G. Mocellin, S. Mondal, S. Mukherjee, D. Noll, A. Novak, T. Pook, A. Pozdnyakov, Y. Rath, H. Reithler, J. Roemer, A. Schmidt, S.C. Schuler, A. Sharma, L. Vigilante, S. Wiedenbeck, S. Zaleski

RWTH Aachen University, III. Physikalisches Institut B, Aachen, Germany

C. Dziwok, G. Flügge, W. Haj Ahmad ${ }^{18}$, O. Hlushchenko, T. Kress, A. Nowack, C. Pistone, O. Pooth, D. Roy, H. Sert, A. Stahl ${ }^{19}$, T. Ziemons, A. Zotz 


\section{University of Hamburg, Hamburg, Germany}

R. Aggleton, S. Albrecht, S. Bein, L. Benato, P. Connor, K. De Leo, M. Eich, F. Feindt, A. Fröhlich, C. Garbers, E. Garutti, P. Gunnellini, M. Hajheidari, J. Haller, A. Hinzmann, G. Kasieczka, R. Klanner, R. Kogler, T. Kramer, V. Kutzner, J. Lange, T. Lange, A. Lobanov, A. Malara, A. Nigamova, K.J. Pena Rodriguez, O. Rieger, P. Schleper, M. Schröder, J. Schwandt, J. Sonneveld, H. Stadie, G. Steinbrück, A. Tews, I. Zoi

Karlsruher Institut fuer Technologie, Karlsruhe, Germany

J. Bechtel, S. Brommer, E. Butz, R. Caspart, T. Chwalek, W. De Boer ${ }^{\dagger}$, A. Dierlamm, A. Droll, K. El Morabit, N. Faltermann, M. Giffels, J.O. Gosewisch, A. Gottmann, F. Hartmann ${ }^{19}$, C. Heidecker, U. Husemann, P. Keicher, R. Koppenhöfer, S. Maier, M. Metzler, S. Mitra, Th. Müller, M. Neukum, A. Nürnberg, G. Quast, K. Rabbertz, J. Rauser, D. Savoiu, M. Schnepf, D. Seith, I. Shvetsov, H.J. Simonis, R. Ulrich, J. Van Der Linden, R.F. Von Cube, M. Wassmer, M. Weber, S. Wieland, R. Wolf, S. Wozniewski, S. Wunsch

Institute of Nuclear and Particle Physics (INPP), NCSR Demokritos, Aghia Paraskevi, Greece

G. Anagnostou, G. Daskalakis, T. Geralis, A. Kyriakis, D. Loukas, A. Stakia

National and Kapodistrian University of Athens, Athens, Greece

M. Diamantopoulou, D. Karasavvas, G. Karathanasis, P. Kontaxakis, C.K. Koraka, A. Manousakis-Katsikakis, A. Panagiotou, I. Papavergou, N. Saoulidou, K. Theofilatos, E. Tziaferi, K. Vellidis, E. Vourliotis

National Technical University of Athens, Athens, Greece

G. Bakas, K. Kousouris, I. Papakrivopoulos, G. Tsipolitis, A. Zacharopoulou

University of Ioánnina, Ioánnina, Greece

K. Adamidis, I. Bestintzanos, I. Evangelou, C. Foudas, P. Gianneios, P. Katsoulis, P. Kokkas, N. Manthos, I. Papadopoulos, J. Strologas

MTA-ELTE Lendület CMS Particle and Nuclear Physics Group, Eötvös Loránd University, Budapest, Hungary

M. Csanad, K. Farkas, M.M.A. Gadallah ${ }^{25}$, S. Lökös ${ }^{26}$, P. Major, K. Mandal, A. Mehta, G. Pasztor, A.J. Rádl, O. Surányi, G.I. Veres

Wigner Research Centre for Physics, Budapest, Hungary

M. Bartók ${ }^{27}$, G. Bencze, C. Hajdu, D. Horvath ${ }^{28}$, F. Sikler, V. Veszpremi, G. Vesztergombi ${ }^{\dagger}$

Institute of Nuclear Research ATOMKI, Debrecen, Hungary

S. Czellar, J. Karancsi ${ }^{27}$, J. Molnar, Z. Szillasi, D. Teyssier

Institute of Physics, University of Debrecen, Debrecen, Hungary

P. Raics, Z.L. Trocsanyi ${ }^{29}$, B. Ujvari

Karoly Robert Campus, MATE Institute of Technology

T. Csorgo ${ }^{30}$, F. Nemes ${ }^{30}$, T. Novak 
Indian Institute of Science (IISc), Bangalore, India

J.R. Komaragiri, D. Kumar, L. Panwar, P.C. Tiwari

National Institute of Science Education and Research, HBNI, Bhubaneswar, India

S. Bahinipati ${ }^{31}$, C. Kar, P. Mal, T. Mishra, V.K. Muraleedharan Nair Bindhu ${ }^{32}$, A. Nayak ${ }^{32}$, P. Saha, N. Sur, S.K. Swain, D. Vats ${ }^{32}$

Panjab University, Chandigarh, India

S. Bansal, S.B. Beri, V. Bhatnagar, G. Chaudhary, S. Chauhan, N. Dhingra ${ }^{33}$, R. Gupta, A. Kaur, M. Kaur, S. Kaur, P. Kumari, M. Meena, K. Sandeep, J.B. Singh, A.K. Virdi

University of Delhi, Delhi, India

A. Ahmed, A. Bhardwaj, B.C. Choudhary, M. Gola, S. Keshri, A. Kumar, M. Naimuddin, P. Priyanka, K. Ranjan, A. Shah

Saha Institute of Nuclear Physics, HBNI, Kolkata, India

M. Bharti ${ }^{34}$, R. Bhattacharya, S. Bhattacharya, D. Bhowmik, S. Dutta, S. Dutta, B. Gomber ${ }^{35}$, M. Maity ${ }^{36}$, P. Palit, P.K. Rout, G. Saha, B. Sahu, S. Sarkar, M. Sharan, B. Singh ${ }^{34}$, S. Thakur ${ }^{34}$

Indian Institute of Technology Madras, Madras, India

P.K. Behera, S.C. Behera, P. Kalbhor, A. Muhammad, R. Pradhan, P.R. Pujahari, A. Sharma, A.K. Sikdar

Bhabha Atomic Research Centre, Mumbai, India

D. Dutta, V. Jha, V. Kumar, D.K. Mishra, K. Naskar ${ }^{37}$, P.K. Netrakanti, L.M. Pant, P. Shukla

Tata Institute of Fundamental Research-A, Mumbai, India

T. Aziz, S. Dugad, M. Kumar

Tata Institute of Fundamental Research-B, Mumbai, India

S. Banerjee, R. Chudasama, M. Guchait, S. Karmakar, S. Kumar, G. Majumder, K. Mazumdar, S. Mukherjee

Indian Institute of Science Education and Research (IISER), Pune, India K. Alpana, S. Dube, B. Kansal, A. Laha, S. Pandey, A. Rane, A. Rastogi, S. Sharma

Department of Physics, Isfahan University of Technology, Isfahan, Iran

H. Bakhshiansohi ${ }^{38}$, E. Khazaie, M. Zeinali ${ }^{39}$

Institute for Research in Fundamental Sciences (IPM), Tehran, Iran

S. Chenarani ${ }^{40}$, S.M. Etesami, M. Khakzad, M. Mohammadi Najafabadi

University College Dublin, Dublin, Ireland

M. Grunewald 
INFN Sezione di Bari ${ }^{a}$, Università di Bari ${ }^{b}$, Politecnico di Bari ${ }^{c}$, Bari, Italy

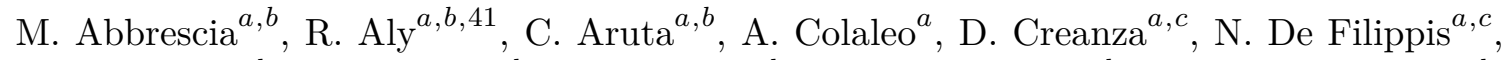
M. De Palma ${ }^{a, b}$, A. Di Florio ${ }^{a, b}$, A. Di Pilato ${ }^{a, b}$, W. Elmetenawee ${ }^{a, b}{\text {, L. } \text { Fiore }^{a} \text {, A. Gelmi }}^{a, b}$,

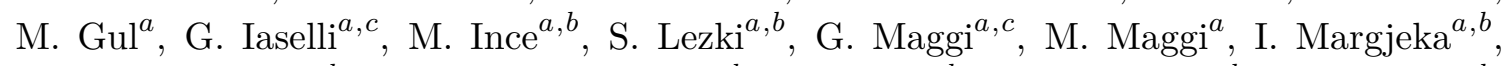
V. Mastrapasqua ${ }^{a, b}$, J.A. Merlin ${ }^{a}$, S. My ${ }^{a, b}$, S. Nuzzo ${ }^{a, b}$, A. Pellecchia ${ }^{a, b}$, A. Pompili ${ }^{a, b}$, G. Pugliese ${ }^{a, c}$, D. Ramos, A. Ranieri ${ }^{a}$, G. Selvaggi ${ }^{a, b}$, L. Silvestris ${ }^{a}$, F.M. Simone ${ }^{a, b}$, R. Venditti ${ }^{a}$, P. Verwilligen ${ }^{a}$

INFN Sezione di Bologna ${ }^{a}$, Università di Bologna ${ }^{b}$, Bologna, Italy

G. Abbiendi ${ }^{a}$, C. Battilana ${ }^{a, b}$, D. Bonacorsi ${ }^{a, b}$, L. Borgonovi ${ }^{a}$, L. Brigliadori ${ }^{a}$, R. Campanini ${ }^{a, b}$, P. Capiluppi ${ }^{a, b}$, A. Castro ${ }^{a, b}$, F.R. Cavallo ${ }^{a}$, M. Cuffiani ${ }^{a, b}$, G.M. Dallavalle ${ }^{a}$, T. Diotalevi ${ }^{a, b}$, F. Fabbri ${ }^{a}$, A. Fanfani ${ }^{a, b}$, P. Giacomelli $^{a}$, L. Giommi $^{a, b}{ }^{,}$ C. Grandi ${ }^{a}$, L. Guiducci ${ }^{a, b}$, S. Lo Meo ${ }^{a, 42}$, L. Lunerti ${ }^{a, b}$, S. Marcellini ${ }^{a}$, G. Masetti ${ }^{a}$, F.L. Navarria ${ }^{a, b}$, A. Perrotta ${ }^{a}$, F. Primavera ${ }^{a, b}$, A.M. Rossi ${ }^{a, b}$, T. Rovelli ${ }^{a, b}$, G.P. Siroli ${ }^{a, b}$

INFN Sezione di Catania $^{a}$, Università di Catania ${ }^{b}$, Catania, Italy

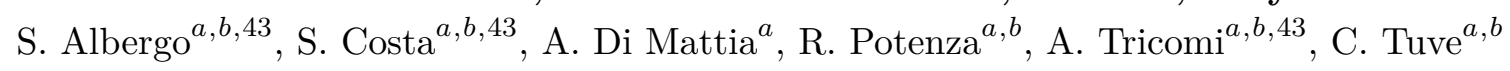
INFN Sezione di Firenze ${ }^{a}$, Università di Firenze ${ }^{b}$, Firenze, Italy

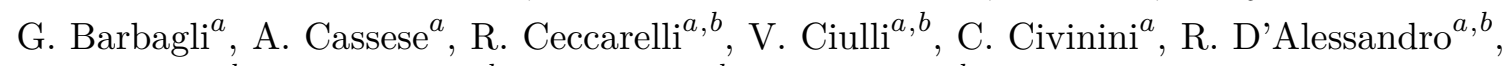
E. Focardi ${ }^{a, b}$, G. Latino ${ }^{a, b}$, P. Lenzi ${ }^{a, b}$, M. Lizzo ${ }^{a, b}$, M. Meschini ${ }^{a}$, S. Paoletti ${ }^{a}$, R. Seidita ${ }^{a, b}$, G. Sguazzoni ${ }^{a}$, L. Viliani $^{a}$

INFN Laboratori Nazionali di Frascati, Frascati, Italy

L. Benussi, S. Bianco, D. Piccolo

INFN Sezione di Genova ${ }^{a}$, Università di Genova ${ }^{b}$, Genova, Italy

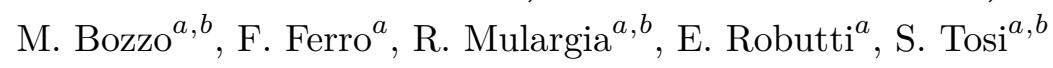

INFN Sezione di Milano-Bicocca ${ }^{a}$, Università di Milano-Bicocca ${ }^{b}$, Milano, Italy

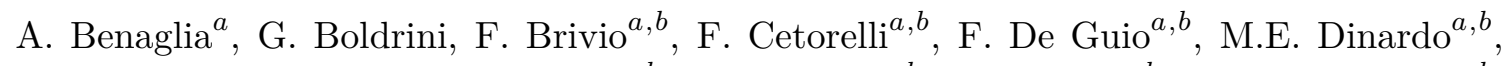
P. Dini ${ }^{a}$, S. Gennai ${ }^{a}$, A. Ghezzi ${ }^{a, b}$, P. Govoni ${ }^{a, b}$, L. Guzzi ${ }^{a, b}$, M.T. Lucchini ${ }^{a, b}$, M. Malberti ${ }^{a}$, S. Malvezzi ${ }^{a}$, A. Massironi ${ }^{a}$, D. Menasce ${ }^{a}$, L. Moroni ${ }^{a}$, M. Paganoni ${ }^{a, b}$, D. Pedrini ${ }^{a}$, B.S. Pinolini, S. Ragazzi ${ }^{a, b}$, N. Redaelli ${ }^{a}$, T. Tabarelli de Fatis ${ }^{a, b}$, D. Valsecchi ${ }^{a, b, 19}$, D. Zuolo ${ }^{a, b}$

INFN Sezione di Napoli ${ }^{a}$, Università di Napoli 'Federico II' ${ }^{b}$, Napoli, Italy, Università della Basilicata ${ }^{c}$, Potenza, Italy, Università G. Marconi ${ }^{d}$, Roma, Italy

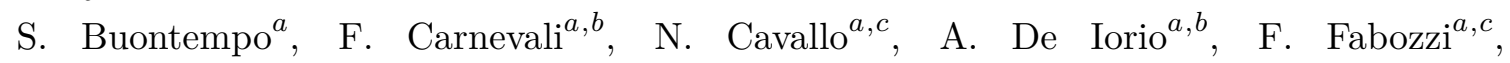

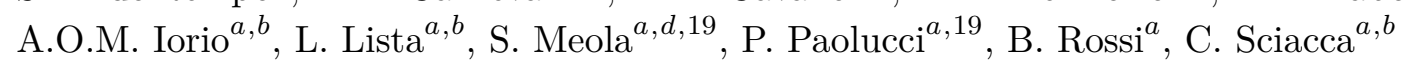

INFN Sezione di Padova ${ }^{a}$, Università di Padova ${ }^{b}$, Padova, Italy, Università di Trento ${ }^{c}$, Trento, Italy

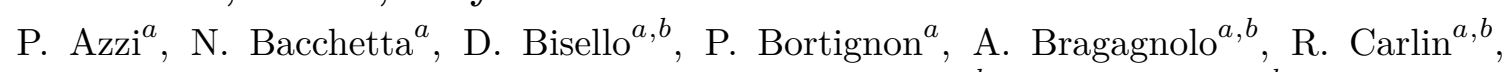
P. Checchia ${ }^{a}$, T. Dorigo ${ }^{a}$, U. Dosselli ${ }^{a}$, F. Gasparini ${ }^{a, b}$, U. Gasparini ${ }^{a, b}$, G. Grosso, 


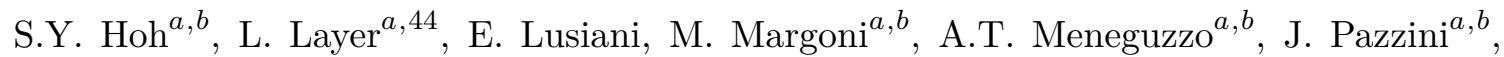

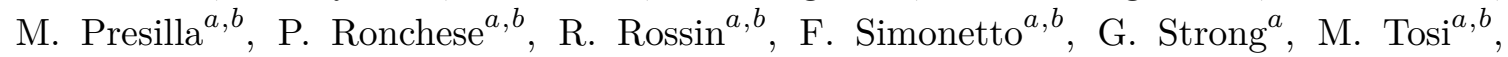

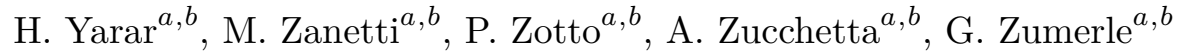

INFN Sezione di Pavia ${ }^{a}$, Università di Pavia ${ }^{b}$, Pavia, Italy

C. Aime ${ }^{a, b}$, A. Braghieri ${ }^{a}$, S. Calzaferri ${ }^{a, b}$, D. Fiorina ${ }^{a, b}$, P. Montagna ${ }^{a, b}$, S.P. Ratti ${ }^{a, b}$,

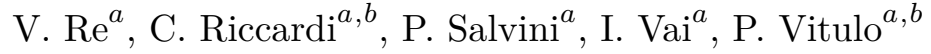

INFN Sezione di Perugia ${ }^{a}$, Università di Perugia ${ }^{b}$, Perugia, Italy

P. Asenov ${ }^{a, 45}$, G.M. Bilei ${ }^{a}$, D. Ciangottini ${ }^{a, b}$, L. Fanò ${ }^{a, b}$, P. Lariccia $^{a, b}$, M. Magherini $^{b}$,

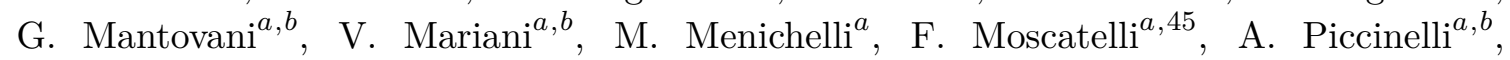

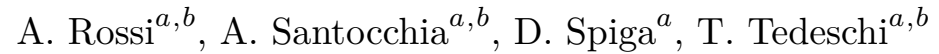

INFN Sezione di Pisa ${ }^{a}$, Università di Pisa ${ }^{b}$, Scuola Normale Superiore di Pisa ${ }^{c}$, Pisa Italy, Università di Siena ${ }^{d}$, Siena, Italy

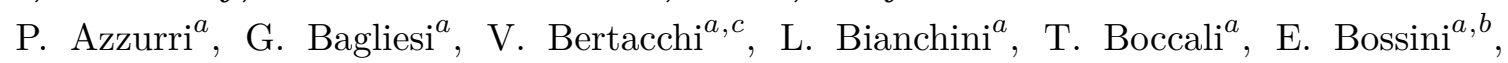

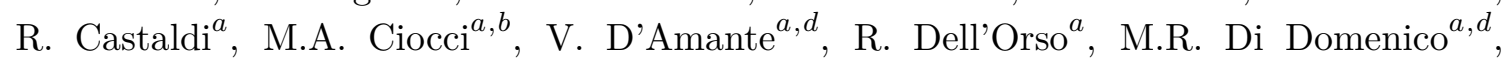

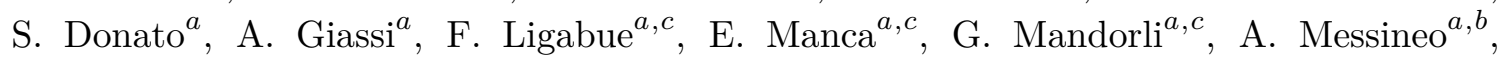
F. Palla ${ }^{a}$, S. Parolia ${ }^{a, b}$, G. Ramirez-Sanchez ${ }^{a, c}$, A. Rizzi ${ }^{a, b}$, G. Rolandi ${ }^{a, c}$, S. Roy Chowdhury ${ }^{a, c}$, A. Scribano $^{a}$, N. Shafiei ${ }^{a, b}$, P. Spagnolo $^{a}$, R. Tenchini ${ }^{a}$, G. Tonelli $^{a, b}{ }^{,}$ N. Turini ${ }^{a, d}$, A. Venturi $^{a}$, P.G. Verdini ${ }^{a}$

INFN Sezione di Roma ${ }^{a}$, Sapienza Università di Roma ${ }^{b}$, Rome, Italy

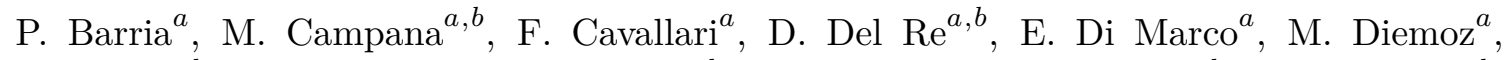

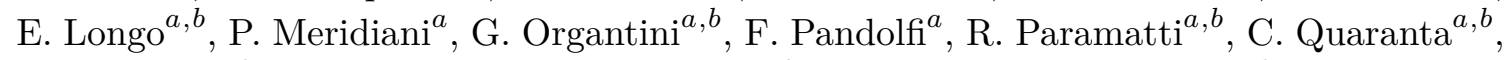

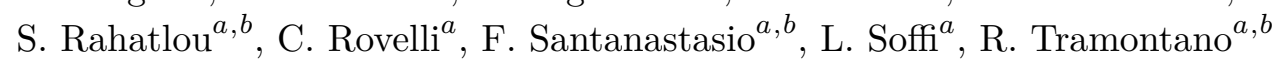

INFN Sezione di Torino ${ }^{a}$, Università di Torino ${ }^{b}$, Torino, Italy, Università del Piemonte Orientale ${ }^{c}$, Novara, Italy

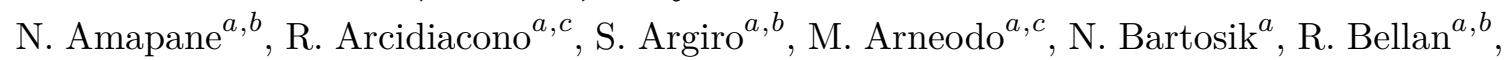
A. Bellora ${ }^{a, b}, \mathrm{~J}$ Berenguer Antequera ${ }^{a, b}$, C. Biino ${ }^{a}$, N. Cartiglia $^{a}$, S. Cometti $^{a}$, M. Costa $^{a, b}$, R. Covarelli ${ }^{a, b}$, N. Demaria ${ }^{a}$, B. Kiani ${ }^{a, b}$, F. Legger ${ }^{a}$, C. Mariotti ${ }^{a}$, S. Maselli ${ }^{a}$, E. Migliore $^{a, b}$, E. Monteil ${ }^{a, b}$, M. Monteno ${ }^{a}$, M.M. Obertino ${ }^{a, b}$, G. Ortona ${ }^{a}$, L. Pacher ${ }^{a, b}$, N. Pastrone $^{a}$, M. Pelliccioni ${ }^{a}$, G.L. Pinna Angioni ${ }^{a, b}$, M. Ruspa ${ }^{a, c}$, K. Shchelina ${ }^{a}$,

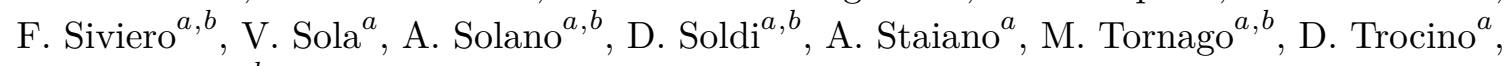
A. Vagnerini ${ }^{a, b}$

INFN Sezione di Trieste ${ }^{a}$, Università di Trieste ${ }^{b}$, Trieste, Italy

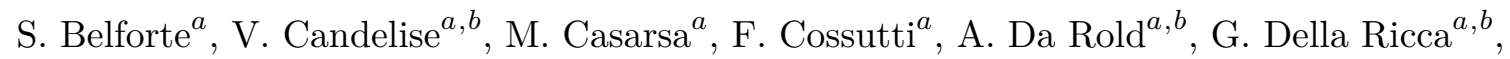
G. Sorrentino $^{a, b}$, F. Vazzoler ${ }^{a, b}$

\section{Kyungpook National University, Daegu, Korea}

S. Dogra, C. Huh, B. Kim, D.H. Kim, G.N. Kim, J. Kim, J. Lee, S.W. Lee, C.S. Moon, Y.D. Oh, S.I. Pak, B.C. Radburn-Smith, S. Sekmen, Y.C. Yang

Chonnam National University, Institute for Universe and Elementary Particles, Kwangju, Korea

H. Kim, D.H. Moon 
Hanyang University, Seoul, Korea

B. Francois, T.J. Kim, J. Park

Korea University, Seoul, Korea

S. Cho, S. Choi, Y. Go, B. Hong, K. Lee, K.S. Lee, J. Lim, J. Park, S.K. Park, J. Yoo

Kyung Hee University, Department of Physics, Seoul, Republic of Korea

J. Goh, A. Gurtu

Sejong University, Seoul, Korea

H.S. Kim, Y. Kim

Seoul National University, Seoul, Korea

J. Almond, J.H. Bhyun, J. Choi, S. Jeon, J. Kim, J.S. Kim, S. Ko, H. Kwon, H. Lee, S. Lee, B.H. Oh, M. Oh, S.B. Oh, H. Seo, U.K. Yang, I. Yoon

University of Seoul, Seoul, Korea

W. Jang, D.Y. Kang, Y. Kang, S. Kim, B. Ko, J.S.H. Lee, Y. Lee, I.C. Park, Y. Roh, M.S. Ryu, D. Song, I.J. Watson, S. Yang

Yonsei University, Department of Physics, Seoul, Korea

S. Ha, H.D. Yoo

Sungkyunkwan University, Suwon, Korea

M. Choi, H. Lee, Y. Lee, I. Yu

College of Engineering and Technology, American University of the Middle East (AUM), Egaila, Kuwait

T. Beyrouthy, Y. Maghrbi

Riga Technical University, Riga, Latvia

T. Torims, V. Veckalns ${ }^{46}$

Vilnius University, Vilnius, Lithuania

M. Ambrozas, A. Carvalho Antunes De Oliveira, A. Juodagalvis, A. Rinkevicius, G. Tamulaitis

National Centre for Particle Physics, Universiti Malaya, Kuala Lumpur, Malaysia

N. Bin Norjoharuddeen, W.A.T. Wan Abdullah, M.N. Yusli, Z. Zolkapli

Universidad de Sonora (UNISON), Hermosillo, Mexico

J.F. Benitez, A. Castaneda Hernandez, M. León Coello, J.A. Murillo Quijada, A. Sehrawat, L. Valencia Palomo

Centro de Investigacion y de Estudios Avanzados del IPN, Mexico City, Mexico G. Ayala, H. Castilla-Valdez, E. De La Cruz-Burelo, I. Heredia-De La Cruz ${ }^{47}$, R. LopezFernandez, C.A. Mondragon Herrera, D.A. Perez Navarro, A. Sanchez-Hernandez

Universidad Iberoamericana, Mexico City, Mexico

S. Carrillo Moreno, C. Oropeza Barrera, F. Vazquez Valencia 
Benemerita Universidad Autonoma de Puebla, Puebla, Mexico

I. Pedraza, H.A. Salazar Ibarguen, C. Uribe Estrada

University of Montenegro, Podgorica, Montenegro

J. Mijuskovic ${ }^{48}$, N. Raicevic

University of Auckland, Auckland, New Zealand

D. Krofcheck

University of Canterbury, Christchurch, New Zealand

P.H. Butler

National Centre for Physics, Quaid-I-Azam University, Islamabad, Pakistan

A. Ahmad, M.I. Asghar, A. Awais, M.I.M. Awan, H.R. Hoorani, W.A. Khan, M.A. Shah, M. Shoaib, M. Waqas

AGH University of Science and Technology Faculty of Computer Science, Electronics and Telecommunications, Krakow, Poland

V. Avati, L. Grzanka, M. Malawski

National Centre for Nuclear Research, Swierk, Poland

H. Bialkowska, M. Bluj, B. Boimska, M. Górski, M. Kazana, M. Szleper, P. Zalewski

Institute of Experimental Physics, Faculty of Physics, University of Warsaw, Warsaw, Poland

K. Bunkowski, K. Doroba, A. Kalinowski, M. Konecki, J. Krolikowski, M. Walczak

Laboratório de Instrumentação e Física Experimental de Partículas, Lisboa, Portugal

M. Araujo, P. Bargassa, D. Bastos, A. Boletti, P. Faccioli, M. Gallinaro, J. Hollar, N. Leonardo, T. Niknejad, M. Pisano, J. Seixas, O. Toldaiev, J. Varela

Joint Institute for Nuclear Research, Dubna, Russia

S. Afanasiev, D. Budkouski, I. Golutvin, I. Gorbunov, V. Karjavine, V. Korenkov, A. Lanev, A. Malakhov, V. Matveev ${ }^{49,50}$, V. Palichik, V. Perelygin, M. Savina, D. Seitova, V. Shalaev, S. Shmatov, S. Shulha, V. Smirnov, O. Teryaev, N. Voytishin, B.S. Yuldashev ${ }^{51}$, A. Zarubin, I. Zhizhin

Petersburg Nuclear Physics Institute, Gatchina (St. Petersburg), Russia

G. Gavrilov, V. Golovtcov, Y. Ivanov, V. Kim ${ }^{52}$, E. Kuznetsova ${ }^{53}$, V. Murzin, V. Oreshkin, I. Smirnov, D. Sosnov, V. Sulimov, L. Uvarov, S. Volkov, A. Vorobyev

Institute for Nuclear Research, Moscow, Russia

Yu. Andreev, A. Dermenev, S. Gninenko, N. Golubev, A. Karneyeu, D. Kirpichnikov, M. Kirsanov, N. Krasnikov, A. Pashenkov, G. Pivovarov, A. Toropin

Institute for Theoretical and Experimental Physics named by A.I. Alikhanov of NRC 'Kurchatov Institute', Moscow, Russia

V. Epshteyn, V. Gavrilov, N. Lychkovskaya, A. Nikitenko ${ }^{54}$, V. Popov, A. Stepennov, M. Toms, E. Vlasov, A. Zhokin 
Moscow Institute of Physics and Technology, Moscow, Russia

T. Aushev

National Research Nuclear University 'Moscow Engineering Physics Institute' (MEPhI), Moscow, Russia

M. Chadeeva ${ }^{55}$, A. Oskin, P. Parygin, E. Popova, V. Rusinov, D. Selivanova

P.N. Lebedev Physical Institute, Moscow, Russia

V. Andreev, M. Azarkin, I. Dremin, M. Kirakosyan, A. Terkulov

Skobeltsyn Institute of Nuclear Physics, Lomonosov Moscow State University, Moscow, Russia

A. Belyaev, E. Boos, V. Bunichev, M. Dubinin ${ }^{56}$, L. Dudko, A. Gribushin, V. Klyukhin,

N. Korneeva, I. Lokhtin, S. Obraztsov, M. Perfilov, V. Savrin, P. Volkov

Novosibirsk State University (NSU), Novosibirsk, Russia

V. Blinov ${ }^{57}$, T. Dimova ${ }^{57}$, L. Kardapoltsev ${ }^{57}$, A. Kozyrev ${ }^{57}$, I. Ovtin ${ }^{57}$, Y. Skovpen ${ }^{57}$

Institute for High Energy Physics of National Research Centre 'Kurchatov Institute', Protvino, Russia

I. Azhgirey, I. Bayshev, D. Elumakhov, V. Kachanov, D. Konstantinov, P. Mandrik,

V. Petrov, R. Ryutin, S. Slabospitskii, A. Sobol, S. Troshin, N. Tyurin, A. Uzunian,

A. Volkov

National Research Tomsk Polytechnic University, Tomsk, Russia

A. Babaev, V. Okhotnikov

Tomsk State University, Tomsk, Russia

V. Borshch, V. Ivanchenko, E. Tcherniaev

University of Belgrade: Faculty of Physics and VINCA Institute of Nuclear Sciences, Belgrade, Serbia

P. Adzic ${ }^{58}$, M. Dordevic, P. Milenovic, J. Milosevic

Centro de Investigaciones Energéticas Medioambientales y Tecnológicas (CIEMAT), Madrid, Spain

M. Aguilar-Benitez, J. Alcaraz Maestre, A. Álvarez Fernández, I. Bachiller, M. Barrio Luna, Cristina F. Bedoya, C.A. Carrillo Montoya, M. Cepeda, M. Cerrada, N. Colino, B. De La Cruz, A. Delgado Peris, J.P. Fernández Ramos, J. Flix, M.C. Fouz, O. Gonzalez Lopez, S. Goy Lopez, J.M. Hernandez, M.I. Josa, J. León Holgado, D. Moran, Á. Navarro Tobar, C. Perez Dengra, A. Pérez-Calero Yzquierdo, J. Puerta Pelayo, I. Redondo, L. Romero, S. Sánchez Navas, L. Urda Gómez, C. Willmott

Universidad Autónoma de Madrid, Madrid, Spain

J.F. de Trocóniz, R. Reyes-Almanza 
Universidad de Oviedo, Instituto Universitario de Ciencias y Tecnologías Espaciales de Asturias (ICTEA), Oviedo, Spain

B. Alvarez Gonzalez, J. Cuevas, C. Erice, J. Fernandez Menendez, S. Folgueras, I. Gonzalez Caballero, J.R. González Fernández, E. Palencia Cortezon, C. Ramón Álvarez, V. Rodríguez Bouza, A. Soto Rodríguez, A. Trapote, N. Trevisani, C. Vico Villalba

Instituto de Física de Cantabria (IFCA), CSIC-Universidad de Cantabria, Santander, Spain

J.A. Brochero Cifuentes, I.J. Cabrillo, A. Calderon, J. Duarte Campderros, M. Fernandez, C. Fernandez Madrazo, P.J. Fernández Manteca, A. García Alonso, G. Gomez, C. Martinez Rivero, P. Martinez Ruiz del Arbol, F. Matorras, Pablo Matorras-Cuevas,

J. Piedra Gomez, C. Prieels, T. Rodrigo, A. Ruiz-Jimeno, L. Scodellaro, I. Vila, J.M. Vizan Garcia

\section{University of Colombo, Colombo, Sri Lanka}

M.K. Jayananda, B. Kailasapathy ${ }^{59}$, D.U.J. Sonnadara, D.D.C. Wickramarathna

University of Ruhuna, Department of Physics, Matara, Sri Lanka

W.G.D. Dharmaratna, K. Liyanage, N. Perera, N. Wickramage

CERN, European Organization for Nuclear Research, Geneva, Switzerland

T.K. Aarrestad, D. Abbaneo, J. Alimena, E. Auffray, G. Auzinger, J. Baechler, P. Baillon ${ }^{\dagger}$,

D. Barney, J. Bendavid, M. Bianco, A. Bocci, T. Camporesi, M. Capeans Garrido,

G. Cerminara, S.S. Chhibra, M. Cipriani, L. Cristella, D. d'Enterria, A. Dabrowski,

A. David, A. De Roeck, M.M. Defranchis, M. Deile, M. Dobson, M. Dünser, N. Dupont,

A. Elliott-Peisert, N. Emriskova, F. Fallavollita ${ }^{60}$, D. Fasanella, A. Florent, G. Franzoni,

W. Funk, S. Giani, D. Gigi, K. Gill, F. Glege, L. Gouskos, M. Haranko, J. Hegeman,

V. Innocente, T. James, P. Janot, J. Kieseler, M. Komm, N. Kratochwil, C. Lange,

S. Laurila, P. Lecoq, A. Lintuluoto, K. Long, C. Lourenço, B. Maier, L. Malgeri, S. Mallios, M. Mannelli, A.C. Marini, F. Meijers, S. Mersi, E. Meschi, F. Moortgat, M. Mulders, S. Orfanelli, L. Orsini, F. Pantaleo, L. Pape, E. Perez, M. Peruzzi, A. Petrilli, G. Petrucciani, A. Pfeiffer, M. Pierini, D. Piparo, M. Pitt, H. Qu, T. Quast, D. Rabady, A. Racz, G. Reales Gutiérrez, M. Rieger, M. Rovere, H. Sakulin, J. Salfeld-Nebgen, S. Scarfi, C. Schäfer, C. Schwick, M. Selvaggi, A. Sharma, P. Silva, W. Snoeys, P. Sphicas ${ }^{61}$, S. Summers, K. Tatar, V.R. Tavolaro, D. Treille, P. Tropea, A. Tsirou, G.P. Van Onsem, J. Wanczyk ${ }^{62}$, K.A. Wozniak, W.D. Zeuner

Paul Scherrer Institut, Villigen, Switzerland

L. Caminada ${ }^{63}$, A. Ebrahimi, W. Erdmann, R. Horisberger, Q. Ingram, H.C. Kaestli, D. Kotlinski, U. Langenegger, M. Missiroli, L. Noehte, T. Rohe

ETH Zurich - Institute for Particle Physics and Astrophysics (IPA), Zurich, Switzerland

K. Androsov ${ }^{62}$, M. Backhaus, P. Berger, A. Calandri, N. Chernyavskaya, A. De Cosa, G. Dissertori, M. Dittmar, M. Donegà, C. Dorfer, F. Eble, K. Gedia, F. Glessgen, T.A. Gómez Espinosa, C. Grab, D. Hits, W. Lustermann, A.-M. Lyon, R.A. Manzoni, 
L. Marchese, C. Martin Perez, M.T. Meinhard, F. Nessi-Tedaldi, J. Niedziela, F. Pauss, V. Perovic, S. Pigazzini, M.G. Ratti, M. Reichmann, C. Reissel, T. Reitenspiess, B. Ristic, D. Ruini, D.A. Sanz Becerra, V. Stampf, J. Steggemann ${ }^{62}$, R. Wallny, D.H. Zhu

Universität Zürich, Zurich, Switzerland

C. Amsler ${ }^{64}$, P. Bärtschi, C. Botta, D. Brzhechko, M.F. Canelli, K. Cormier, A. De Wit, R. Del Burgo, J.K. Heikkilä, M. Huwiler, W. Jin, A. Jofrehei, B. Kilminster, S. Leontsinis, S.P. Liechti, A. Macchiolo, P. Meiring, V.M. Mikuni, U. Molinatti, I. Neutelings, A. Reimers, P. Robmann, S. Sanchez Cruz, K. Schweiger, Y. Takahashi

National Central University, Chung-Li, Taiwan

C. Adloff ${ }^{65}$, C.M. Kuo, W. Lin, A. Roy, T. Sarkar ${ }^{36}$, S.S. Yu

National Taiwan University (NTU), Taipei, Taiwan

L. Ceard, Y. Chao, K.F. Chen, P.H. Chen, W.-S. Hou, Y.y. Li, R.-S. Lu, E. Paganis, A. Psallidas, A. Steen, H.y. Wu, E. Yazgan, P.r. Yu

Chulalongkorn University, Faculty of Science, Department of Physics, Bangkok, Thailand

B. Asavapibhop, C. Asawatangtrakuldee, N. Srimanobhas

Çukurova University, Physics Department, Science and Art Faculty, Adana, Turkey

F. Boran, S. Damarseckin ${ }^{66}$, Z.S. Demiroglu, F. Dolek, I. Dumanoglu ${ }^{67}$, E. Eskut, Y. Guler ${ }^{68}$, E. Gurpinar Guler ${ }^{68}$, I. Hos $^{69}$, C. Isik, O. Kara, A. Kayis Topaksu, U. Kiminsu, G. Onengut, K. Ozdemir ${ }^{70}$, A. Polatoz, A.E. Simsek, B. Tali ${ }^{71}$, U.G. Tok, S. Turkcapar, I.S. Zorbakir, C. Zorbilmez

Middle East Technical University, Physics Department, Ankara, Turkey B. Isildak ${ }^{72}$, G. Karapinar ${ }^{73}$, K. Ocalan ${ }^{74}$, M. Yalvac ${ }^{75}$

Bogazici University, Istanbul, Turkey

B. Akgun, I.O. Atakisi, E. Gülmez, M. Kaya ${ }^{76}$, O. Kaya ${ }^{77}$, Ö. Özçelik, S. Tekten ${ }^{78}$, E.A. Yetkin ${ }^{79}$

Istanbul Technical University, Istanbul, Turkey

A. Cakir, K. Cankocak ${ }^{67}$, Y. Komurcu, S. Sen ${ }^{80}$

Istanbul University, Istanbul, Turkey

S. Cerci ${ }^{71}$, B. Kaynak, S. Ozkorucuklu, D. Sunar Cerci ${ }^{71}$

Institute for Scintillation Materials of National Academy of Science of Ukraine, Kharkov, Ukraine

B. Grynyov

National Scientific Center, Kharkov Institute of Physics and Technology, Kharkov, Ukraine

L. Levchuk 


\section{University of Bristol, Bristol, United Kingdom}

D. Anthony, E. Bhal, S. Bologna, J.J. Brooke, A. Bundock, E. Clement, D. Cussans, H. Flacher, J. Goldstein, G.P. Heath, H.F. Heath, L. Kreczko, B. Krikler, S. Paramesvaran, S. Seif El Nasr-Storey, V.J. Smith, N. Stylianou ${ }^{81}$, K. Walkingshaw Pass, R. White

\section{Rutherford Appleton Laboratory, Didcot, United Kingdom}

K.W. Bell, A. Belyaev ${ }^{82}$, C. Brew, R.M. Brown, D.J.A. Cockerill, C. Cooke, K.V. Ellis, K. Harder, S. Harper, M.l. Holmberg ${ }^{83}$, J. Linacre, K. Manolopoulos, D.M. Newbold, E. Olaiya, D. Petyt, T. Reis, T. Schuh, C.H. Shepherd-Themistocleous, I.R. Tomalin, T. Williams

\section{Imperial College, London, United Kingdom}

R. Bainbridge, P. Bloch, S. Bonomally, J. Borg, S. Breeze, O. Buchmuller, V. Cepaitis, G.S. Chahal ${ }^{84}$, D. Colling, P. Dauncey, G. Davies, M. Della Negra, S. Fayer, G. Fedi, G. Hall, M.H. Hassanshahi, G. Iles, J. Langford, L. Lyons, A.-M. Magnan, S. Malik, A. Martelli, D.G. Monk, J. Nash ${ }^{85}$, M. Pesaresi, D.M. Raymond, A. Richards, A. Rose, E. Scott, C. Seez, A. Shtipliyski, A. Tapper, K. Uchida, T. Virdee ${ }^{19}$, M. Vojinovic, N. Wardle, S.N. Webb, D. Winterbottom

\section{Brunel University, Uxbridge, United Kingdom}

K. Coldham, J.E. Cole, A. Khan, P. Kyberd, I.D. Reid, L. Teodorescu, S. Zahid

Baylor University, Waco, U.S.A.

S. Abdullin, A. Brinkerhoff, B. Caraway, J. Dittmann, K. Hatakeyama, A.R. Kanuganti, B. McMaster, N. Pastika, M. Saunders, S. Sawant, C. Sutantawibul, J. Wilson

Catholic University of America, Washington, DC, U.S.A.

R. Bartek, A. Dominguez, R. Uniyal, A.M. Vargas Hernandez

The University of Alabama, Tuscaloosa, U.S.A.

A. Buccilli, S.I. Cooper, D. Di Croce, S.V. Gleyzer, C. Henderson, C.U. Perez, P. Rumerio ${ }^{86}$, C. West

Boston University, Boston, U.S.A.

A. Akpinar, A. Albert, D. Arcaro, C. Cosby, Z. Demiragli, E. Fontanesi, D. Gastler, S. May, J. Rohlf, K. Salyer, D. Sperka, D. Spitzbart, I. Suarez, A. Tsatsos, S. Yuan, D. Zou

\section{Brown University, Providence, U.S.A.}

G. Benelli, B. Burkle, X. Coubez ${ }^{20}$, D. Cutts, M. Hadley, U. Heintz, J.M. Hogan ${ }^{87}$, G. Landsberg, K.T. Lau, M. Lukasik, J. Luo, M. Narain, S. Sagir ${ }^{88}$, E. Usai, W.Y. Wong, X. Yan, D. Yu, W. Zhang

University of California, Davis, Davis, U.S.A.

J. Bonilla, C. Brainerd, R. Breedon, M. Calderon De La Barca Sanchez, M. Chertok, J. Conway, P.T. Cox, R. Erbacher, G. Haza, F. Jensen, O. Kukral, R. Lander, M. Mulhearn, D. Pellett, B. Regnery, D. Taylor, Y. Yao, F. Zhang 
University of California, Los Angeles, U.S.A.

M. Bachtis, R. Cousins, A. Datta, D. Hamilton, J. Hauser, M. Ignatenko, M.A. Iqbal, T. Lam, W.A. Nash, S. Regnard, D. Saltzberg, B. Stone, V. Valuev

University of California, Riverside, Riverside, U.S.A.

K. Burt, Y. Chen, R. Clare, J.W. Gary, M. Gordon, G. Hanson, G. Karapostoli, O.R. Long, N. Manganelli, M. Olmedo Negrete, W. Si, S. Wimpenny, Y. Zhang

University of California, San Diego, La Jolla, U.S.A.

J.G. Branson, P. Chang, S. Cittolin, S. Cooperstein, N. Deelen, D. Diaz, J. Duarte, R. Gerosa, L. Giannini, D. Gilbert, J. Guiang, R. Kansal, V. Krutelyov, R. Lee, J. Letts, M. Masciovecchio, M. Pieri, B.V. Sathia Narayanan, V. Sharma, M. Tadel, A. Vartak, F. Würthwein, Y. Xiang, A. Yagil

University of California, Santa Barbara - Department of Physics, Santa Barbara, U.S.A.

N. Amin, C. Campagnari, M. Citron, A. Dorsett, V. Dutta, J. Incandela, M. Kilpatrick, J. Kim, B. Marsh, H. Mei, M. Oshiro, M. Quinnan, J. Richman, U. Sarica, F. Setti, J. Sheplock, D. Stuart, S. Wang

California Institute of Technology, Pasadena, U.S.A.

A. Bornheim, O. Cerri, I. Dutta, J.M. Lawhorn, N. Lu, J. Mao, H.B. Newman, T.Q. Nguyen, M. Spiropulu, J.R. Vlimant, C. Wang, S. Xie, Z. Zhang, R.Y. Zhu

Carnegie Mellon University, Pittsburgh, U.S.A.

J. Alison, S. An, M.B. Andrews, P. Bryant, T. Ferguson, A. Harilal, C. Liu, T. Mudholkar, M. Paulini, A. Sanchez, W. Terrill

University of Colorado Boulder, Boulder, U.S.A.

J.P. Cumalat, W.T. Ford, A. Hassani, E. MacDonald, R. Patel, A. Perloff, C. Savard, K. Stenson, K.A. Ulmer, S.R. Wagner

Cornell University, Ithaca, U.S.A.

J. Alexander, S. Bright-thonney, Y. Cheng, D.J. Cranshaw, S. Hogan, J. Monroy, J.R. Patterson, D. Quach, J. Reichert, M. Reid, A. Ryd, W. Sun, J. Thom, P. Wittich, R. Zou

Fermi National Accelerator Laboratory, Batavia, U.S.A.

M. Albrow, M. Alyari, G. Apollinari, A. Apresyan, A. Apyan, S. Banerjee, L.A.T. Bauerdick, D. Berry, J. Berryhill, P.C. Bhat, K. Burkett, J.N. Butler, A. Canepa, G.B. Cerati, H.W.K. Cheung, F. Chlebana, M. Cremonesi, K.F. Di Petrillo, V.D. Elvira, Y. Feng, J. Freeman, Z. Gecse, L. Gray, D. Green, S. Grünendahl, O. Gutsche, R.M. Harris, R. Heller, T.C. Herwig, J. Hirschauer, B. Jayatilaka, S. Jindariani, M. Johnson, U. Joshi, T. Klijnsma, B. Klima, K.H.M. Kwok, S. Lammel, D. Lincoln, R. Lipton, T. Liu, C. Madrid, K. Maeshima, C. Mantilla, D. Mason, P. McBride, P. Merkel, S. Mrenna, S. Nahn, J. Ngadiuba, V. O'Dell, V. Papadimitriou, K. Pedro, C. Pena ${ }^{56}$, O. Prokofyev, F. Ravera, A. Reinsvold Hall, L. Ristori, E. Sexton-Kennedy, N. Smith, A. Soha, W.J. Spalding, L. Spiegel, S. Stoynev, J. Strait, L. Taylor, S. Tkaczyk, N.V. Tran, L. Uplegger, E.W. Vaandering, H.A. Weber 
University of Florida, Gainesville, U.S.A.

D. Acosta, P. Avery, D. Bourilkov, L. Cadamuro, V. Cherepanov, F. Errico, R.D. Field,

D. Guerrero, B.M. Joshi, M. Kim, E. Koenig, J. Konigsberg, A. Korytov, K.H. Lo,

K. Matchev, N. Menendez, G. Mitselmakher, A. Muthirakalayil Madhu, N. Rawal,

D. Rosenzweig, S. Rosenzweig, J. Rotter, K. Shi, J. Sturdy, J. Wang, E. Yigitbasi, X. Zuo

Florida State University, Tallahassee, U.S.A.

T. Adams, A. Askew, R. Habibullah, V. Hagopian, K.F. Johnson, R. Khurana, T. Kolberg,

G. Martinez, H. Prosper, C. Schiber, O. Viazlo, R. Yohay, J. Zhang

Florida Institute of Technology, Melbourne, U.S.A.

M.M. Baarmand, S. Butalla, T. Elkafrawy ${ }^{89}$, M. Hohlmann, R. Kumar Verma, D. Noonan, M. Rahmani, F. Yumiceva

University of Illinois at Chicago (UIC), Chicago, U.S.A.

M.R. Adams, H. Becerril Gonzalez, R. Cavanaugh, X. Chen, S. Dittmer, O. Evdokimov, C.E. Gerber, D.A. Hangal, D.J. Hofman, A.H. Merrit, C. Mills, G. Oh, T. Roy, S. Rudrabhatla, M.B. Tonjes, N. Varelas, J. Viinikainen, X. Wang, Z. Wu, Z. Ye

The University of Iowa, Iowa City, U.S.A.

M. Alhusseini, K. Dilsiz ${ }^{90}$, R.P. Gandrajula, O.K. Köseyan, J.-P. Merlo, A. Mestvirishvili ${ }^{91}$, J. Nachtman, H. Ogul ${ }^{92}$, Y. Onel, A. Penzo, C. Snyder, E. Tiras ${ }^{93}$

Johns Hopkins University, Baltimore, U.S.A.

O. Amram, B. Blumenfeld, L. Corcodilos, J. Davis, M. Eminizer, A.V. Gritsan, S. Kyriacou, P. Maksimovic, J. Roskes, M. Swartz, T.Á. Vámi

The University of Kansas, Lawrence, U.S.A.

A. Abreu, J. Anguiano, C. Baldenegro Barrera, P. Baringer, A. Bean, A. Bylinkin, Z. Flowers, T. Isidori, S. Khalil, J. King, G. Krintiras, A. Kropivnitskaya, M. Lazarovits, C. Lindsey, J. Marquez, N. Minafra, M. Murray, M. Nickel, C. Rogan, C. Royon, R. Salvatico, S. Sanders, E. Schmitz, C. Smith, J.D. Tapia Takaki, Q. Wang, Z. Warner, J. Williams, G. Wilson

Kansas State University, Manhattan, U.S.A.

S. Duric, A. Ivanov, K. Kaadze, D. Kim, Y. Maravin, T. Mitchell, A. Modak, K. Nam

Lawrence Livermore National Laboratory, Livermore, U.S.A.

F. Rebassoo, D. Wright

University of Maryland, College Park, U.S.A.

E. Adams, A. Baden, O. Baron, A. Belloni, S.C. Eno, N.J. Hadley, S. Jabeen, R.G. Kellogg, T. Koeth, A.C. Mignerey, S. Nabili, C. Palmer, M. Seidel, A. Skuja, L. Wang, K. Wong

Massachusetts Institute of Technology, Cambridge, U.S.A.

D. Abercrombie, G. Andreassi, R. Bi, S. Brandt, W. Busza, I.A. Cali, Y. Chen,

M. D'Alfonso, J. Eysermans, C. Freer, G. Gomez Ceballos, M. Goncharov, P. Harris,

M. Hu, M. Klute, D. Kovalskyi, J. Krupa, Y.-J. Lee, C. Mironov, C. Paus, D. Rankin,

C. Roland, G. Roland, Z. Shi, G.S.F. Stephans, J. Wang, Z. Wang, B. Wyslouch 
University of Minnesota, Minneapolis, U.S.A.

R.M. Chatterjee, A. Evans, P. Hansen, J. Hiltbrand, Sh. Jain, M. Krohn, Y. Kubota, J. Mans, M. Revering, R. Rusack, R. Saradhy, N. Schroeder, N. Strobbe, M.A. Wadud

University of Nebraska-Lincoln, Lincoln, U.S.A.

K. Bloom, M. Bryson, S. Chauhan, D.R. Claes, C. Fangmeier, L. Finco, F. Golf, C. Joo, I. Kravchenko, M. Musich, I. Reed, J.E. Siado, G.R. Snow ${ }^{\dagger}$, W. Tabb, F. Yan, A.G. Zecchinelli

State University of New York at Buffalo, Buffalo, U.S.A.

G. Agarwal, H. Bandyopadhyay, L. Hay, I. Iashvili, A. Kharchilava, C. McLean, D. Nguyen, J. Pekkanen, S. Rappoccio, A. Williams

Northeastern University, Boston, U.S.A.

G. Alverson, E. Barberis, Y. Haddad, A. Hortiangtham, J. Li, G. Madigan, B. Marzocchi, D.M. Morse, V. Nguyen, T. Orimoto, A. Parker, L. Skinnari, A. Tishelman-Charny, T. Wamorkar, B. Wang, A. Wisecarver, D. Wood

Northwestern University, Evanston, U.S.A.

S. Bhattacharya, J. Bueghly, Z. Chen, A. Gilbert, T. Gunter, K.A. Hahn, Y. Liu, N. Odell, M.H. Schmitt, M. Velasco

University of Notre Dame, Notre Dame, U.S.A.

R. Band, R. Bucci, A. Das, N. Dev, R. Goldouzian, M. Hildreth, K. Hurtado Anampa, C. Jessop, K. Lannon, J. Lawrence, N. Loukas, D. Lutton, N. Marinelli, I. Mcalister, T. McCauley, C. Mcgrady, K. Mohrman, Y. Musienko ${ }^{49}$, R. Ruchti, P. Siddireddy, A. Townsend, M. Wayne, A. Wightman, M. Zarucki, L. Zygala

The Ohio State University, Columbus, U.S.A.

B. Bylsma, B. Cardwell, L.S. Durkin, B. Francis, C. Hill, M. Nunez Ornelas, K. Wei, B.L. Winer, B.R. Yates

Princeton University, Princeton, U.S.A.

F.M. Addesa, B. Bonham, P. Das, G. Dezoort, P. Elmer, A. Frankenthal, B. Greenberg, N. Haubrich, S. Higginbotham, A. Kalogeropoulos, G. Kopp, S. Kwan, D. Lange, D. Marlow, K. Mei, I. Ojalvo, J. Olsen, D. Stickland, C. Tully

University of Puerto Rico, Mayaguez, U.S.A.

S. Malik, S. Norberg

Purdue University, West Lafayette, U.S.A.

A.S. Bakshi, V.E. Barnes, R. Chawla, S. Das, L. Gutay, M. Jones, A.W. Jung, S. Karmarkar, D. Kondratyev, M. Liu, G. Negro, N. Neumeister, G. Paspalaki, C.C. Peng, S. Piperov, A. Purohit, J.F. Schulte, M. Stojanovic ${ }^{16}$, J. Thieman, F. Wang, R. Xiao, W. Xie

Purdue University Northwest, Hammond, U.S.A.

J. Dolen, N. Parashar 
Rice University, Houston, U.S.A.

A. Baty, M. Decaro, S. Dildick, K.M. Ecklund, S. Freed, P. Gardner, F.J.M. Geurts, A. Kumar, W. Li, B.P. Padley, R. Redjimi, W. Shi, A.G. Stahl Leiton, S. Yang, L. Zhang, Y. Zhang

University of Rochester, Rochester, U.S.A.

A. Bodek, P. de Barbaro, R. Demina, J.L. Dulemba, C. Fallon, T. Ferbel, M. Galanti, A. Garcia-Bellido, O. Hindrichs, A. Khukhunaishvili, E. Ranken, R. Taus

Rutgers, The State University of New Jersey, Piscataway, U.S.A.

B. Chiarito, J.P. Chou, A. Gandrakota, Y. Gershtein, E. Halkiadakis, A. Hart, M. Heindl, O. Karacheban ${ }^{23}$, I. Laflotte, A. Lath, R. Montalvo, K. Nash, M. Osherson, S. Salur, S. Schnetzer, S. Somalwar, R. Stone, S.A. Thayil, S. Thomas, H. Wang

University of Tennessee, Knoxville, U.S.A.

H. Acharya, A.G. Delannoy, S. Fiorendi, S. Spanier

Texas A\&M University, College Station, U.S.A.

O. Bouhali ${ }^{94}$, M. Dalchenko, A. Delgado, R. Eusebi, J. Gilmore, T. Huang, T. Kamon ${ }^{95}$, H. Kim, S. Luo, S. Malhotra, R. Mueller, D. Overton, D. Rathjens, A. Safonov

Texas Tech University, Lubbock, U.S.A.

N. Akchurin, J. Damgov, V. Hegde, S. Kunori, K. Lamichhane, S.W. Lee, T. Mengke, S. Muthumuni, T. Peltola, I. Volobouev, Z. Wang, A. Whitbeck

Vanderbilt University, Nashville, U.S.A.

E. Appelt, S. Greene, A. Gurrola, W. Johns, A. Melo, H. Ni, K. Padeken, F. Romeo, P. Sheldon, S. Tuo, J. Velkovska

University of Virginia, Charlottesville, U.S.A.

M.W. Arenton, B. Cox, G. Cummings, J. Hakala, R. Hirosky, M. Joyce, A. Ledovskoy, A. Li, C. Neu, B. Tannenwald, S. White, E. Wolfe

Wayne State University, Detroit, U.S.A.

N. Poudyal

University of Wisconsin - Madison, Madison, WI, U.S.A.

K. Black, T. Bose, C. Caillol, S. Dasu, I. De Bruyn, P. Everaerts, F. Fienga, C. Galloni, H. He, M. Herndon, A. Hervé, U. Hussain, A. Lanaro, A. Loeliger, R. Loveless, J. Madhusudanan Sreekala, A. Mallampalli, A. Mohammadi, D. Pinna, A. Savin, V. Shang, V. Sharma, W.H. Smith, D. Teague, S. Trembath-Reichert, W. Vetens

$\dagger$ : Deceased

1: Also at TU Wien, Wien, Austria

2: Also at Institute of Basic and Applied Sciences, Faculty of Engineering, Arab Academy for Science, Technology and Maritime Transport, Alexandria, Egypt, Alexandria, Egypt

3: Also at Université Libre de Bruxelles, Bruxelles, Belgium

4: Also at Universidade Estadual de Campinas, Campinas, Brazil 
5: Also at Federal University of Rio Grande do Sul, Porto Alegre, Brazil

6: Also at University of Chinese Academy of Sciences, Beijing, China

7: Also at Department of Physics, Tsinghua University, Beijing, China, Beijing, China

8: Also at UFMS, Nova Andradina, Brazil

9: Also at Nanjing Normal University Department of Physics, Nanjing, China

10: Now at The University of Iowa, Iowa City, U.S.A.

11: Also at Institute for Theoretical and Experimental Physics named by A.I. Alikhanov of NRC 'Kurchatov Institute', Moscow, Russia

12: Also at Joint Institute for Nuclear Research, Dubna, Russia

13: Also at Cairo University, Cairo, Egypt

14: Also at Suez University, Suez, Egypt

15: Now at British University in Egypt, Cairo, Egypt

16: Also at Purdue University, West Lafayette, U.S.A.

17: Also at Université de Haute Alsace, Mulhouse, France

18: Also at Erzincan Binali Yildirim University, Erzincan, Turkey

19: Also at CERN, European Organization for Nuclear Research, Geneva, Switzerland

20: Also at RWTH Aachen University, III. Physikalisches Institut A, Aachen, Germany

21: Also at University of Hamburg, Hamburg, Germany

22: Also at Department of Physics, Isfahan University of Technology, Isfahan, Iran, Isfahan, Iran

23: Also at Brandenburg University of Technology, Cottbus, Germany

24: Also at Forschungszentrum Jülich, Juelich, Germany

25: Also at Physics Department, Faculty of Science, Assiut University, Assiut, Egypt

26: Also at Karoly Robert Campus, MATE Institute of Technology, Gyongyos, Hungary

27: Also at Institute of Physics, University of Debrecen, Debrecen, Hungary, Debrecen, Hungary

28: Also at Institute of Nuclear Research ATOMKI, Debrecen, Hungary

29: Also at MTA-ELTE Lendület CMS Particle and Nuclear Physics Group, Eötvös Loránd University, Budapest, Hungary, Budapest, Hungary

30: Also at Wigner Research Centre for Physics, Budapest, Hungary

31: Also at IIT Bhubaneswar, Bhubaneswar, India, Bhubaneswar, India

32: Also at Institute of Physics, Bhubaneswar, India

33: Also at G.H.G. Khalsa College, Punjab, India

34: Also at Shoolini University, Solan, India

35: Also at University of Hyderabad, Hyderabad, India

36: Also at University of Visva-Bharati, Santiniketan, India

37: Also at Indian Institute of Technology (IIT), Mumbai, India

38: Also at Deutsches Elektronen-Synchrotron, Hamburg, Germany

39: Also at Sharif University of Technology, Tehran, Iran

40: Also at Department of Physics, University of Science and Technology of Mazandaran, Behshahr, Iran

41: Now at INFN Sezione di Bari ${ }^{a}$, Università di Bari ${ }^{b}$, Politecnico di Bari ${ }^{c}$, Bari, Italy

42: Also at Italian National Agency for New Technologies, Energy and Sustainable Economic Development, Bologna, Italy

43: Also at Centro Siciliano di Fisica Nucleare e di Struttura Della Materia, Catania, Italy

44: Also at Università di Napoli 'Federico II', NAPOLI, Italy

45: Also at Consiglio Nazionale delle Ricerche - Istituto Officina dei Materiali, PERUGIA, Italy

46: Also at Riga Technical University, Riga, Latvia, Riga, Latvia

47: Also at Consejo Nacional de Ciencia y Tecnología, Mexico City, Mexico

48: Also at IRFU, CEA, Université Paris-Saclay, Gif-sur-Yvette, France 
49: Also at Institute for Nuclear Research, Moscow, Russia

50: Now at National Research Nuclear University 'Moscow Engineering Physics Institute' (MEPhI), Moscow, Russia

51: Also at Institute of Nuclear Physics of the Uzbekistan Academy of Sciences, Tashkent, Uzbekistan

52: Also at St. Petersburg State Polytechnical University, St. Petersburg, Russia

53: Also at University of Florida, Gainesville, U.S.A.

54: Also at Imperial College, London, United Kingdom

55: Also at P.N. Lebedev Physical Institute, Moscow, Russia

56: Also at California Institute of Technology, Pasadena, U.S.A.

57: Also at Budker Institute of Nuclear Physics, Novosibirsk, Russia

58: Also at Faculty of Physics, University of Belgrade, Belgrade, Serbia

59: Also at Trincomalee Campus, Eastern University, Sri Lanka, Nilaveli, Sri Lanka

60: Also at INFN Sezione di Pavia ${ }^{a}$, Università di Pavia ${ }^{b}$, Pavia, Italy, Pavia, Italy

61: Also at National and Kapodistrian University of Athens, Athens, Greece

62: Also at Ecole Polytechnique Fédérale Lausanne, Lausanne, Switzerland

63: Also at Universität Zürich, Zurich, Switzerland

64: Also at Stefan Meyer Institute for Subatomic Physics, Vienna, Austria, Vienna, Austria

65: Also at Laboratoire d'Annecy-le-Vieux de Physique des Particules, IN2P3-CNRS, Annecyle-Vieux, France

66: Also at Şırnak University, Sirnak, Turkey

67: Also at Near East University, Research Center of Experimental Health Science, Nicosia, Turkey

68: Also at Konya Technical University, Konya, Turkey

69: Also at Istanbul University - Cerrahpasa, Faculty of Engineering, Istanbul, Turkey

70: Also at Piri Reis University, Istanbul, Turkey

71: Also at Adiyaman University, Adiyaman, Turkey

72: Also at Ozyegin University, Istanbul, Turkey

73: Also at Izmir Institute of Technology, Izmir, Turkey

74: Also at Necmettin Erbakan University, Konya, Turkey

75: Also at Bozok Universitetesi Rektörlügü, Yozgat, Turkey, Yozgat, Turkey

76: Also at Marmara University, Istanbul, Turkey

77: Also at Milli Savunma University, Istanbul, Turkey

78: Also at Kafkas University, Kars, Turkey

79: Also at Istanbul Bilgi University, Istanbul, Turkey

80: Also at Hacettepe University, Ankara, Turkey

81: Also at Vrije Universiteit Brussel, Brussel, Belgium

82: Also at School of Physics and Astronomy, University of Southampton, Southampton, United Kingdom

83: Also at Rutherford Appleton Laboratory, Didcot, United Kingdom

84: Also at IPPP Durham University, Durham, United Kingdom

85: Also at Monash University, Faculty of Science, Clayton, Australia

86: Also at Università di Torino, TORINO, Italy

87: Also at Bethel University, St. Paul, Minneapolis, U.S.A., St. Paul, U.S.A.

88: Also at Karamanoğlu Mehmetbey University, Karaman, Turkey

89: Also at Ain Shams University, Cairo, Egypt

90: Also at Bingol University, Bingol, Turkey

91: Also at Georgian Technical University, Tbilisi, Georgia 
92: Also at Sinop University, Sinop, Turkey

93: Also at Erciyes University, KAYSERI, Turkey

94: Also at Texas A\&M University at Qatar, Doha, Qatar

95: Also at Kyungpook National University, Daegu, Korea, Daegu, Korea 